\title{
On the Uniqueness for the Cauchy Problem for Elliptic Equations with Triple Characteristics
}

By

Shin-ichi FUJII*

\section{Contents}

$\S 1$. Introduction

$\S 2$. Factorization

$\S 3 . \quad$ Local Carleman Estimates

$\S$ 4. Proof of Proposition 1.1

$\S 5$. Pseudodifferential Operators

$\S 6$. Proof of Proposition 3.2

$\S 7$. Proofs of Lemmas in $\S 3$

$\S 8$. Invaricnce of the Assumptions in Theorem 1.1

$\S 9$. Proof of Theorem 1.1

References

\section{§1. Introduction}

In this paper we shall prove an uniqueness theorem for the Cauchy problem for certain elliptic differential operator $P(x, D)$ in a neighbourhood of the origin in $\boldsymbol{R}^{n}$ of order $m \geq 1$ with $C^{\infty}$-coefficients and the principal symbol $P_{m}(x, \xi)$ of the form

$$
P_{m}(x, \xi)=Q_{1}(x, \xi)^{2} Q_{2}(x, \xi)
$$

where $Q_{i}(i=1,2)$ is a homogeneous polynomial in $\xi$ of degree $m_{i}$ with $C^{\infty}$ coefficients such that

Communicated by S. Matsuura, February 13, 1991.

1991 Mathematics Subject Classifications: 35A07

* Research Institute for Mathematical Sciences, Kyoto University, Kyoto 606, Japan. 
if $m_{i} \geq 1$, for every $\xi^{\prime} \in \mathbb{R}^{n-1} \backslash(0)$ the zeros $\tau$ of $Q_{i}\left(0,\left(\tau, \xi^{\prime}\right)\right)$ are non-real and simple.

Our theorem is an extension of the Watanabe's theorem [6] in case of $C^{\infty}$ coefficients. Our main result is the following.

Theorem 1.1. Let $P(x, D)$ be a differential operator in an open neighborhood $\Omega$ of the origin in $\mathbb{R}^{n}$ of order $m \geq 1$ with $C^{\infty}$-coefficients and the symbol $P(x, \xi)$. Let $P(x, \xi)=P_{m}(x, \xi)+\cdots+P_{0}(x, \xi)$ with homogeneous polynomials $P_{j}(x, \xi)$ in $\xi$ of degree $j$. We assume the followings.

(i) The principal symbol $P_{m}$ of $P$ takes the form (1.1) with $Q_{i}$ as above.

(ii) If $P_{m}=\partial_{\xi_{1}} P_{m}=\partial_{\xi_{1}}^{2} P_{m}=0$ at $\left(0,\left(\tau_{0}, \eta_{0}\right)\right)$ with non-real $\tau_{0}$ and $\eta_{0} \in \mathbb{R}^{n-1} \backslash(0)$, there exists an open conic set $\Gamma$ in $\Omega \times\left(\mathbb{C}^{n} \backslash(0)\right)$ containing $\left(0,\left(\tau_{0}, \eta_{0}\right)\right)$ satisfying the following condition.

$$
\begin{aligned}
& \left(|\zeta|\left|\left(\partial_{\xi} P_{m}\right)(x, \zeta)\right|+\left|\left(\partial_{x} P_{m}\right)(x, \zeta)\right|\right)\left|P_{m-1}(x, \zeta)\right| \\
& \quad \leq C\left|P_{m}(x, \zeta)\right|^{2 / 3}\left(\left|P_{m}(x, \zeta)\right|^{1 / 3}|\zeta|^{m-1}+\left|\left(P_{m}+P_{m-1}\right)(x, \zeta)\right||\zeta|^{m / 3}\right. \\
& \left.\quad+(1+|\zeta|)^{(4 m / 3)-(3 / 2)}\right) \\
& \quad \text { for }(x, \zeta) \in \Gamma \text { with }\left(\partial_{\xi_{1}} P_{m}\right)(x, \zeta)=0 .
\end{aligned}
$$

Under the assumptions (i), (ii) there exists an open neighbourhood $\Omega^{\prime} \subset \Omega$ of the origin in $\mathbb{R}^{n}$ such that every $u \in C^{\infty}(\Omega)$ satisfying $P(x, D) u=0$ in $\Omega$ and $\left.u\right|_{x_{1} \leq 0}=0$ vanishes in $\Omega^{\prime}$.

Now, we give simple examples of differential operators $P(x, D)$ satisfying the assumptions of Theorem 1.1.

Example 1.1. Let $p(x, \xi)=\left(\xi_{1}-i \xi_{2}\right)^{2}\left(\xi_{1}-i \xi_{2}-a(x) \xi_{2}\right)$ and $q(x, \xi)=b(x) \xi_{2}^{2}$ where $a, b$ are $C^{\infty}$-functions in an open neighbourhood of the origin in $\mathbb{R}^{2}$. We assume that $a(0)=0$ and $|b(x)||d a(x)| \leq C|a(x)|$ near the origin. Then $P(x, D)=p(x, D)+q(x, D)$ satisfies the assumptions in Theorem 1.1 .

Indeed we have $\partial_{\xi_{1}} p=3\left(\xi_{1}-i \xi_{2}\right)\left(\xi_{1}-i \xi_{2}-\frac{2}{3} a(x) \xi_{2}\right)$. When $\xi_{1}-i \xi_{2}-$ $\frac{2}{3} a(x) \xi_{2}=0,(x, \xi) \in \mathbb{R}^{2} \times \mathbb{C}^{2}$ and when $\left|x^{\prime}\right|$ is small, we have that

$$
\begin{gathered}
\left(\left|\partial_{x} p\right|+\left|\partial_{\xi} p\right||\xi|\right)|q| \leq C(|d a||b|+|a|)|a|^{2}\left|\xi_{2}\right|^{5}, \\
|p||\xi|^{2} \geq \delta|a|^{3}\left|\xi_{2}\right|^{5} \text { for some } \delta>0 .
\end{gathered}
$$

Thus the inequality in the assumption (ii) in the theorem holds when $\xi_{1}=i \xi_{2}+$ $\frac{2}{3} a(x) \xi_{2},(x, \xi) \in \mathbb{R}^{2} \times \mathbb{C}^{2}$ and $\left|x^{\prime}\right|$ is small. This means that the assumption 
in the theorem holds for $P(x, D)$.

Example 1.2. Let $p(x, \xi)=\left(\xi_{1}^{2}+\cdots+\xi_{n}^{2}\right)^{2}\left(\xi_{1}^{2}+\cdots+\xi_{n}^{2}+a\left(x^{\prime}, \xi^{\prime}\right)\right)$ where $a\left(x^{\prime}, \xi^{\prime}\right)=\left|x^{\prime}\right|^{2 k}\left(\xi_{2}^{2}+\cdots+\xi_{n}^{2}\right)+x_{2}^{2 k_{2}} \xi_{2}^{2}+\cdots+x_{n}^{2 k_{n}} \xi_{n}^{2}$ with $k, k_{j} \in N, k_{j}>k>0$. Here we use a notation that $x^{\prime}=\left(x_{2}, \cdots, x_{n}\right)$. Let $q(x, \xi)=c_{1}\left(x, \xi^{\prime}\right) \xi_{1}+c_{0}\left(x, \xi^{\prime}\right)$ where $c_{1}, c_{0}$ are respectively homogeneous polynomials in $\xi^{\prime}$ of degree 4,5 with $C^{\infty}$-coefficients in an open neighbourhood of the origin in $\boldsymbol{R}^{n}$. We assume that $\left|c_{1}(x, \zeta)\right||\zeta|+\left|c_{0}(x, \zeta)\right| \leq C\left|x^{\prime}\right||\zeta|^{5}$ for small $|x|$ and $\zeta \in C^{n-1}$. Then $P(x, D)$ $=p(x, D)+q(x, D)$ satisfies the assumptions in Theorem 1.1 .

Indeed we have $\partial_{\xi_{1}} p=6\left(\xi_{1}^{2}+\cdots+\xi_{n}^{2}\right) \xi_{1}\left(\xi_{1}^{2}+\cdots+\xi_{n}^{2}+\frac{2}{3} a\right)$. Assume that $\xi_{1}^{2}+\cdots+\xi_{n}^{2}+\frac{2}{3} a=0,(x, \xi) \in \boldsymbol{R}^{n} \times \boldsymbol{C}^{n}$ with $\left|\operatorname{Im} \xi^{\prime}\right|<\frac{1}{2}\left|\operatorname{Re} \xi^{\prime}\right|$ and $\left|x^{\prime}\right|$ is small. Then $\left|x^{\prime}\right|\left|\partial_{x_{j}} a\right| \leq C|a|$ and $\left|\partial_{\xi_{j}} a\right||\xi| \leq C|a|$, because $|a| \geq$ $\delta\left|\xi^{\prime}\right|^{2}\left|x^{\prime}\right|^{2 k}$ for some $\delta>0$. Since $\left|\partial_{x} p\right|+\left|\partial_{\xi} p\right||\xi| \leq C\left(\left|\partial_{x^{\prime}} a\right|+\left|\partial_{\xi^{\prime}} a\right||\xi|\right)$ $|a|^{2}$ and $|p||\xi|^{5}=\frac{4}{27}|a|^{2}|\xi|^{5}$, the inequality in the assumption (ii) in the theorem holds. This means that the assumption in the theorem holds for $P(x, D)$.

The main part of proof of Theorem 1.1 is to derive Carleman estimate for some third order elliptic operators in the following proposition.

Proposition 1.1. Let $P=p(x, D)+q(x, D)$ be a pseudo-differential operator on $\boldsymbol{R}^{n}$ with $p(x, \xi), q(x, \xi)$ of the form

$$
\begin{aligned}
& p(x, \xi)=\left(\xi_{1}-\lambda\left(x, \xi^{\prime}\right)\right)^{2}\left(\xi_{1}-\lambda\left(x, \xi^{\prime}\right)+c\left(x, \xi^{\prime}\right)\right), \lambda, c \in S_{1,0}^{1}\left(\boldsymbol{R}^{n} \times \boldsymbol{R}^{n-1}\right) ; \\
& q(x, \xi)=\sum_{j=0}^{2} a_{j}\left(x, \xi^{\prime}\right) \xi_{1}^{j}, \quad a_{j}\left(x, \xi^{\prime}\right) \in S_{1,0}^{2-j}\left(\boldsymbol{R}^{n} \times \boldsymbol{R}^{n-1}\right)
\end{aligned}
$$

with $C|\operatorname{Im} \lambda| \geq\left\langle\xi^{\prime}\right\rangle,\left|\operatorname{Im} \lambda\left(x, \xi^{\prime}\right)\right| \geq 2\left|c\left(x, \xi^{\prime}\right)\right|$. Assume that

$$
\left(\left|\partial_{x} p\right|+\left|\partial_{\xi^{\prime}} p\right|\left|\xi^{\prime}\right|\right)|q| \leq C|p|^{2 / 3}\left(|p|^{1 / 3}\left|\xi^{\prime}\right|^{2}+|p+q|\left|\xi^{\prime}\right|+\left|\xi^{\prime}\right|^{5 / 2}+1\right)
$$

for all $(x, \xi) \in \boldsymbol{R}^{n} \times\left(\boldsymbol{C} \times \boldsymbol{R}^{n-1}\right)$ with $\partial_{\xi_{1}} p(x, \xi)=0$. Then there exist constants $\tau_{0}>0$ and $C_{0}>0$ such that if $\tau T^{2}>\tau_{0}$ and $T^{-1}>\tau_{0}$,

$$
T^{-1 / 2} \sum_{1 \leq|\alpha|+; \beta \mid \leq 2}\left\|E_{(|\alpha|-|\beta|) / 2} P_{(\beta)}^{(\alpha)} u\right\|_{T}^{(\tau)}+\|\| u\left\|_{T, 6}^{(\tau)} \leq C_{0}\right\| P u \|_{T}^{(\tau)}, u \in \mathcal{S}_{T / 2}\left(\boldsymbol{R}^{n}\right) .
$$

Here, by definition

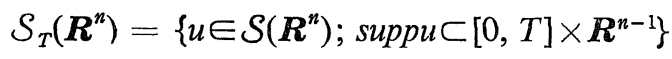

where $\mathcal{S}\left(\boldsymbol{R}^{n}\right)$ denotes the space of all rapidly decreasing $C^{\infty}$-functions on $\boldsymbol{R}^{n}$; 


$$
\begin{gathered}
\|u\|_{T}^{(\tau)}=\left\|e^{\tau\left(x_{1}-T\right)^{2} / 2} u\right\|_{L^{2}\left(\boldsymbol{R}^{n}\right)} \text { for } u \in \mathcal{S}\left(\mathbb{R}^{n}\right) ; \\
\|u\|\left\|_{T, s}^{(\tau)}=\sum_{\substack{i / 2+j \leq s / 2 \\
i, j \in \boldsymbol{Z}_{+}}} \tau^{3 / 2-i / 2-j} T^{-i / 2-j}\right\| E_{\dot{z} / 2} D_{1}^{j} u \|_{T}^{(\tau)} \text { for } u \in \mathcal{S}\left(\mathbb{R}^{n}\right), \\
s=0,1, \cdots, 6, T>0,
\end{gathered}
$$

where $\boldsymbol{Z}_{+}$denotes the set of all non-negative integers and

$$
\begin{aligned}
D_{k} & =\frac{1}{i} \partial_{x_{k}}, \quad E_{s}=\left\langle D^{\prime}\right\rangle^{s} ; \\
P_{(\beta)}^{(\alpha)} & =\left[\partial_{\xi}^{\alpha} \partial_{x}^{\beta}(p+q)\right](x, D) .
\end{aligned}
$$

The assumption (ii) in Theorem 1.1 is a translation of that of the above proposition. The assumption in the proposition ensures a factorization of $P$ in the proposition into first order operators being differential operators in $x_{1}$ and pseudodifferential operators of Beals-Fefferman's class in $x^{\prime}$.

When $c\left(x, \xi^{\prime}\right) \equiv 0$ in the above proposition, our assumption on $P$ makes no condition on $q(x, \xi)$. Carleman estimates for elliptic pseudo-differential operators with smooth characteristics of arbitrary high multiplicity were studied by Watanabe-Zuily [7]. But our result is stronger than theirs in our case.

This paper is organized as follows. We devote ourselves to prove Proposition 1.1 from $\S 2$ to $\S 7$. Theorem 1.1 is proved as a corollary of the proposition in the next two sections. In $\$ 2$ we carry out local factorization of the operator $P$ in Proposition 1.1 modulo negligible terms. In $\S 3$ we derive local Carleman estimates for factorized operators. In $\S 4$ we prove Proposition 1.1 by patching local Carleman estimates which follow from the results in $\$ 2$ and $\S 3$. Several facts on pseudo-differential operators used to prove Proposition 1.1 are collected in $\S 5$. In $\S 6$ we prove Carleman estimates for first order factors which are essential in the argument in $\$ 4$. In $\$ 7$ we prove lemmas in $\S 3$ on symbolic calculus. In $\S 8$ we prove the invariance of the assumptions in Theorem 1.1 under changes of variables such as $y_{1}=x_{1}-\varphi\left(x^{\prime}\right), y_{j}=x_{j}(j \geq 2)$ where $\varphi \in C^{\infty}$ with $\varphi(0)=0, d \varphi(0)=0$. In $\S 9$ we prove Theorem 1.1 using the result in $\$ 8$, Proposition 1.1, and theorems of Calderón [2], Mizohata [5], and Hörmander [4].

\section{Acknowledgement}

I am grateful to Prof. S. Matsuura, Prof. N. Iwasaki, and Prof. K. Nishiwada for helpful suggestions and comments, and encouragement, and I wish to thank the referee for careful reading of my manuscript and helpful advice. 


\section{§2. Factorization}

Let $\lambda, c \in S_{1,0}^{1}\left(\boldsymbol{R}^{n} \times \boldsymbol{R}^{n-1}\right)$ satisfying

$$
\begin{gathered}
C\left|\operatorname{Im} \lambda\left(x, \xi^{\prime}\right)\right| \geq\left\langle\xi^{\prime}\right\rangle, \\
2\left|c\left(x, \xi^{\prime}\right)\right| \leq\left|\operatorname{Im} \lambda\left(x, \xi^{\prime}\right)\right| .
\end{gathered}
$$

Let

$$
\begin{gathered}
p(x, \xi)=\left(\xi_{1}-\lambda\left(x, \xi^{\prime}\right)\right)^{2}\left(\xi_{1}-\lambda\left(x, \xi^{\prime}\right)+c\left(x, \xi^{\prime}\right)\right), \\
q(x, \xi)=\sum_{j=0}^{2} a_{j}\left(x, \xi^{\prime}\right)\left(\xi_{1}-\lambda\left(x, \xi^{\prime}\right)\right)^{j} \quad \text { with } \quad a_{j} \in S_{1,0}^{2-j}\left(\boldsymbol{R}^{n} \times \boldsymbol{R}^{n-1}\right),
\end{gathered}
$$

and set

$$
P=p+q
$$

We have

$$
\partial_{\xi_{1}} p(x, \xi)=3\left(\xi_{1}-\lambda_{1}\left(x, \xi^{\prime}\right)\right)\left(\xi_{1}-\lambda_{2}\left(x, \xi^{\prime}\right)\right)
$$

with

$$
\lambda_{1}=\lambda, \lambda_{2}=\lambda-\frac{2}{3} c
$$

Then,

$$
p(x, \xi)=\left(\xi_{1}-\lambda_{2}\left(x, \xi^{\prime}\right)\right)^{2}\left(\xi_{1}-\lambda_{2}\left(x, \xi^{\prime}\right)-c\left(x, \xi^{\prime}\right)\right)+\frac{4}{27} c\left(x, \xi^{\prime}\right)^{3} .
$$

Since $\lambda_{1}-\lambda_{2}=\frac{2}{3} c, q$ can be expressed as

$$
q(x, \xi)=\sum_{j=0}^{2} b_{l j}\left(x, \xi^{\prime}\right)\left(\xi_{1}-\lambda_{l}\left(x, \xi^{\prime}\right)\right)^{j}, \quad l=1,2
$$

with $b_{1 j}=a_{j}, b_{2 j} \in S_{1,0}^{2-j}\left(\boldsymbol{R}^{n} \times \boldsymbol{R}^{n-1}\right)$ satisfying

$$
b_{2 j}-b_{1 j}=c d_{j} \text { for some } d_{j} \in S_{1,0}^{1-j}\left(\boldsymbol{R}^{n} \times \boldsymbol{R}^{n-1}\right) .
$$

Setting

$$
g_{1}=b_{10}, \quad g_{2}=\frac{4}{27} c^{3}+b_{20}, \quad c_{l}=(-1)^{l} c
$$

for $l=1,2$, and

$$
p_{l}(x, \xi)=\left(\xi_{1}-\lambda_{l}\left(x, \xi^{\prime}\right)\right)^{2}\left(\xi_{1}-\lambda_{l}\left(x, \xi^{\prime}\right)-c_{l}\left(x, \xi^{\prime}\right)\right)+g_{l}\left(x, \xi^{\prime}\right)
$$

we have

$$
P(x, \xi)=p_{l}(x, \xi)+\sum_{j=1}^{2} b_{l j}\left(x, \xi^{\prime}\right)\left(\xi_{1}-\lambda_{l}\left(x, \xi^{\prime}\right)\right)^{j}
$$


Now we deduce the estimates of derivatives of $g_{l}$ from the assumption of Proposition 1.1.

Lemma 2.1. Assume that

$\left(\left|\partial_{x} p\right|+\left|\partial_{\xi^{\prime}} p\right|\left|\xi^{\prime}\right|\right)|q| \leq C|p|^{2 / 3}\left(|p|^{1 / 3}\left|\xi^{\prime}\right|^{2}+|P|\left|\xi^{\prime}\right|+\left|\xi^{\prime}\right|^{5 / 2}+1\right)$

when $\partial_{\xi_{1}} p(x, \xi)=0, \quad(x, \xi) \in \mathbb{R}^{n} \times\left(\mathbb{C}_{\xi_{1}} \times \mathbb{R}_{\xi^{\prime}}^{n-1}\right)$.

Choose $\chi \in C^{\infty}(\mathbb{R})$ satisfying $\chi(t)=0$ when $t \leq \frac{1}{2}$ and $\chi(t)=1$ when $t \geq 1$. Define $\Phi_{l} \in C^{\infty}\left(\mathbb{R}^{n} \times \mathbb{R}^{n-1}\right)$ by

$$
\begin{aligned}
\Phi_{l}\left(x, \xi^{\prime}\right)^{-1}= & \frac{1}{\left\langle g_{l}\left(x, \xi^{\prime}\right)\right\rangle^{1 / 3}} \chi\left(\left\langle g_{l}\left(x, \xi^{\prime}\right)\right\rangle\left\langle\xi^{\prime}\right\rangle^{-3 / 2}\right) \\
& +\frac{1}{\left\langle\xi^{\prime}\right\rangle^{1 / 2}}(1-\chi)\left(2^{-1}\left\langle g_{l}\left(x, \xi^{\prime}\right)\right\rangle\left\langle\xi^{\prime}\right\rangle^{-3 / 2}\right)
\end{aligned}
$$

where $\langle z\rangle=(1+|z|)^{1 / 2}$ for $z \in \mathbb{C}$. We also define $\varphi_{l} \in C^{\infty}\left(\mathbb{R}^{n} \times \mathbb{R}^{n-1}\right)$ by

$$
\varphi_{l}\left(x, \xi^{\prime}\right)=\left\langle\xi^{\prime}\right\rangle^{-1} \Phi_{l}\left(x, \xi^{\prime}\right)
$$

Then, we have

$$
\left|\partial_{x}^{\alpha} \partial_{\xi^{\prime}}^{\beta} g_{l}\left(x, \xi^{\prime}\right)\right| \leq C_{\alpha \beta} \Phi_{l}^{3-|\beta|}\left(x, \xi^{\prime}\right) \varphi_{l}^{-|\alpha|}\left(x, \xi^{\prime}\right) \text { for any } \alpha, \beta \text {. }
$$

Proof. From (2.6), $\partial_{\xi_{1}} p=0$ means that $\xi_{1}=\lambda_{1}\left(x, \xi^{\prime}\right)$ or $\xi_{1}=\lambda_{2}\left(x, \xi^{\prime}\right)$. If $\xi_{1}=\lambda_{2}\left(x, \xi^{\prime}\right)$,

$$
\begin{gathered}
\partial_{x_{j}} p(x, \xi)=\frac{4}{9}\left(\partial_{x_{j}} c\right)\left(x, \xi^{\prime}\right) c\left(x, \xi^{\prime}\right)^{2}, \\
\partial_{\xi_{j}} p(x, \xi)=\frac{4}{9}\left(\partial_{\xi_{j}} c\right)\left(x, \xi^{\prime}\right) c\left(x, \xi^{\prime}\right)^{2}, \\
p(x, \xi)=\frac{4}{27} c(x, \xi)^{3}, \quad q(x, \xi)=b_{20}(x, \xi), \\
P(x, \xi)=g_{2}\left(x, \xi^{\prime}\right) .
\end{gathered}
$$

So from (2.13),

$$
\left(\sum_{j=1}^{n}\left|\partial_{x_{j}} c\right|+\sum_{j=2}^{n}\left|\partial_{\xi_{j}} c\right|\left|\xi^{\prime}\right|\right)\left|b_{20}\right| \leq C\left(|c|\left|\xi^{\prime}\right|^{2}+\left|g_{2}\right|\left|\xi^{\prime}\right|+\left|\xi^{\prime}\right|^{5 / 2}+1\right) \text {. }
$$

Now we shall show (2.16) for $l=1,2$. First we have

$$
\begin{aligned}
\partial_{x}^{\alpha} \partial_{\xi^{\prime}}^{\beta} g_{2}\left(x, \xi^{\prime}\right)= & \frac{4}{27} \sum_{\substack{\sum_{i=1}^{3} \alpha^{(i)}=\alpha \\
\sum_{i=1}^{3} \beta^{(i)}=\beta}} \frac{\alpha ! \beta !}{\prod_{i=1}^{3}\left(\alpha^{(i)} ! \beta^{(i)} !\right)} \prod_{i=1}^{3} \partial_{x}^{\alpha(i)} \partial_{\xi^{\prime}}^{\beta(i)} c\left(x, \xi^{\prime}\right) \\
& +\partial_{x}^{\alpha} \partial_{\xi^{\prime}}^{\beta} b_{20}\left(x, \xi^{\prime}\right) .
\end{aligned}
$$


We note that

$$
C^{-1}\left\langle\xi^{\prime}\right\rangle^{1 / 2} \leq \Phi_{l} \leq C\left\langle\xi^{\prime}\right\rangle, \quad C^{-1}\left\langle\xi^{\prime}\right\rangle^{-1 / 2} \leq \varphi_{l} \leq C \text { for some } C .
$$

To estimate the second term on the right hand side of (2.18) we show that for each $l$

$$
\begin{gathered}
\left|\partial_{x}^{\alpha} \partial_{\xi^{\prime}}^{\beta} a\left(x, \xi^{\prime}\right)\right| \leq C_{\alpha \beta} \Phi_{l}^{3-|\beta|}\left(x, \xi^{\prime}\right) \varphi_{l}^{-|\alpha|}\left(x, \xi^{\prime}\right) \\
\text { if } a \in S_{1,0}^{2}\left(\boldsymbol{R}^{n} \times \boldsymbol{R}^{n-1}\right) \text { and }|\alpha|+|\beta|>0 .
\end{gathered}
$$

Indeed, from (2.19)

$$
\begin{aligned}
\left\langle\xi^{\prime}\right\rangle^{2} & =\left(\Phi_{l}^{-3}\left(x, \xi^{\prime}\right)\left\langle\xi^{\prime}\right\rangle^{2}\right) \Phi_{l}^{3}\left(x, \xi^{\prime}\right) \\
& \leq C\left(\Phi_{l}^{-1}\left(x, \xi^{\prime}\right)\left\langle\xi^{\prime}\right\rangle\right) \Phi_{l}^{3}\left(x, \xi^{\prime}\right),
\end{aligned}
$$

and from (2.15)

$$
\left(\Phi_{l}^{-1}\left(x, \xi^{\prime}\right)\left\langle\xi^{\prime}\right\rangle\right)^{|\alpha|+|\beta|}\left\langle\xi^{\prime}\right\rangle^{-|\beta|}=\Phi_{l}^{-|\beta|}\left(x, \xi^{\prime}\right) \varphi_{l}^{-|\alpha|}\left(x, \xi^{\prime}\right) .
$$

Thus, if $|\alpha|+|\beta| \geq 1$, using (2.19), (2.21) we get

$$
\begin{aligned}
\left|\partial_{x}^{\alpha} \partial_{\xi^{\prime}}^{\beta} a\left(x, \xi^{\prime}\right)\right| & \leq C_{\alpha \beta}\left\langle\xi^{\prime}\right\rangle^{2-|\beta|} \\
& \leq C_{\alpha \beta}^{\prime}\left(\Phi_{l}^{-1}\left(x, \xi^{\prime}\right)\left\langle\xi^{\prime}\right\rangle\right) \Phi_{l}^{3}\left(x, \xi^{\prime}\right)\left\langle\xi^{\prime}\right\rangle^{-|\beta|} \\
& \leq C_{\alpha \beta}^{\prime \prime}\left(\Phi_{l}^{-1}\left(x, \xi^{\prime}\right)\left\langle\xi^{\prime}\right\rangle\right)^{|\alpha|+|\beta|} \Phi_{l}^{3}\left(x, \xi^{\prime}\right)\left\langle\xi^{\prime}\right\rangle^{-|\beta|} .
\end{aligned}
$$

This inequality and (2.22) mean (2.20). From (2.18) and (2.20), in order to show that (2.15) holds when $|\alpha|+|\beta|>0$, it suffices to show that

$$
\begin{gathered}
\left|\prod_{i=1}^{3} \partial_{x}^{\alpha(i)} \partial_{\xi^{\prime}}^{\beta(i)} c\left(x, \xi^{\prime}\right)\right| \leq C_{\alpha \beta} \Phi_{2}^{3-|\beta|}\left(x, \xi^{\prime}\right) \varphi_{2}^{-|\alpha|}\left(x, \xi^{\prime}\right) \\
\text { when }|\alpha|+|\beta|>0, \sum_{i=1}^{3} \alpha^{(i)}=\alpha, \sum_{i=1}^{3} \beta^{(i)}=\beta
\end{gathered}
$$

Set

$$
\begin{aligned}
& A_{1}=\left\{\left(x, \xi^{\prime}\right) \in \boldsymbol{R}^{n} \times \boldsymbol{R}^{n-1} ; \Phi_{l}\left(x, \xi^{\prime}\right) \leq\left|c\left(x, \xi^{\prime}\right)\right|\right\} \\
& A_{2}=\left\{\left(x, \xi^{\prime}\right) \in \boldsymbol{R}^{n} \times \boldsymbol{R}^{n-1} ; \Phi_{l}\left(x, \xi^{\prime}\right) \geq\left|c\left(x, \xi^{\prime}\right)\right|\right\}
\end{aligned}
$$

Case 1. Assume $\left(x, \xi^{\prime}\right) \in A_{1}$. We devide our argument into two subcases:

$$
\begin{array}{ll}
\left|\alpha^{(i)}\right|+\left|\beta^{(i)}\right| \leq 1 & \text { for any } i, \\
\left|\alpha^{(i)}\right|+\left|\beta^{(i)}\right| \geq 2 & \text { for some } i .
\end{array}
$$

First we assume (2.24). Put $J=\left\{i ;\left|\alpha^{(i)}\right|+\left|\beta^{(i)}\right| \neq 0\right\}$ and choose $i_{0} \in J$. Then,

$$
\begin{gathered}
\prod_{i=1}^{3} \partial_{x}^{\alpha(i)} \partial_{\xi^{\prime}}^{\beta(i)} c\left(x, \xi^{\prime}\right)=\frac{27}{4} g_{2}\left(x, \xi^{\prime}\right) \prod_{i \in J} \frac{\partial_{x}^{\alpha(i)} \partial_{\xi^{\prime}}^{\beta(i)} c\left(x, \xi^{\prime}\right)}{c\left(x, \xi^{\prime}\right)} \\
\quad-\frac{27}{4} b_{20}\left(x, \xi^{\prime}\right) \prod_{i \in J} \frac{\partial_{x}^{\alpha(i)} \partial_{\xi^{\prime}}^{\beta(i)} c\left(x, \xi^{\prime}\right)}{c\left(x, \xi^{\prime}\right)}=I+I I .
\end{gathered}
$$


Since $\sharp(J)=|\alpha|+|\beta|$, and since $\left(x, \xi^{\prime}\right) \in A_{1}$,

$$
|I| \leq C\left(\Phi_{2}\left(x, \xi^{\prime}\right)^{-1}\left\langle\xi^{\prime}\right\rangle\right)^{|\alpha|+|\beta|}\left\langle\xi^{\prime}\right\rangle^{-|\beta|} \Phi_{2}^{3}\left(x, \xi^{\prime}\right) .
$$

On the other hand, from (2.17) and the same reason as above,

$$
\begin{aligned}
|I I| \leq & C_{1}\left\langle\xi^{\prime}\right\rangle-\left.\left|\beta^{\left(i_{0}\right)}\left(\left|c\left(x, \xi^{\prime}\right)\right|\left|\xi^{\prime}\right|^{2}+\left|\xi^{\prime}\right|\left|g_{2}\left(x, \xi^{\prime}\right)\right|+\left|\xi^{\prime}\right|^{5 / 2}+1\right)\right| c\left(x, \xi^{\prime}\right)\right|^{-1} \\
& \times \prod_{i \in J \backslash\left\{i_{0}\right\}} \frac{\left|\partial_{x}^{\alpha(i)} \partial_{\xi^{\prime}}^{\beta^{(i)}} c\left(x, \xi^{\prime}\right)\right|}{\left|c\left(x, \xi^{\prime}\right)\right|} \\
\leq & C_{2}\left\{\left(\frac{\left\langle\xi^{\prime}\right\rangle}{\left|c\left(x, \xi^{\prime}\right)\right|}\right)^{\mid(J)-1}\left\langle\xi^{\prime}\right\rangle^{2-|\beta|}\right. \\
& \left.+\left(\frac{\left\langle\xi^{\prime}\right\rangle}{\left|c\left(x, \xi^{\prime}\right)\right|}\right)^{\mid(J)}\left\langle\xi^{\prime}\right\rangle^{-|\beta|}\left(\left|g_{2}\left(x, \xi^{\prime}\right)\right|+\left\langle\xi^{\prime}\right\rangle^{3 / 2}\right)\right\} \\
\leq & C_{3}\left\{\Phi_{2}^{-1}\left(x, \xi^{\prime}\right)\left\langle\xi^{\prime}\right\rangle\right)^{|\alpha|+|\beta|-1}\left\langle\xi^{\prime}\right\rangle^{2-|\beta|} \\
& \left.+\left(\Phi_{2}^{-1}\left(x, \xi^{\prime}\right)\left\langle\xi^{\prime}\right\rangle\right)^{|\alpha|+|\beta|}\left\langle\xi^{\prime}\right\rangle^{-|\beta|} \Phi_{2}^{3}\left(x, \xi^{\prime}\right)\right\}
\end{aligned}
$$

Applying (2.21) to the first term in the last expression we get

$$
|I I| \leq C_{4}\left(\Phi_{2}^{-1}\left(x, \xi^{\prime}\right)\left\langle\xi^{\prime}\right\rangle\right)^{|\alpha|+|\beta|}\left\langle\xi^{\prime}\right\rangle^{-|\beta|} \Phi_{2}^{3}\left(x, \xi^{\prime}\right) .
$$

(2.27), (2.28), and (2.22) mean (2.23). Next we assume (2.25). We have with the set $J$ as above,

$$
\begin{aligned}
& \prod_{i=1}^{3}\left|\partial_{x}^{\alpha(i)} \partial_{\xi^{\prime}}^{\beta(i)} c\left(x, \xi^{\prime}\right)\right| \leq C_{1}\left(\frac{\left\langle\xi^{\prime}\right\rangle}{\left|c\left(x, \xi^{\prime}\right)\right|}\right)^{(j(J)}\left\langle\xi^{\prime}\right\rangle^{-|\beta|}\left|c\left(x, \xi^{\prime}\right)\right|^{3} \\
& \quad \leq C_{2}\left(\frac{\left\langle\xi^{\prime}\right\rangle}{\left|c\left(x, \xi^{\prime}\right)\right|}{ }^{(J)}\left\langle\xi^{\prime}\right\rangle^{-|\beta|}\left(\left|g_{2}\left(x, \xi^{\prime}\right)\right|+\left|b_{20}\left(x, \xi^{\prime}\right)\right|\right)\right. \\
& \quad \leq C_{3}\left(\Phi_{2}^{-1}\left(x, \xi^{\prime}\right)\left\langle\xi^{\prime}\right\rangle\right)^{|\alpha|+|\beta|-1}\left\langle\xi^{\prime}\right\rangle^{-|\beta|}\left(\Phi_{2}^{3}\left(x, \xi^{\prime}\right)+\left\langle\xi^{\prime}\right\rangle^{2}\right),
\end{aligned}
$$

because $\sharp(J) \leq|\alpha|+|\beta|-1$. From (2.19), (2.21), and (2.22) we obtain (2.23).

Case 2. Assume that $(x, \xi) \in A_{2}$, and define the set $J$ as in Case 1. Noting that $\sharp(J) \leq \min \{3,|\alpha|+|\beta|\}$ we have

$$
\begin{aligned}
& \prod_{i=1}^{3}\left|\partial_{x}^{\alpha(i)} \partial_{\xi^{\prime}}^{\beta(i)} c\left(x, \xi^{\prime}\right)\right| \leq C\left\langle\xi^{\prime}\right\rangle^{\sharp(J)-|\beta|}\left|c\left(x, \xi^{\prime}\right)\right|^{3-\xi(J)} \\
& \leq C\left\langle\xi^{\prime}\right\rangle^{\sharp(J)-|\beta|} \Phi_{2}\left(x, \xi^{\prime}\right)^{3-\xi(J)} \\
& \leq C^{\prime}\left(\frac{\left\langle\xi^{\prime}\right\rangle}{\Phi_{2}\left(x, \xi^{\prime}\right)}\right)^{|\alpha|+|\beta|}\left\langle\xi^{\prime}\right\rangle^{-|\beta|} \Phi_{2}\left(x, \xi^{\prime}\right) \text {. }
\end{aligned}
$$

Thus (2.22) means (2.23).

Q.E.D.

Corollary 2.1. $\Phi_{l}$ and $\varphi_{l}$ satisfy the estimates

$$
\left|\partial_{x}^{\alpha} \partial_{\xi^{\prime}}^{\beta} \Phi_{l}\left(x, \xi^{\prime}\right)\right| \leq C_{\alpha \beta} \Phi_{l}^{1-|\beta|} \varphi_{l}^{-|\alpha|}\left(x, \xi^{\prime}\right),
$$




$$
\left|\partial_{x}^{\alpha} \partial_{\xi^{\prime}}^{\beta} \varphi_{l}\left(x, \xi^{\prime}\right)\right| \leq C_{\alpha \beta} \Phi_{l}^{-|\beta|}\left(x, \xi^{\prime}\right) \varphi_{l}^{1-|\alpha|}\left(x, \xi^{\prime}\right) .
$$

One can deduce these from (2.16) by using the following lemma which is frequently used in the proof of Lemma 2.3 .

Lemma 2.2. Let $U \subset \boldsymbol{R}^{n}$ and $V \subset \boldsymbol{R}^{m}$ be open sets, let $F \in C^{\infty}(U)$, and let $f: V \rightarrow U$ be $C^{\infty}$-mapping with $f=\left(f_{1}, \cdots, f_{n}\right)$. Suppose that there exist positive functions $Z(y), N_{j}(y)(j=1, \cdots, n)$ on $U$, and $M_{\alpha}(x)\left(\alpha \in Z_{+}^{m}\right)$ on $V$ with $M_{\alpha+\beta}$ $=M_{\alpha} M_{\beta}$ satisfying

$$
\begin{aligned}
& |F|_{\alpha}:=\sup _{y \in U}\left|\partial^{\alpha} F(y)\right| Z(y)^{-1} N(y)^{\alpha}<+\infty, \\
& \left|f_{j}\right|_{\alpha}:=\sup _{x \in V}\left|\partial^{\alpha} f_{j}(x)\right| M_{\alpha}(x)^{-1} N_{j}(f(x))^{-1}<+\infty .
\end{aligned}
$$

Set

$$
|F|_{L}=\max _{|\alpha| \leq L}|F|_{\alpha}, \quad|f|_{\alpha}=\max _{i=1, \cdots, n} \max _{\beta \leq \alpha}\left|f_{i}\right|_{\beta}
$$

Then

$\left|\partial^{\alpha}(F \circ f)(x)\right| \leq|F|_{|\alpha|}\left(|f|_{\alpha}+1\right)^{|\alpha|} n^{|\alpha|} 2^{(|\alpha|(|\alpha|-1)) / 2} Z(f(x)) M_{\alpha}(x)$ for any $\alpha$.

Proof of this lemma is straightforward.

Now we define a symbol class for a pair of positive $C^{\infty}$ functions $\Phi$ and $\varphi$ on $\boldsymbol{R}^{n} \times \boldsymbol{R}^{n-1}$ satisfying that

(i) there exist $C>0$ and $c>0$ such that

$$
\begin{aligned}
& c\left(1+\left|\xi^{\prime}\right|\right)^{1 / 2} \leq \Phi\left(x, \xi^{\prime}\right) \leq C\left(1+\left|\xi^{\prime}\right|\right), \\
& c\left(1+\left|\xi^{\prime}\right|\right)^{-1 / 2} \leq \varphi\left(x, \xi^{\prime}\right) \leq C ;
\end{aligned}
$$

(ii) for any $\alpha \in \mathbb{Z}_{+}^{n}$ and $\beta \in Z_{+}^{n-1}$ there exists $C_{\alpha \beta}>0$ such that

$$
\begin{aligned}
& \left|\partial_{x}^{\alpha} \partial_{\xi^{\prime}}^{\beta} \Phi\left(x, \xi^{\prime}\right)\right| \leq C_{\alpha \beta} \Phi\left(x, \xi^{\prime}\right)^{1-|\beta|} \varphi\left(x, \xi^{\prime}\right)^{-|\alpha|}, \\
& \left|\partial_{x}^{\alpha} \partial_{\xi^{\prime}}^{\beta} \varphi\left(x, \xi^{\prime}\right)\right| \leq C_{\alpha \beta} \Phi\left(x, \xi^{\prime}\right)^{-|\beta|} \varphi\left(x, \xi^{\prime}\right)^{1-|\alpha|} ;
\end{aligned}
$$

(iii) there exists $C^{\prime}>0$ such that

$$
C^{\prime-1}\left(1+\left|\xi^{\prime}\right|\right) \leq \frac{\Phi\left(x, \xi^{\prime}\right)}{\varphi\left(x, \xi^{\prime}\right)} \leq C^{\prime}\left(1+\left|\xi^{\prime}\right|\right) .
$$

For $M, m \in \boldsymbol{R}$ we say that a function $a \in C^{\infty}\left(\boldsymbol{R}^{n} \times \boldsymbol{R}^{n-1}\right)$ belongs to the set $S_{\phi, \varphi}^{M, m}$ if $a$ satisfies the estimates that for any $\alpha, \beta$ there exists $C_{\alpha \beta}>0$ such that

$$
\left|\partial_{x}^{\alpha} \partial_{\xi^{\prime}}^{\beta} a\left(x, \xi^{\prime}\right)\right| \leq C_{\alpha \beta} \Phi^{M-|\beta|}\left(x, \xi^{\prime}\right) \varphi^{m-|\alpha|}\left(x, \xi^{\prime}\right) .
$$

$\Phi_{l}$ and $\varphi_{l}$ in Lemma 2.1 satisfy $(2.31) \sim(2.33)$ for each $l$. Now we shall prove 
the main lemma in this section. This gives a local factorization of $P$ in Proposition 1.1 into first order factors for which Carleman estimates are deduced in Proposition 3.2.

Lemma 2.3. One can find two families of a finite number of $C^{\infty}$ functions on $\mathbb{R}^{n} \times \mathbb{R}^{n-1},\left\{\psi_{j k}\right\}_{k \in I}(j=0,1)$ with $\psi_{j k} \neq 0$ for any $j, k$ having the following properties.

(1) $\left\{\psi_{1 k}\right\}_{k \in I}$ is a finite partitions of unity of $\mathbb{R}^{n} \times \mathbb{R}^{n-1}$.

(2) $\psi_{0 k}=1$ on a neighbourhood of supp $\psi_{1 k}$.

(3) For each $k \in \mathbb{I}$ there exists $l \in\{1,2\}$ such that $\psi_{j k} \in S_{\Phi_{l}, \varphi_{l}}^{0,0}$ and one of the following (I), (II), (III) holds.

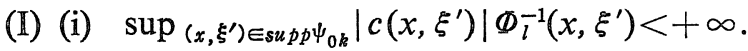

$p_{l}(x, \xi)=\mathbb{I}_{j=1}^{3}\left(\xi_{1}-\lambda_{l}\left(x, \xi^{\prime}\right)-\Lambda_{j}\left(x, \xi^{\prime}\right)\right)$ on a neighbourhood of supp $\psi_{0 k}$ as polynomials in $\xi_{1}$ where $\Lambda_{j} \in S_{\mathscr{l}_{l}, \varphi_{l}}^{1,0}$ depending on $k$ with

(ii) $\inf _{\left|\xi^{\prime}\right| \geq R}\left|\operatorname{Im}\left(\lambda_{l}\left(x, \xi^{\prime}\right)+\Lambda_{j}\left(x, \xi^{\prime}\right)\right)\right|\left\langle\xi^{\prime}\right\rangle^{-1}>0$ for some $R>0$,

(iii) $\inf _{\left(x, \xi^{\prime}\right) \in \text { supp } \psi_{0 k}}\left|\Lambda_{j}\left(x, \xi^{\prime}\right)-\Lambda_{j^{\prime}}\left(x, \xi^{\prime}\right)\right| \Phi_{l}^{-1}\left(x, \xi^{\prime}\right)>0$ if $j \neq j^{\prime}$.

(II) $p_{l}(x, \xi)=\left(\xi_{1}-\lambda_{l}\left(x, \xi^{\prime}\right)-c_{l}\left(x, \xi^{\prime}\right)-\Lambda_{1}\left(x, \xi^{\prime}\right)\right) \mathbb{I}_{j=2}^{3}\left(\xi_{1}-\lambda_{l}\left(x, \xi^{\prime}\right)-\Lambda_{j}\left(x, \xi^{\prime}\right)\right)$ on a neighbourhood of supp $\psi_{0 k}$ as polynomials in $\xi_{1}$ where $\mathbb{A}_{j} \in S_{\phi_{l}^{\prime}, \varphi_{l}}^{1,0}$ depending on $k$ with

(i) $\inf _{\left|\xi^{\prime}\right| \geq R}\left|\operatorname{Im}\left(\lambda_{l}\left(x, \xi^{\prime}\right)+\Lambda_{j}\left(x, \xi^{\prime}\right)\right)\right|\left\langle\xi^{\prime}\right\rangle^{-1}>0$ for some $R>0$ if $j=2,3$,

(ii) $\left.\inf _{\left|\xi^{\prime}\right| \geq R}\left|\operatorname{Im}\left(\lambda_{l}\left(x, \xi^{\prime}\right)+c_{l}\left(x, \xi^{\prime}\right)+\Lambda_{1}\left(x, \xi^{\prime}\right)\right)\right|\left\langle\xi^{\prime}\right\rangle^{-1}\right\rangle 0$ for some $R>0$,

(iii) $\inf _{\left(x, \xi^{\prime}\right) \in \text { supp } \psi_{0 k}}\left|c\left(x, \xi^{\prime}\right)\right| \Phi_{l}^{-1}\left(x, \xi^{\prime}\right)>0$,

(iv) $\inf _{\left(x, \xi^{\prime}\right) \in s u p p \psi_{0 k}}\left|c_{l}\left(x, \xi^{\prime}\right)+\Lambda_{1}\left(x, \xi^{\prime}\right)-\Lambda_{j}\left(x, \xi^{\prime}\right)\right|\left|c\left(x, \xi^{\prime}\right)\right|^{-1}>0$ if $j=2,3$,

(v) $\Lambda_{2}\left(x, \xi^{\prime}\right) \neq \Lambda_{3}\left(x, \xi^{\prime}\right)$ for $\left(x, \xi^{\prime}\right) \in \operatorname{supp} \psi_{0 k}$,

there exists an open set $U$ containing supp $\psi_{0 k}$ such that

(vi) $\sup _{\left(x, \xi^{\prime}\right) \in U}\left|\partial_{x}^{\alpha} \partial_{\xi^{\prime}}^{\beta} \Lambda_{j}\left(x, \xi^{\prime}\right)\right|\left|\Lambda_{2}\left(x, \xi^{\prime}\right)-\Lambda_{3}\left(x, \xi^{\prime}\right)\right|^{-1} \Phi_{l}^{-|\beta|}\left(x, \xi^{\prime}\right) \varphi_{l}^{-|\alpha|}\left(x, \xi^{\prime}\right)$ $<+\infty$ for $j=2,3$ and $\alpha \in \mathbb{Z}_{+}^{n-1}, \beta \in \mathbb{Z}_{+}^{n-1}$.

(III) $\Phi_{l}\left(x, \xi^{\prime}\right) \leq C\left\langle\xi^{\prime}\right\rangle^{1 / 2}$ on supp $\psi_{0 k}$ for some $C>0$.

Proof. Step 1. In this step we shall deduce the algebraic equations with parameters to decompose $p_{l}$. Set

$$
\begin{aligned}
& D_{l}=\left\{\left(x, \xi^{\prime}\right) \in \mathbb{R}^{n} \times \mathbb{R}^{n-1} ;\left\langle g_{l^{\prime}}\left(x, \xi^{\prime}\right)\right\rangle>\frac{1}{100}\left\langle g_{l}\left(x, \xi^{\prime}\right)\right\rangle\right\} \quad\left(l \neq l^{\prime}\right), \\
& \left.\mathscr{D}_{l 2}(\varepsilon)=\left\{(x, \xi) \in \mathbb{R}^{n} \times \mathbb{R}^{n-1} ; \varepsilon\left\langle c\left(x, \xi^{\prime}\right)\right\rangle^{3}\right\rangle\left\langle g_{l}\left(x, \xi^{\prime}\right)\right\rangle\right\}, \\
& \mathscr{D}_{l 2}(\varepsilon)=\left\{\left(x, \xi^{\prime}\right) \in \mathbb{R}^{n} \times \mathbb{R}^{n-1} ;\left\langle g_{l}\left(x, \xi^{\prime}\right)\right\rangle>\frac{\varepsilon}{10}\left\langle c\left(x, \xi^{\prime}\right)\right\rangle\right\} \\
& \Gamma_{l}(N)=\left\{\left(x, \xi^{\prime}\right) \in \mathbb{R}^{n} \times \mathbb{R}^{n-1} ;\left\langle g_{l}\left(x, \xi^{\prime}\right)\right\rangle>N\left\langle\xi^{\prime}\right\rangle^{3 / 2}\right\} \\
& D_{l j}(\varepsilon, N)=\Gamma_{l}(N) \cap \mathscr{D}_{l j}(\varepsilon) \cap D_{l},
\end{aligned}
$$




$$
\Gamma_{0 l}(N)=\left\{\left(x, \xi^{\prime}\right) \in \boldsymbol{R}^{n} \times \boldsymbol{R}^{n-1} ;\left\langle g_{l}\left(x, \xi^{\prime}\right)\right\rangle\left\langle 2 N\left\langle\xi^{\prime}\right\rangle^{3 / 2}\right\},\right.
$$

where $0<\varepsilon<1, N>1$ which are determined in Step 3.

We assume $N \geq 500$. Then

$$
\begin{gathered}
\left|c\left(x, \xi^{\prime}\right)\right|>1 \text { on } D_{l 1}(\varepsilon, N), \\
\min \left\{\left|g_{j}\left(x, \xi^{\prime}\right)\right| ; j=1,2\right\}>1 \text { on } \Gamma_{l}(N) \cap D_{l} .
\end{gathered}
$$

We have

$$
\begin{aligned}
& p_{l}(x, \xi)=c_{l}\left(x, \xi^{\prime}\right)^{3} f_{1}\left(Z_{l}(x, \xi), w_{l}\left(x, \xi^{\prime}\right)\right) \quad \text { for } \quad\left(x, \xi^{\prime}\right) \in D_{l 1}(\varepsilon, N), \\
& p_{l}(x, \xi)=\left|g_{l}\left(x, \xi^{\prime}\right)\right| f_{2}\left(\tilde{Z}_{l}(x, \xi), \tilde{w}_{l}\left(x, \xi^{\prime}\right)\right) \text { for } \quad\left(x, \xi^{\prime}\right) \in D_{l 2}(\varepsilon, N),
\end{aligned}
$$

where

$$
\begin{aligned}
& f_{1}(z, w)=z^{3}(z-1)+w_{2} w_{1}^{3}, \\
& f_{2}(z, w)=z^{2}\left(z-w_{2}\right)+w_{1},
\end{aligned}
$$

for $z \in \boldsymbol{C}, w=\left(w_{1}, w_{2}\right) \in \boldsymbol{C}^{2}$, and by definition,

$$
\begin{aligned}
& Z_{l}(x, \xi)=c_{l}\left(x, \xi^{\prime}\right)^{-1}\left(\xi_{1}-\lambda_{l}\left(x, \xi^{\prime}\right)\right), \\
& w_{l}\left(x, \xi^{\prime}\right) \equiv\left(w_{l 1}\left(x, \xi^{\prime}\right), w_{l 2}\left(x, \xi^{\prime}\right)\right)=\left(\frac{\left|g_{l}\left(x, \xi^{\prime}\right)\right|^{1 / 3}}{c_{l}\left(x, \xi^{\prime}\right)}, \frac{g_{l}\left(x, \xi^{\prime}\right)}{\left|g_{l}\left(x, \xi^{\prime}\right)\right|}\right)
\end{aligned}
$$

for $\left(x, \xi^{\prime}\right) \in D_{l 1}(\xi, N)$,

$$
\begin{aligned}
& \tilde{Z}_{l}(x, \xi)=\left|g_{l}\left(x, \xi^{\prime}\right)\right|^{-1 / 3}\left(\xi_{1}-\lambda_{l}\left(x, \xi^{\prime}\right)\right) \\
& \tilde{w}_{l}\left(x, \xi^{\prime}\right) \equiv\left(\tilde{w}_{l 1}\left(x, \xi^{\prime}\right), \tilde{w}_{l 2}\left(x, \xi^{\prime}\right)\right)=\left(\frac{g_{l}\left(x, \xi^{\prime}\right)}{\left|g_{l}\left(x, \xi^{\prime}\right)\right|}, \frac{c_{l}\left(x, \xi^{\prime}\right)}{\left|g_{l}\left(x, \xi^{\prime}\right)\right|^{1 / 3}}\right)
\end{aligned}
$$

for $\left(x, \xi^{\prime}\right) \in D_{l 2}(\varepsilon, N)$. We have

$$
\begin{array}{ll}
\left|w_{l 2}\left(x, \xi^{\prime}\right)\right|=1, & \left|w_{l 1}\left(x, \xi^{\prime}\right)\right| \leq 2 \varepsilon^{1 / 3}, \\
\left|\tilde{w}_{l 1}\left(x, \xi^{\prime}\right)\right|=1, & \left|\tilde{w}_{l 2}\left(x, \xi^{\prime}\right)\right| \leq 20 \varepsilon^{-1 / 3} .
\end{array}
$$

If we denote by $D(w)$ the descriminant of polynomial $f_{2}(z, w)$ of $Z$, we have

$$
D(w)=\left(27 w_{1}-4 w_{2}^{3}\right) w_{1}
$$

and

$$
D\left(\tilde{w}_{l}\left(x, \xi^{\prime}\right)\right)=27\left|g_{l}\left(x, \xi^{\prime}\right)\right|^{-2}\left(g_{l}\left(x, \xi^{\prime}\right)+(-1)^{l+1} \frac{4}{27} c\left(x, \xi^{\prime}\right)^{3}\right) g_{l}\left(x, \xi^{\prime}\right) .
$$

Using the equality

$$
g_{l}+(-1)^{l+1} \frac{4}{27} c^{3}=g_{l^{\prime}}+(-1)^{l} c\left(-\frac{2}{3} a_{1}+\frac{4}{9} c a_{2}\right) \text { if } l \neq l^{\prime}
$$


we see that there exists a constant $C_{0}$ such that

$$
\left|D\left(\tilde{w}_{l}\left(x, \xi^{\prime}\right)\right)\right| \geq \frac{1}{20} \quad \text { if } \quad N^{2} \varepsilon \geq C_{0} \text { and } N \geq 500 .
$$

Step 2. In this step we factorize $f_{i}(z, w)$ as a polynomial in $z$ locally. We first consider $f_{1}(z, w)$. From the implicit function theorem there exists $\delta_{0}>0$ such that for any pair of positive numbers $\delta, R$ with $\delta R^{3}<\delta_{0}$ there exist holomorphic functions $\mu_{1}, A, B$ in $w \in B_{\delta}^{1}(0) \times B_{R}^{1}(0)$ satisfying

$$
f_{1}(z, w)=\left(z-\mu_{1}(w)\right)\left(z^{2}+2 A(w) z+B(w)\right) \text { for } \quad w \in B_{\delta}^{1}(0) \times B_{R}^{1}(0)
$$

with $\mu_{1}\left(0, w_{2}\right)=1, \mu_{1}(w) \neq 0$ everywhere, $A\left(0, w_{2}\right)=B\left(0, w_{2}\right)=0$. Here, $B_{r}^{m}(0)$ denotes the open ball with the center at the origin in $\mathbb{C}^{m}$ and the radius $r$. A simple calculation shows that there exists a holomorphic function $D(w)$ on $B_{\delta}^{1}(0) \times B_{R}^{1}(0)$ satisfying

$$
A(w)^{2}-B(w)=w_{1}^{3} D(w), D\left(0, w_{2}\right)=-w_{2} .
$$

We take $\delta, R$ as $\delta=\left(\delta_{0} / 4\right)^{1 / 3}, R=3$.

Then one can choose a positive number $\delta_{1}$ with $\delta_{1}<\left(\delta_{0} / 4\right)^{1 / 3}$ and an open covering $\left\{U_{1 j}\right\}_{j=1}^{k_{1}}$ of $\left.\overline{\left(B_{\delta_{1} / 2}^{1}(0)\right.} \backslash\{0\}\right) \times \overline{B_{1 / 2}^{1}(0)^{c} \cap B_{2}^{1}(0)}$ in $C \backslash\{0\} \times \mathbb{C}$ such that

$$
\begin{aligned}
& U_{1 j}=\left(B_{\delta_{1}}^{1}(0) \cap U_{1}\right) \times B_{1 j}, \quad j=1, \cdots, k_{1} / 2 \quad\left(k_{1} \text { is even }\right) \\
& U_{1 j}=\left(B_{\delta_{1}}^{1}(0) \cap U_{2}\right) \times B_{1 j}, \quad j=k_{1} / 2+1, \cdots, k_{1}
\end{aligned}
$$

where $U_{1}, U_{2}$ are two connected open sets in $C$ with angles $<2 \pi$ such that $U_{1} U$ $U_{2}=C \backslash\{0\}$ and $B_{1 j}$ are open sets in $B_{3}^{1}(0) \backslash\{0\}$ with $\bigcup_{j=1}^{k_{1}} B_{1 j} \supseteq \overline{B_{1 / 2}^{1}(0)^{c} \cap B_{2}^{1}(0)}$, and such that there exist holomorphic functions $\mu_{1 j k}(w)$ in $U_{1 j}, k=1,2$ satisfying

$$
\begin{gathered}
f_{1}(z, w)=\left(z-\mu_{1}(w)\right) \prod_{k=1}^{2}\left(z-\mu_{1 j k}(w)\right), w \in U_{1 j}, \\
C_{1}\left|\mu_{1 j 1}(w)-\mu_{1 j 2}(w)\right| \geq|w|^{2 / 3}, \\
C_{1}\left|\mu_{1}(w)-\mu_{1 j k}(w)\right| \geq 1, \\
\left|\partial_{w}^{\alpha} \mu_{1 j k}(w)\right| \leq\left|w_{1}\right|^{(3 / 2)-\alpha_{1}} .
\end{gathered}
$$

We also note that

$$
\left|\partial_{w}^{\alpha}\left(1-\mu_{1}(w)\right)\right|\left|w_{1}\right|^{\alpha_{1}} \leq C_{a}^{\prime}\left|w_{1}\right|^{3} \text { for } w \in B_{\delta_{1}}^{1}(0) \times B_{3 / 2}^{1}(0) .
$$

Next we take up $f_{2}(z, w)$. We set for $R^{\prime}>0, \delta^{\prime}>0$ which are to be determined in the next step

$$
K\left(R^{\prime}, \delta^{\prime}\right)=\left\{w \in C^{2} ;|D(w)| \geq \delta^{\prime},|w| \leq R^{\prime}\right\} .
$$


One can find open balls $U_{2 j}, j=1, \cdots, k_{2}$ in $\boldsymbol{C}^{2}$ and holomorphic functions $\mu_{2 j k}$ on a neighbourhood of $U_{2 j}, k=1,2,3, j=1, \cdots, k_{2}$ such that

$$
\begin{gathered}
|D(w)| \geq \frac{\delta^{\prime}}{2} \quad \text { on } \quad U_{2 j} \text { and } K\left(R^{\prime}, \delta^{\prime}\right) \subseteq \bigcup_{j=1}^{k_{2}} U_{2 j}, \\
f_{2}(z, w)=\prod_{k=1}^{3}\left(z-\mu_{2 j k}(w)\right) \text { for } w \in U_{2 j} .
\end{gathered}
$$

Step 3. In this step we shall define a family of non-negative functions in $\bigcup_{l=1}^{2} S_{\Phi_{l}, \varphi_{l}}^{0,0}$ where sum is greater than or equal to 1 such that on the support of each one, one of (I), (II), (III), in Lemma 2.3 will holds. We take $\varepsilon, N$ as $\varepsilon=$ $\min \left\{\frac{1}{2},\left(\frac{\delta_{1}}{8}\right)^{3}\right\}, N=\max \left\{500,\left(C_{0} \varepsilon^{-1}\right)^{1 / 2}\right\}$, and we take $R^{\prime}, \delta^{\prime}$ in the Step 2 as $R^{\prime}=\left(1+\left(\frac{20}{\varepsilon}\right)^{2 / 3}\right)^{1 / 2}, \delta^{\prime}=\frac{1}{20}$. We denote $\mathscr{D}_{l j}, \Gamma_{l}(N), D_{l j}(\varepsilon, N)$ by $\mathscr{D}_{l j}, \Gamma_{l}, D_{l j}$. Choose $\chi_{i} \in C^{\infty}(\boldsymbol{R}), i=0,1,2$ so that $0 \leq \chi \leq 1$, supp $\chi_{i} \subseteq(1, \infty), \chi_{i}=1$ on $[2, \infty)$, $\chi_{i}=1$ on a neighbourhood of $\operatorname{supp} \chi_{i+1}$ for $i=0,1$. Define $C^{\infty}$ functions $\Psi_{i}^{(l, s)}\left(x, \xi^{\prime}\right)$ on $\boldsymbol{R}^{n} \times \boldsymbol{R}^{n-1}$ for $i=0,1,2$ and $l=1,2$ by

$$
\begin{aligned}
& \Psi_{i}^{(l, 1)}=\chi_{i}\left(\varepsilon\langle c\rangle^{3} /\left\langle g_{l}\right\rangle\right) \chi_{i}\left(100\left\langle g_{l^{\prime}}\right\rangle\left\langle\left\langle g_{l}\right\rangle\right) \chi_{i}\left(N^{-1}\left\langle g_{l}\right\rangle\left\langle\xi^{\prime}\right\rangle^{-3 / 2}\right),\right. \\
& \Psi_{i}^{(l, 2)}=\chi_{i}\left(\frac{10}{\varepsilon}\left\langle g_{l}\right\rangle \mid\langle c\rangle^{3}\right) \chi_{i}\left(100\left\langle g_{l^{\prime}}\right\rangle\left\langle\left\langle g_{l}\right\rangle\right) \chi_{i}\left(N^{-1}\left\langle g_{l}\right\rangle\left\langle\xi^{\prime}\right\rangle^{-3 / 2}\right),\right.
\end{aligned}
$$

where $l \neq l^{\prime}$.

Then we have that supp $\Psi_{i}^{(l, s)} \subseteq D_{l s}$ and that

$$
\sum_{s=1}^{2} \Psi^{(l, s)} \geq 1 \quad \text { on } \quad \Gamma_{l}(2 N) \cap \tilde{D}_{l}
$$

where $\widetilde{D}_{l}=\left\{\left(x, \xi^{\prime}\right) \in \boldsymbol{R}^{n} \times \boldsymbol{R}^{n-1} ;\left\langle g_{l^{\prime}}\left(x, \xi^{\prime}\right)\right\rangle>\frac{1}{50}\left\langle g_{l}\left(x, \xi^{\prime}\right)\right\rangle\right\}$. Now we define $\tilde{\Psi}_{i}^{(l, s, j)}, \Psi_{i}^{(l, 0)}, \Lambda_{k}^{(l, s, j)} \in C^{\infty}\left(\boldsymbol{R}^{n} \times \boldsymbol{R}^{n-1}\right)(i=0,1,2 ; l, s=1,2 ; k=1,2,3 ; j=1, \cdots$, $k_{s}$ ) with notations $k_{1}, k_{2}$ in Step 2 where $\tilde{\Psi}_{i}^{(l, s, j)}, \Psi_{i}^{(l, 0)}$ are functions stated in the beggining of this step such that (II) (resp. (I)) in Lemma 2.3 holds on supp $\tilde{\Psi}_{i}^{(l, 1, j)}$ (resp. supp $\Psi_{i}^{(l, 2, j)}$ ) and (III) holds on supp $\Psi_{i}^{(l, 0)}$, and where $\Lambda_{k}^{(l, 1, j)}$ (resp. $\Lambda_{k}^{(l, 2, j)}$ ) corresponds to $\Lambda_{k}$ in the case (II) (resp. (I)).

To do so we choose $\varphi_{i 1 j} \in C^{\infty}(\boldsymbol{C} \backslash\{0\} \times \boldsymbol{C}), i=0,1,2, j=1, \cdots, k_{1}$ and $\varphi_{i 2 j} \in$ $C_{0}^{\infty}\left(U_{2 j}\right), i=0,1,2, j=1, \cdots, k_{2}$ so that

$\operatorname{supp} \varphi_{i s j} \subseteq U_{s j} ; \varphi_{i s j}=1$ on a neighbourhood of $\operatorname{supp} \varphi_{i+1 s j}$ for $i=0,1$;

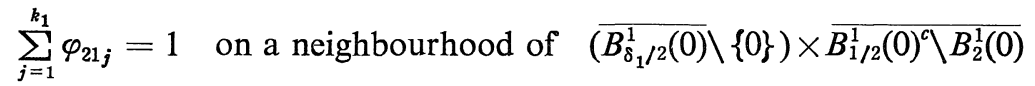




$$
\begin{gathered}
\text { in } C \backslash\{0\} \times C ; \\
\sum_{j=1}^{k_{2}} \varphi_{22 j}=1 \quad \text { on a neighbourhood of } K\left(R^{\prime}, \delta^{\prime}\right) ; \\
\left|\partial_{w}^{\alpha} \varphi_{i 1 j}(w)\right| \leq C_{a}^{\prime}\left|w_{1}\right|^{-\alpha_{1}} ; 0 \leq \varphi_{i s j} \leq 1
\end{gathered}
$$

$\tilde{\Psi}_{i}^{(l, s, j)}, \Lambda_{k}^{(l, s, j)}$ are defined as follows.

$$
\tilde{\Psi}_{i}^{(l, s, j)} \equiv 0, \quad \Lambda_{k}^{(l, s, j)} \equiv 0 \quad \text { when } \quad D_{l s}=\emptyset .
$$

When $D_{l 1} \neq \emptyset$,

$$
\begin{aligned}
& \tilde{\Psi}_{i}^{(l, s, j)}\left(x, \xi^{\prime}\right)= \begin{cases}\varphi_{i 1 j}\left(w_{l}\left(x, \xi^{\prime}\right)\right) \Psi_{i}^{(l, 1)}\left(x, \xi^{\prime}\right) & \left(x, \xi^{\prime}\right) \in D_{l 1}, \\
0 & \text { otherwise }\end{cases} \\
& \Lambda_{1}^{(l, 1, j)}\left(x, \xi^{\prime}\right)= \begin{cases}c_{l}\left(x, \xi^{\prime}\right)\left(\mu_{1}\left(w_{l}\left(x, \xi^{\prime}\right)\right)-1\right) \tilde{\Psi}_{0}^{(l, 1, j)}\left(x, \xi^{\prime}\right) & \left(x, \xi^{\prime}\right) \in D_{l 1}, \\
0 & \text { otherwise }\end{cases} \\
& \Lambda_{k}^{(l, 1, j)}\left(x, \xi^{\prime}\right)= \begin{cases}c_{l}\left(x, \xi^{\prime}\right) \mu_{1 j k}\left(w_{l}\left(x, \xi^{\prime}\right)\right) \tilde{\Psi}_{0}^{(l, 1, j)}\left(x, \xi^{\prime}\right) & \left(x, \xi^{\prime}\right) \in w_{l}^{-1}\left(U_{1 j}\right) \\
0 & \text { otherwise }\end{cases}
\end{aligned}
$$

for $k=2,3$.

When $D_{l 2} \neq \emptyset$,

$$
\begin{gathered}
\tilde{\Psi}_{i}^{(l, 2, j)}\left(x, \xi^{\prime}\right)= \begin{cases}\varphi_{i 2 j}\left(\tilde{w}_{l}\left(x, \xi^{\prime}\right)\right) \Psi_{i}^{(l, 2)}\left(x, \xi^{\prime}\right) & \left(x, \xi^{\prime}\right) \in D_{l 2}, \\
0 & \text { otherwise }\end{cases} \\
\Lambda_{k}^{(l, 2, j)}\left(x, \xi^{\prime}\right)= \begin{cases}\left|g_{l}\left(x, \xi^{\prime}\right)\right|^{1 / 3} \mu_{2 j k}\left(\tilde{w}_{l}\left(x, \xi^{\prime}\right)\right) \tilde{\Psi}_{0}^{(l, 2, j)}\left(x, \xi^{\prime}\right) & \left(x, \xi^{\prime}\right) \in \tilde{w}_{l}^{-1}\left(U_{2 j}\right) 。 \\
0 & \text { otherwise }\end{cases}
\end{gathered}
$$

$\Psi_{i}^{(l, 0)}$ is defined by

$$
\Psi_{i}^{(l, 0)}=\left(1-\chi_{2-i}\right)\left(5^{-1}\left\langle g_{l}\right\rangle\left\langle\xi^{\prime}\right\rangle^{-3 / 2} N^{-1}\right) .
$$

Since $\sum_{j=1}^{k_{s}} \tilde{\Psi}_{i}^{(l, s, j)} \geq \Psi_{i}^{(l, s)}$ from the definition of $\varphi_{i s j}$, and since $\bigcap_{l=1}^{2} \Gamma_{l}(2 N) \subseteq$ $\bigcup_{l=1}^{2}\left(\Gamma_{l}(2 N) \cap \widetilde{D}_{l}\right)$, we have that

$$
\sum_{l=1}^{2} \sum_{s=1}^{2} \sum_{j=1}^{k_{s}} \tilde{\Psi}_{i}^{(l, s, j)} \geq 1 \quad \text { on } \bigcap_{l=1}^{2} \Gamma_{l}(2 N)
$$

in view of (2.48). Thus, since $\cup_{l=1}^{2} \Gamma_{0 l}\left(\frac{5}{2} N\right) \cup \cap_{l=1}^{2} \Gamma_{l}(2 N)=\mathbb{R}^{n} \times \mathbb{R}^{n-1}$, we have that

$$
\sum_{l=1}^{2}\left(\sum_{s=1}^{2} \sum_{j=1}^{k_{s}} \tilde{\Psi}_{i}^{(l, s, j)}+\Psi_{i}^{(l, 0)}\right) \geq 1
$$

Since $\tilde{\Psi}_{i=1}^{(l, s, j)}=1$ on a neighbourhood of supp $\tilde{\Psi}_{i}^{(l, s, j)}$ for $i=1,2$, from (2.35) and (2.36) we have that the factorization in (i) (resp. (ii)) in Lemma 2.3 holds for $\left(x, \xi^{\prime}\right) \in \operatorname{supp} \tilde{\Psi}_{1}^{(1,2, j)}$ (resp. supp $\tilde{\Psi}_{1}^{(l, 1, j)}$ ) with $\Lambda_{k}$ replaced by $\Lambda_{k}^{(l, 2, j)}$ (resp. $\Lambda_{k}^{(l, 1, j)}$ )。 
Step 4. In this step we deduce the estimates of derivatives of functions defined in Step 3. To do so, we have to deduce the estimates of derivatives of functions $\left.\left\langle g_{l}\right\rangle\left\langle\xi^{\prime}\right\rangle^{-3 / 2}\right|_{\Gamma_{l}},\langle c\rangle^{3}\left|\left\langle g_{l}\right\rangle\right|_{D_{l 1}},\left\langle g_{l^{\prime}}\right\rangle\left|\left\langle g_{l}\right\rangle\right|_{\Gamma_{l} \cap D_{l}},\left\langle g_{l}\right\rangle\left|\langle c\rangle^{3}\right| D_{l 2} \cap \mathscr{D}_{l 1}, w_{l}, \tilde{w}_{l}$.

Definition 2.1. For an open set $U$ in $\boldsymbol{R}^{n} \times \boldsymbol{R}^{n-1}$ and a positive function $Z\left(x, \xi^{\prime}\right)$ on $U$ we set

$S_{l}(U, Z)=\left\{a \in C^{\infty}(U) ;\left|a_{(\alpha)}^{(\beta)}\left(x, \xi^{\prime}\right)\right| \leq C_{\alpha \beta} M_{\alpha, \beta}^{(l)}\left(x, \xi^{\prime}\right) Z(x, \xi)\right.$ for any $\left.\alpha, \beta\right\}$ where $a_{(\alpha)}^{(\beta)}=\partial_{x}^{\alpha} \partial_{\xi^{\prime}}^{\beta}$ a and $M_{\alpha, \beta}^{(l)}=\varphi_{l}^{-|\alpha|} \Phi_{l}^{-|\beta|}$.

Let us consider $g_{l}$ on $\Gamma_{l}$. Since $\Phi_{l}^{-1} \geq\left\langle g_{l}\right\rangle^{-1 / 3} \geq \sqrt{2}^{-1 / 3}\left|g_{l}\right|^{-1 / 3}$ on $\Gamma_{l}$, Lemma 2.1 implies that

$$
\left.g_{l}\right|_{\Gamma_{l}} \in S_{l}\left(\Gamma_{l},\left.\left|g_{l}\right|\right|_{\Gamma_{l}}\right) \text { when } \Gamma_{l} \neq \emptyset .
$$

When $\Gamma_{l} \neq \emptyset$, taking in Lemma $2.2 U=\Gamma_{l}, V=\boldsymbol{R}^{2} \backslash\{0\}, f=\left(\operatorname{Re}\left[\left.g_{l}\right|_{\Gamma_{l}}\right], \operatorname{Im}\left[\left.g_{l}\right|_{\Gamma_{l}}\right]\right)$, $F(y)=Z(y)=|y|^{s}(s \in \boldsymbol{R}), N_{i}(y)=|y|, M_{(\alpha, \beta)}=\left.M_{\alpha, \beta}^{(l)}\right|_{\Gamma_{l}}$ one obtain that

$$
\left.\left|g_{l}\right|^{s}\right|_{\Gamma_{l}} \in S_{l}\left(\Gamma_{l},\left.\left|g_{l}\right|^{s}\right|_{\Gamma_{l}}\right) \text {. }
$$

When $\Gamma_{l} \neq \emptyset$, one also obtain taking in Lemma $2.2 U=\Gamma_{l}, V=(0,+\infty), f=$ $\left.\left|g_{l}\right|\right|_{\Gamma_{l}}, F(y)=\langle y\rangle^{s / 2}, N_{1}(y)=\langle y\rangle, M_{(\alpha, \beta)}=\left.M_{\alpha, \beta}^{(l)}\right|_{\Gamma_{l}}$ that

$$
\left.\left\langle g_{l}\right\rangle^{s}\right|_{\Gamma_{l}} \in S_{l}\left(\Gamma_{l},\left.\left\langle g_{l}\right\rangle^{s}\right|_{\Gamma_{l}}\right) \text {. }
$$

Next we consider $c$ on $D_{l 1}$. Noting the inequalities

$$
\begin{array}{rll}
\left\langle\xi^{\prime}\right\rangle \leq C|c| \varphi_{l}^{-1} & \text { on } & D_{l 1}, \\
1 \leq C|c| \Phi_{l}^{-1} & \text { on } & D_{l 1},
\end{array}
$$

we see that $\left\langle\xi^{\prime}\right\rangle^{1-|\beta|} \leq C_{\alpha \beta}|c| M_{\alpha, \beta}^{(l)}$ on $D_{l 1}$ when $|\alpha|+|\beta| \neq 0$. Thus we obtain

$$
\left.c\right|_{D_{l 1}} \in S_{l}\left(D_{l 1},\left.|c|\right|_{D_{l 1}}\right) \text { when } D_{l 1} \neq \emptyset \text {. }
$$

From this and Lemma 2.2, we see that

$$
\begin{gathered}
\left.\langle c\rangle^{s}\right|_{D_{l 1}} \in S_{l}\left(D_{l 1},\left.\langle c\rangle^{s}\right|_{D_{l 1}}\right) \text { when } D_{l 1} \neq \emptyset \\
\left(\left.c\right|_{D_{l 1}}\right)^{-k} \in S_{l}\left(D_{l 1},\left(\left.|c|\right|_{D_{l 1}}\right)^{-k}\right) \text { for } k \in Z \text { when } D_{l 1} \neq \emptyset .
\end{gathered}
$$

We need a lemma which follows from Leibniz rule.

Lemma 2.4. Let $Z_{i}\left(x, \xi^{\prime}\right)(i=1,2)$ be a positive function on an open set $U$ in $\boldsymbol{R}^{n} \times \boldsymbol{R}^{n-1}$, and let $a_{i} \in S_{l}\left(U, Z_{i}\right)(i=1,2)$. Then $a_{1} a_{2} \in S_{l}\left(U, Z_{1} Z_{2}\right)$.

From (2.51), (2.52), (2.57) the above lemma implies that when $D_{l 1} \neq \emptyset$, 


$$
w_{l 1} \in S_{l}\left(D_{l 1},\left|w_{l 1}\right|\right) \text { and } w_{l 2} \in S_{l}\left(D_{l 1}, 1\right) .
$$

Since on $\Gamma_{l}$ the estimates (2.54) with $|c|$ replaced by $\left|g_{l}\right|^{1 / 3}$ and $D_{l 1}$ by $\Gamma_{l}$ hold, we see that

$$
\left.c\right|_{D_{l 2}} \in S_{l}\left(D_{l 2},\left.\left|g_{l}\right|^{1 / 3}\right|_{D_{l 2}}\right) \text { when } D_{l 2} \neq \emptyset .
$$

From this, (2.51), and (2.52) Lemma 2.4 implies that

$$
\tilde{w}_{l j} \in S_{l}\left(D_{l 2}, 1\right) \text {. }
$$

Now from (2.53) and (2.56) Lemma 2.4 implies that

$$
\begin{gathered}
\left.\left\langle g_{l}\right\rangle\left\langle\xi^{\prime}\right\rangle^{-3 / 2}\right|_{\Gamma_{l}} \in S_{l}\left(\Gamma_{l},\left.\left\langle g_{l}\right\rangle\left\langle\xi^{\prime}\right\rangle^{-3 / 2}\right|_{\Gamma_{l}}\right) \text { when } \Gamma_{l} \neq \emptyset, \\
\langle c\rangle^{3}\left|\left\langle g_{l}\right\rangle\right|_{D_{l 2}} \in S_{l}\left(D_{l 2}, 1\right) \text { when } D_{l 2} \neq \emptyset .
\end{gathered}
$$

From (2.59), $c \mid D_{l 2} \cap D_{l 1} \in S_{l}\left(D_{l 2} \cap D_{l 1},\langle c\rangle \mid D_{l 2} \cap D_{l 1}\right)$ when $D_{l 2} \cap \mathscr{D}_{l 1} \neq \emptyset$ from which one obtain $\langle c\rangle^{s} \mid D_{l 2} \cap D_{l 1} \in S_{l}\left(D_{l 2} \cap D_{l 1},\langle c\rangle^{s} \mid D_{l 2} \cap D_{l 1}\right)$ by Lemma 2.2 when $D_{l 2} \cap D_{l 1} \neq \emptyset$. Thus from this and (2.53) Lemma 2.4 implies that

$$
\langle c\rangle^{3} /\left\langle g_{l}\right\rangle \mid D_{l 2} \cap D_{l 1} S_{l}\left(D_{l 2} \cap D_{l 1}, 1\right) \text {. }
$$

Since (2.53) with $\Gamma_{l}$ replaced by $\Gamma_{l}(N / 100)$ also holds because of the fact that $(N / 100)>1$, since $\Gamma_{l} \cap D_{l} \subseteq \Gamma_{l^{\prime}}(N / 100)$ if $l \neq l^{\prime}$, and since $M_{a, \beta}^{\left(l^{\prime}\right)} \leq 100^{(|\alpha|+|\beta|) / 3}$ $M_{a, \beta}^{(l)}$ on $\Gamma_{l} \cap D_{l}$, we see that $\left.\left\langle g_{l^{\prime}}\right\rangle\right|_{\Gamma_{l} \cap D_{l}} \in S_{l}\left(\Gamma_{l} \cap D_{l},\left.\left\langle g_{l^{\prime}}\right\rangle\right|_{\Gamma_{l} \cap D_{l}}\right)$ if $l \neq l^{\prime}$. From this and (2.53) Lemma 2.4 implies that

$$
\left\langle g_{l^{\prime}}\right\rangle\left|\left\langle g_{l}\right\rangle\right|_{\Gamma_{l} \cap D_{l}} \in S_{l}\left(\Gamma_{l} \cap D_{l},\left\langle g_{l^{\prime}}\right\rangle\left|\left\langle g_{l}\right\rangle\right|_{\Gamma_{l} \cap D_{l}}\right) \quad \text { when } \quad \Gamma_{l} \cap D_{l} \neq \emptyset \text {. }
$$

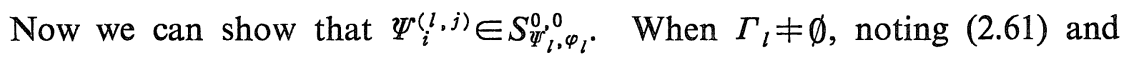
taking in Lemma $2.2 U=\Gamma_{l}, V=(0,+\infty), f=\left\langle g_{l}\right\rangle\left\langle\xi^{\prime}\right\rangle^{-3 / 2}, F(y)=\chi_{i}\left(N^{-1} y\right)$, $N_{1}(y)=y, Z(y)=1, M_{(\alpha, \beta)}=M_{\alpha, \beta}^{(l)}$ one obtain that

$$
\left.\chi_{i}\left(N^{-1}\left\langle g_{l}\right\rangle\left\langle\xi^{\prime}\right\rangle^{-3 / 2}\right)\right|_{\Gamma_{l}} \in S_{l}\left(\Gamma_{l}, 1\right) .
$$

Similar argument as above shows that

$$
\begin{gathered}
\left.\chi_{i}\left(\varepsilon\langle c\rangle^{3} /\left\langle g_{l}\right\rangle\right)\right|_{D_{l 1}} \in S_{l}\left(D_{l 1}, 1\right) \text { when } D_{l 1} \neq \emptyset, \\
\left.\chi_{i}\left(100\left\langle g_{l^{\prime}}\right\rangle \mid\left\langle g_{l}\right\rangle\right)\right|_{\Gamma_{l} \cap D_{l}} \in S_{l}\left(\Gamma_{l} \cap D_{l}, 1\right) \text { when } \Gamma_{l} \cap D_{l} \neq \emptyset, \\
\chi_{i}\left(\frac{10}{\varepsilon} \frac{\left\langle g_{l}\right\rangle}{\langle c\rangle^{3}}\right) \mid D_{l 2} \cap D_{l 1} \in S_{l}\left(D_{l 2} \cap D_{l 1}, 1\right) \text { when } D_{l 2} \cap D_{l 1} \neq \emptyset .
\end{gathered}
$$

Since the support of any first order derivatives of $\chi_{\dot{s}}\left(10 / \varepsilon\left\langle g_{l}\right\rangle /\langle c\rangle^{3}\right)$ contained in $D_{l 1} \cap D_{l 2}$, boundedness of $\chi_{i}$ and (2.68) imply that 


$$
\left.\chi_{i}\left(\frac{10}{\varepsilon}\left\langle g_{l}\right\rangle \mid\langle c\rangle^{3}\right)\right|_{D_{l 2}} \in S_{l}\left(D_{l 2}, 1\right) \text { when } \quad D_{l 2} \neq \emptyset .
$$

From (2.66), (2.67), and (2.69) Lemma 2.4 implies that

$$
\left.\Psi_{i}^{(l, j)}\right|_{D_{l j}} \in S_{l}\left(D_{l j}, 1\right) \text { when } \quad D_{l j} \neq \emptyset
$$

Since supp $\Psi_{i}^{(l, j)} \subseteq D_{l_{j}}$, one obtains that

$$
\Psi_{i}^{(l, j)} \in S_{\phi_{l,}^{0}, \varphi_{l}}^{0,0} \text { for } j \neq 0 .
$$

We also get that $\Psi_{i}^{(l, 0)} \in S_{\Phi_{l}, \varphi_{l}}^{(l, 0)}$, because (2.65) holds with $N$ replaced by $N^{\prime}>1$ and supp $\Psi_{i}^{(l, 0)} \subseteq \Gamma_{l}$.

Now we can derive the estimates of derivatives of $\tilde{\Psi}_{i}^{(l, s, j)}$ and $\Lambda^{(l, s, j)}$. Noting the estimates (2.49), (2.58) and using Lemma 2.2 we see that $\varphi_{i 1_{j}}\left(w_{l}(\cdot)\right) \in$ $S_{l}\left(D_{l 1}, 1\right)$ when $D_{l 1} \neq \emptyset$. Using this, (2.71), and Lemma 2.4 we obtain that

$$
\tilde{\Psi}_{i}^{(l, 1, j)} \in S_{\Phi_{l}, \varphi_{l}}^{0,0} .
$$

Using similar argument for $\tilde{\Psi}_{i}^{(l, 2, j)}$ we get that $\tilde{\Psi}_{\Phi_{l}, \varphi_{l}}^{(l, 2, j)} \in S_{\Phi_{l}, \varphi_{l}}^{0,0}$.

Using (2.46) and Lemma 2.2 we see that $\mu_{1}\left(w_{l}(\cdot)\right)-1 \in S_{l}\left(D_{l 1}, \frac{\left.\left|g_{l}\right|\right|_{D_{l 1}}}{\left(|c| \mid D_{l 1}\right)^{3}}\right.$ when $D_{l 1} \neq \emptyset$. This implies that $\left.\Lambda_{1}^{(l, 1, j)}\right|_{D_{l 1}} \in S_{l}\left(D_{l 1}, \frac{\left.\left|g_{l}\right|\right|_{D_{l 1}}}{\left(\left.|c|\right|_{D_{l 1}}\right)^{2}}\right)$ when $D_{l 1} \neq \emptyset$. Noting supp $\Lambda_{1}^{(l, 1, j)} \subseteq D_{l 1}$ one obtains that $\Lambda_{1}^{(l, 1, j)} \in S_{\Phi_{l}, \varphi_{l}}^{(1,0)}$. Similarly, noting supp $\Lambda_{k}^{(l, 1, j)} \subseteq w_{l}^{-1}\left(U_{1 j}\right)$ when $D_{l 1} \neq \emptyset$ and using (2.55), we see that

$$
\left.\Lambda_{k}^{(l, 1, j)}\right|_{D_{l 1}} \in S_{l}\left(D_{l 1},\left.|c|\right|_{D_{l 1}}\left|w_{l 1}\right|^{3 / 2}\right) \text { for } k=2,3 \text { when } D_{l 1} \neq \emptyset .
$$

From $|c|\left|w_{l 1}\right|^{3 / 2}=\frac{\left|g_{l}\right|^{1 / 2}}{|c|^{1 / 2}}$ on $D_{l 1}$, this implies that $\Lambda_{k}^{(l, 1, j)} \in S_{\Phi_{l}, \varphi_{l}}^{1,0}$ for $k=2,3$. Similar argument also shows that $\Lambda_{k}^{(l, 2, j)} \in S_{\mathscr{\phi}_{l}, \varphi_{l}}^{1,0}$. Finally we shall derive other facts on $\tilde{\Psi}_{i}^{(l, 1, j)}$ and $\Lambda_{k}^{(l, 1, j)}, \tilde{\Psi}_{i}^{(l, 2, j)}$ and $\Lambda_{k}^{(l, 2, j)}, \Psi_{i}^{(l, 0)}$ respectively corresponding to (II), (I), and (III) in Lemma 2.3. First we consider (II).

Since $\Phi_{l}=\left\langle g_{l}\right\rangle^{1 / 3}$ on $\Gamma_{l}$, the definition of $D_{l 1}$ implies (iii) with $\psi_{0 k}$ replaced by $\tilde{\Psi}_{0}^{(l, 1, j)}$ when $\tilde{\Psi}_{0}^{(l, 1, j)} \neq 0$. (iv) and (v) with $\psi_{0 k}, \Lambda_{j}$ replaced by $\tilde{\Psi}_{0}^{(l, 1, j)}, \Lambda_{j}^{\left(l, 1, j^{\prime}\right)}$ follows from (2.43) and (2.44) when $\Psi_{0}^{\left(l, 1, j^{\prime}\right)} \neq 0$. (iv) with the same convention as before follows from (2.73) and (2.43). (i) follows from the fact that $p=p_{l}+b_{l 0},(2.1),(2.2)$, and that for $k=2,3$

$$
\begin{aligned}
& \left(\lambda_{l}+\Lambda_{k}^{(l, 1, j)}\right)\left(x, \xi^{\prime}\right)=\left[\tilde{\Psi}_{0}^{(l, 1, j)}\left(\lambda_{l}+\tilde{\Lambda}_{k}^{(l, 1, j)}\right)\right]\left(x, \xi^{\prime}\right)+\left[\left(1-\Psi_{0}^{(l, 1, j)}\right) \lambda_{l}\right]\left(x, \xi^{\prime}\right) \\
& \quad \text { on } \operatorname{supp} \Psi_{0}^{(l, 1, j)}
\end{aligned}
$$

where $\tilde{\Lambda}_{k}^{(l, 1, j)}\left(x, \xi^{\prime}\right)=\tilde{\mu}_{1 j k}\left(w_{l}\left(x, \xi^{\prime}\right)\right) c_{l}\left(x, \xi^{\prime}\right)$. (ii) follows from similar reason. 
Nest, with the convention that $\psi_{0 k}$ and $\Lambda_{j}$ replaced by $\tilde{\Psi}_{i}^{\left(l, 2, j^{\prime}\right)}$ and $\Lambda_{j}^{\left(l, 2, j^{\prime}\right)}$, (II) follows similarly. Finally (III) with $\psi_{0 k}$ replaced by $\tilde{\Psi}_{i}^{(l, 0)}$ follows from the definition of $\Phi_{l}$.

Step 5. Now we shall define $\psi_{i k}$. Set

$$
\tilde{\Psi}=\sum_{l=1}^{2} \sum_{s=1}^{2} \sum_{j=1}^{k_{s}} \tilde{\Psi}_{2}^{(l, s, j)}+\sum_{l=1}^{2} \Psi_{2}^{(l, 0)}
$$

Then $\tilde{\Psi} \geq 1$. We set

$$
\tilde{\tilde{\Psi}}^{(l, s, j)}=\tilde{\Psi}_{2}^{(l, s, j)} / \tilde{\Psi}, \tilde{\Psi}^{(l, 0)}=\Psi_{2}^{(l, 0)} / \tilde{\Psi}
$$

Since $\tilde{\Psi}^{-1} \in S_{1 / 2,1 / 2}^{0}\left(\mathbb{R}^{n} \times \boldsymbol{R}^{n-1}\right)$, and since $\Phi_{l} \leq C\left\langle\xi^{\prime}\right\rangle^{1 / 2}, \tilde{\Psi}^{(l, 0)} \in S_{\Phi_{l}, 0}^{0,0}$. Using the fact that $1 / 100 \leq \frac{\left\langle g_{2}\right\rangle}{\left\langle g_{1}\right\rangle} \leq 100$ on $D_{1} \cap D_{2}$ and that $\Phi_{l^{\prime}} \leq C\left\langle\xi^{\prime}\right\rangle^{1 / 2}$ on $\Gamma_{0 l}(N) \cap$ $D_{l^{\prime} j}$ when $l \neq l^{\prime}$, one can easily see $\tilde{\tilde{\Psi}}^{(l, s, j)} \in S_{\dot{\phi}_{l}, \varphi_{l}}^{0,0}$. Now we set

$$
\tilde{\psi}_{1 k}= \begin{cases}\tilde{\psi}_{1 k}=\tilde{\Psi}^{(k, 0)}(k=1,2) \\ \tilde{\tilde{\Psi}}^{\left(l, 2, k-\left(2+l k_{1}+(l-1) k_{2}\right)\right)} & \left(3+(l-1) k_{1}+(l-1) k_{2} \leq k \leq 2+l k_{1}+(l-1) k_{2}\right)\end{cases}
$$

and we define $\tilde{\psi}_{0 k}$ by the same definition as above with $\tilde{\Psi}^{(k, 0)}$ and $\tilde{\tilde{\Psi}}^{(\cdot, \cdot, \cdot)}$ replaced by $\Psi_{1}^{(k, 0)}$ and $\tilde{\Psi}^{(\cdot, \cdot, \cdot)}$ respectively. Then $\psi_{i k}$ is defined as follows:

$$
\psi_{i k}=\tilde{\psi}_{i j(k)}, \quad k=1, \cdots, k_{0}
$$

where $\left\{j(1), \cdots, j\left(k_{0}\right)\right\}=\left\{j ; \tilde{\psi}_{1 j} \neq 0\right\}$. This $\left\{\psi_{i k}\right\}$ has required properties from Step 4. The proof of Lemma 2.3 is complete.

\section{§3. Local Carleman Estimates}

Let $\Phi, \varphi \in C^{\infty}\left(\boldsymbol{R}^{n} \times \mathbb{R}^{n-1}\right)$ be a pair of weight functions stated after Lemma 2.2. Let $\psi_{i}(i=0,1) \in S_{\phi^{\prime}, \varphi}^{0,0}$ satisfying that

$$
\begin{gathered}
\psi_{1} \neq 0, \\
\psi_{0}=1 \text { on a neighbourhood of } \operatorname{supp} \psi_{1} .
\end{gathered}
$$

Let $P$ be a pseudodifferential operator on $\mathbb{R}^{n}$ with the symbol $p(x, \xi)$ given by

$$
p(x, \xi)=\left(\xi_{1}-\lambda\left(x, \xi^{\prime}\right)\right)^{2}\left(\xi_{1}-\lambda\left(x, \xi^{\prime}\right)-c\left(x, \xi^{\prime}\right)\right)+g\left(x, \xi^{\prime}\right)
$$

with

$$
\lambda, c \in S_{1,0}^{1}\left(\boldsymbol{R}^{n} \times \boldsymbol{R}^{n-1}\right), \quad g \in S_{1,0}^{3}\left(\boldsymbol{R}^{n} \times \boldsymbol{R}^{n-1}\right) \cap S_{\Phi, \varphi}^{3,0} .
$$

We assume that 


$$
C\left|\operatorname{Im} \lambda\left(x, \xi^{\prime}\right)\right| \geq\left\langle\xi^{\prime}\right\rangle, \quad C\left|\operatorname{Im}\left[(\lambda+c)\left(x, \xi^{\prime}\right)\right]\right| \geq\left\langle\xi^{\prime}\right\rangle
$$

for some positive constant $C$, and that one of the following (I), (II), (III) holds. (I) There exist $\Lambda_{i}(i=1,2,3) \in S_{\phi ; \varphi}^{1,0}$ such that

$$
\begin{gathered}
p(x, \xi)=\prod_{i=1}^{3}\left(\xi_{1}-\lambda\left(x, \xi^{\prime}\right)-\Lambda_{i}\left(x, \xi^{\prime}\right)\right) \text { when }\left(x, \xi^{\prime}\right) \in \operatorname{supp} \psi_{0}, \\
\sup _{\left(x, \xi^{\prime}\right) \in \text { supp } \psi_{0}}\left|c\left(x, \xi^{\prime}\right)\right| \Phi^{-1}\left(x, \xi^{\prime}\right)<+\infty, \\
\inf _{\left(x, \xi^{\prime}\right) \in \text { supp } \psi_{0}}\left|\Lambda_{i}\left(x, \xi^{\prime}\right)-\Lambda_{j}\left(x, \xi^{\prime}\right)\right| \Phi^{-1}\left(x, \xi^{\prime}\right)>0 \text { for any distinct } i, j, \\
\min _{1 \leq i \leq 3} \inf _{\left|\xi^{\prime}\right| \geq R}\left|\operatorname{Im}\left[\lambda\left(x, \xi^{\prime}\right)+\Lambda_{i}\left(x, \xi^{\prime}\right)\right]\right|\left\langle\xi^{\prime}\right\rangle^{-1}>0 \text { for some } R>0 .
\end{gathered}
$$

(II) There exist $\Lambda_{i}(i=1,2,3) \in S_{\varphi ; \varphi}^{1,0}$ such that

$$
p(x, \xi)=\left(\xi_{1}-\lambda\left(x, \xi^{\prime}\right)-c\left(x, \xi^{\prime}\right)-\Lambda_{1}\left(x, \xi^{\prime}\right)\right) \prod_{i=2}^{3}\left(\xi_{1}-\lambda\left(x, \xi^{\prime}\right)-\Lambda_{i}\left(x, \xi^{\prime}\right)\right)
$$

when $\left(x, \xi^{\prime}\right) \in \operatorname{supp} \psi_{0}$,

$$
\begin{gathered}
\inf _{\left(x, \xi^{\prime}\right) \in \text { supp } \psi_{0}}\left|c\left(x, \xi^{\prime}\right)\right| \Phi^{-1}\left(x, \xi^{\prime}\right)>0, \\
\inf _{\left(x, \xi^{\prime}\right) \in \text { supp } \psi_{0}}\left|c\left(x, \xi^{\prime}\right)+\Lambda_{1}\left(x, \xi^{\prime}\right)-\Lambda_{i}\left(x, \xi^{\prime}\right)\right|\left|c\left(x, \xi^{\prime}\right)\right|^{-1}>0 \text { for } i=2,3, \\
\Lambda_{i}\left(x, \xi^{\prime}\right)(i=2,3) \text { are distinct when }\left(x, \xi^{\prime}\right) \in \operatorname{supp} \psi_{0}, \\
\left|\partial_{x}^{\alpha} \partial_{\xi^{\prime}}^{\beta} \Lambda_{i}\left(x, \xi^{\prime}\right)\right| \leq C_{\alpha, \beta}\left|\Lambda_{2}\left(x, \xi^{\prime}\right)-\Lambda_{3}\left(x, \xi^{\prime}\right)\right| \Phi^{-|\beta|}\left(x, \xi^{\prime}\right) \varphi^{-|\alpha|}\left(x, \xi^{\prime}\right)
\end{gathered}
$$

on a neighbourhood of supp $\psi_{0}$ for $i=2,3$,

$$
\begin{gathered}
\left.\inf _{\left|\xi^{\prime}\right| \geq R}\left|\operatorname{Im}\left[\left(\lambda+c+\Lambda_{1}\right)\left(x, \xi^{\prime}\right)\right]\right|\left\langle\xi^{\prime}\right\rangle^{-1}\right\rangle 0 \text { and } \\
\left.\inf _{\left|\xi^{\prime}\right| \geq R}\left|\operatorname{Im}\left[\left(\lambda+\Lambda_{i}\right)\left(x, \xi^{\prime}\right)\right]\right|\left\langle\xi^{\prime}\right\rangle^{-1}\right\rangle 0 \text { for } i=2,3
\end{gathered}
$$

with some $R>0$.

(III) $\sup _{\left(x, \xi^{\prime}\right) \in \text { supp } \psi_{0}} \Phi\left(x, \xi^{\prime}\right)\left(1+\left|\xi^{\prime}\right|\right)^{-1 / 2}<+\infty$.

The main result of this section is the following proposition. This gives the estimates for $P$ in Proposition 1.1 on supports of functions $\psi_{j k}$ in Lemma 2.3. We set $\Psi_{i}=\psi_{i}\left(x, D^{\prime}\right)$.

Proposition 3.1. (1) Assume that (I) or (II) in the above holds. Then there exists positive constants $\tau_{0}, T_{0}, C_{0}$ such that

$$
\begin{aligned}
& T^{-1 / 2} A_{1}\left(\Psi_{1} u\right)+A_{2}\left(\Psi_{1} u\right)+A_{3}\left(\Psi_{1} u\right) \\
& \quad \leq C_{0}\left(\|P u\|_{T}^{(\tau)}+A_{1}(u)+T^{1 / 2} A_{3}(u)+T^{-1} R(u)\right)
\end{aligned}
$$


for $u \in \mathcal{S}_{T}\left(\mathbb{R}^{n}\right)$ when $\tau T^{2}>\tau_{0}$ and $T<T_{0}$.

Here

$$
\begin{aligned}
& A_{1}(u)=\sum_{1 \leq|\alpha|+|\beta| \leq 2}\left\|E_{(|\alpha|-|\beta|) / 2} P_{(\beta)}^{(\alpha)} u\right\|_{T}^{(\tau)}, \\
& A_{2}(u)=\|\| u\left\|_{T, 6}^{(\tau)}+T^{-1 / 2} \sum_{i \neq j}\right\| E_{\mathrm{y} / 2}\left(L_{\dot{i}} \circ L_{j}\right)(x, D) u \|_{T}^{(\tau)} \\
& +T^{-1} \sum_{i=1}^{3}\left\|E_{1} L_{i}(x, D) u\right\|_{T}^{(\tau)}, \\
& A_{3}(u)=T^{-1} \sum_{i=1}^{2}\left\|E_{1} L_{08}(x, D) u\right\|_{T}^{(\tau)}+T^{-1}\left\|E_{1} c\left(x, D^{\prime}\right) u\right\|_{T}^{(\tau)}, \\
& R(u)=\sum_{i=0}^{2}\left\|E_{-1 / 2+i} D_{1}^{2-i} u\right\|_{T}^{(\tau)}, \\
& L_{0 i}\left(x, \xi^{\prime}\right)= \begin{cases}\xi_{1}-\lambda\left(x, \xi^{\prime}\right) & (i=1) \\
\xi_{1}-\lambda\left(x, \xi^{\prime}\right)-c\left(x, \xi^{\prime}\right) & (i=2)\end{cases} \\
& L_{i}(x, \xi)=\xi_{1}-\lambda\left(x, \xi^{\prime}\right)-\Lambda_{i}\left(x, \xi^{\prime}\right) \text { except for that } \\
& L_{1}(x, \xi)=\xi_{1}-\lambda\left(x, \xi^{\prime}\right)-c\left(x, \xi^{\prime}\right)-\Lambda_{1}\left(x, \xi^{\prime}\right) \text { in case }(I I) \text {. }
\end{aligned}
$$

(2) Assume that (III) in the above holds. Then there exist positive constants $\tau_{0}, T_{0}, C_{0}$ such that

$$
T^{-1 / 2} A_{1}\left(\Psi_{1} u\right)+B\left(\Psi_{1} u\right) \leq C_{0}\left(\|P u\|_{T}^{(\tau)}+A_{1}(u)+T^{-1 / 2} R(u)\right)
$$

for $u \in \mathcal{S}_{T}\left(\boldsymbol{R}^{n}\right)$ when $\tau T^{2}>\tau_{0}$ and $T<T_{0}$.

Here

$$
\begin{aligned}
B(u)= & \|\| u\left\|_{T, 6}^{(\tau)}+\sum_{i \neq j} \tau^{-1}\right\|\left|\left(L_{0 i} \circ L_{0 j}\right)(x, D) u\left\|_{T, 2}^{(\tau)}+\sum_{i=1}^{2} \tau^{-1 / 2} \mid\right\| L_{0 i}(x, D) u \|_{T, 4}^{(\tau)}\right. \\
& +\tau^{-1 / 2}\|\| c\left(x, D^{\prime}\right) u\|\|_{T, 4}^{(\tau)}+\tau^{-1}\left\|\mid L_{01}(x, D)^{2} u\right\| \|_{T, 2}^{(\tau)},
\end{aligned}
$$

and the other notations are the same as in (1).

We shall prove this proposition in this section admitting one proposition and several lemmas which will be proved in later sections. We first prepare a proposition of Carleman estimate for first order factors in the factorizations of $p$ and $p-g$ having the basic role for proof of Proposition 3.1.

Proposition 3.2. (1) Let $L(x, \xi)=\xi_{1}-a\left(x, \xi^{\prime}\right)-b\left(x, \xi^{\prime}\right)$ with

$$
\begin{gathered}
a \in S_{1,0}^{1}\left(\mathbb{R}^{n} \times \mathbb{R}^{n-1}\right), \quad b \in S_{\mathscr{\phi}, \varphi}^{1,0}, \\
\inf _{\left|\xi^{\prime}\right| \geq R}\left|\operatorname{Im}\left[(a+b)\left(x, \xi^{\prime}\right)\right]\right|\left\langle\xi^{\prime}\right\rangle^{-1}>0 \text { for some } R>0 .
\end{gathered}
$$


Then there exist positive constants $\tau_{0}, T_{0}, C_{0}$ such that

$$
\tau^{-1}\|u\|\left\|_{T, 2}^{(\tau)} \leq C_{0}\right\| L(x, D) u \|_{T}^{(\tau)}, \quad u \in \mathcal{S}_{T}\left(\boldsymbol{R}^{n}\right)
$$

when $\tau T^{2}>\tau_{0}$ and $T<T_{0}$.

(2) Let $L_{i}(x, \xi)(i=1,2,3)$ be given by $L_{i}(x, \xi)=\xi_{1}-a_{i}\left(x, \xi^{\prime}\right)-b_{i}\left(x, \xi^{\prime}\right)$ with $a_{i} \in S_{1,0}^{1}\left(\boldsymbol{R}^{n} \times \boldsymbol{R}^{n-1}\right), b_{i} \in S_{\phi^{\prime}, \varphi}^{1,0}$ satisfying (3.3) with $a, b$ replaced by $a_{i}, b_{i}$ respectively. Then there exist positive constants $\tau_{0}, T_{0}, C_{0}$ such that

$$
\begin{aligned}
& \tau^{-1 / 2}\|u\|\left\|_{T, 4}^{(\tau)} \leq C_{0}\right\|\left(L_{i} \circ L_{j}\right)(x, D) u \|_{T}^{(\tau)}, \quad u \in \mathcal{S}_{T}\left(\boldsymbol{R}^{n}\right) \text { for any } i, j, \\
& \|u\|_{T, 6}^{(\tau)} \leq C_{0} \sum_{\sigma \in S_{3}}\left\|\left(L_{\sigma(1)} \circ L_{\sigma(2)} \circ L_{\sigma(3)}\right)(x, D) u\right\|_{T}^{(\tau)}, \quad u \in \mathcal{S}_{T}\left(\boldsymbol{R}^{n}\right)
\end{aligned}
$$

when $\tau T^{2}>\tau_{0}$ and $T<T_{0}$. Here $S_{3}$ is the symmetric group of degree 3 .

Next we prepare some lemmas which need for the proof of both of (1) and (2) in Proposition 3.1.

Lemma 3.1. Let $a \in S_{1 / 2,1 / 2}^{m}\left(\boldsymbol{R}^{n} \times \boldsymbol{R}^{n-1}\right)$. Then there exist positive constant $C$ such that for any $\tau$ and $T$,

$$
\left\|a\left(x, D^{\prime}\right) u\right\|_{T}^{(\tau)} \leq C\left\|E_{m} u\right\|_{T}^{(\tau)}, \quad u \in \mathcal{S}\left(\boldsymbol{R}^{n}\right) .
$$

Next two lemmas give estimates for commutators.

Lemma 3.2. Let $L_{i}(x, \xi)(i=1,2,3)$ be the same as in Proposition 3.2-(2). Then there exist positive constants $\tau_{0}, T_{0}, C_{0}$ such that $C_{0}^{-1}\left\|\left(L_{1} \circ L_{2} \circ L_{3}\right)(x, D) u\right\|_{T}^{(\tau)} \leq\left\|\left(L_{1} L_{2} L_{3}\right)(x, D) u\right\|_{T}^{(\tau)} \leq C_{0}\left\|\left(L_{1} \circ L_{2} \circ L_{3}\right)(x, D) u\right\| \|_{T}^{(\tau)}$ for $u \in \mathcal{S}_{T}\left(\boldsymbol{R}^{n}\right)$ when $\tau T^{2}>\tau_{0}$ and $T<T_{0}$.

Lemma 3.3. Let $\chi \in S_{\Phi, \varphi}^{0,0}$. Then we have that

$$
p \circ \chi-\chi \circ p=\sum_{1 \leq|\alpha|+|\beta| \leq 2} a_{\alpha \beta} \circ p_{(\beta)}^{(\alpha)}+\sum_{j=0}^{2} b_{j} \xi_{1}^{2-j}
$$

with some $a_{\alpha \beta} \in S_{\bar{\phi},, \varphi}^{-|\alpha|,-|\beta|}$ and $b_{j} \in S_{1 / 2,1 / 2}^{-(1 / 2)+j}\left(\boldsymbol{R}^{n} \times \boldsymbol{R}^{n-1}\right)$.

Lemma 3.4. Let $\chi \in S_{\Phi, \varphi}^{0,0}$. Then we have that for $b \in S_{1 / 2,1 / 2}^{-(1 / 2)+j}\left(\boldsymbol{R}^{n} \times \boldsymbol{R}^{n-1}\right)$ with $j=0,1,2$

$$
\left(b \xi_{1}^{2-j}\right) \circ \chi=\sum_{k=j}^{2} a_{k} \xi_{1}^{2-k} \quad \text { with some } \quad a_{k} \in S_{1 / 2,1 / 2}^{-(1 / 2)+((j+k) / 2)}\left(\boldsymbol{R}^{n} \times \boldsymbol{R}^{n-1}\right) .
$$

Next two lemmas are ones for handling neglizible terms.

Lemma 3.5. Let $\chi \in S_{\emptyset, \varphi}^{-3,-3}$. Then there exist positive constants $\tau_{0}, T_{0}, C_{0}$ 
such that when $\tau T^{2}>\tau_{0}$ and $T<T_{0}$.

$$
B\left(\chi\left(x, D^{\prime}\right) u\right)+\left\|g\left(x, D^{\prime}\right) \chi\left(x, D^{\prime}\right) u\right\|_{T}^{(\tau)} \leq C_{0}\left(\|P u\|_{T}^{(\tau)}+A_{1}(u)+R(u)\right)
$$

for $u \in \mathcal{S}_{T}\left(\boldsymbol{R}^{n}\right)$.

Lemma 3.6. Let $\chi \in S_{\Phi, \varphi}^{-3,-3}$. Then there exist positive constants $\tau_{0}, T_{0}, C_{0}$ such that when $\tau T^{2}>\tau_{0}$ and $T<T_{0}$,

$$
\begin{aligned}
& T^{-1 / 2} A_{1}\left(\chi\left(x, D^{\prime}\right) u\right)+T^{-1 / 2} \sum_{1 \leq|\alpha|+\mid \beta_{\mid \leq 2}}\left\|E_{(|\alpha|-|\beta|) / 2} g_{(\beta)}^{(\alpha)}\left(x, D^{\prime}\right) \chi\left(x, D^{\prime}\right) u\right\|_{T}^{(\tau)} \\
& \quad \leq C_{0}\left(\|P u\|_{T}^{(\tau)}+A_{1}(u)+T^{-1 / 2} R(u)\right)
\end{aligned}
$$

for $u \in \mathcal{S}_{T}\left(\boldsymbol{R}^{n}\right)$

Now we start to prove Proposition 3.1.

Proof of (1). First we estimate $A_{2}\left(\Psi_{1} u\right)$. We break up into $\Psi_{0} \Psi_{1}$ and $\left(1-\Psi_{0}\right) \Psi_{1}$. Then

$$
A_{2}\left(\Psi_{1} u\right) \leq A_{2}\left(\Psi_{0} \Psi_{1} u\right)+A_{2}\left(\left(1-\Psi_{0}\right) \Psi_{1} u\right)
$$

We take up the first term on the right hand side first. From Proposition 3.2 and Lemma 3.2 there exist positive constants $\tau_{1}, T_{1}, C_{1}$ such that when $\tau T^{2}>\tau_{1}$ and $T<T_{1}$,

$$
A_{2}(u) \leq C_{1}\left\|\left(L_{1} L_{2} L_{3}\right)(x, D) u\right\|_{T}^{(\tau)}, \quad u \in \mathcal{S}_{T}\left(\mathbb{R}^{n}\right)
$$

We need a lemma to estimate $\left(L_{1} L_{2} L_{3}\right)(x, D) \Psi_{0}-\Psi_{0} P$.

Lemma 3.7. Assume that $(I)$ or $(I I)$ holds. Then if $\chi \in S_{\phi, \varphi}^{0,0}$ with supp $\chi \subseteq$ $\operatorname{supp} \psi_{0}$,

$$
\begin{aligned}
\left(L_{1} L_{2} L_{3}\right) \circ \chi-\chi \circ p & =\sum_{i \neq j} a_{i j} \circ L_{i} \circ L_{j}+\sum_{i=1}^{3} a_{i} \circ L_{i} \\
& +a_{0}+\sum_{1 \leq|\alpha|+|\beta| \leq 2} a_{\alpha \beta} \circ p_{(\beta)}^{(\alpha)}+\sum_{i=0}^{2} b_{i} \xi_{1}^{2-i}
\end{aligned}
$$

with $\quad a_{i j} \in S_{\phi ;,}^{0,-1}, \quad a_{i} \in S_{\phi, \varphi}^{0,-2} \quad$ for $\quad i \neq 0, \quad a_{0} \in S_{\phi, \varphi}^{1,-2}, \quad a_{\alpha \beta} \in S_{\bar{\phi}, \varphi}^{-|\beta|,-|\alpha|}, \quad b_{i} \in$ $S_{1 / 2,1 / 2}^{-(1 / 2)+i}\left(\mathbb{R}^{n} \times \mathbb{R}^{n-1}\right)$.

We note that

$$
S_{\Phi, \varphi}^{M, m} \subseteq\left\{\begin{array}{ll}
S_{1 / 2,1 / 2}^{(M-m) / 2}\left(\mathbb{R}^{n} \times \boldsymbol{R}^{n-1}\right) & (-m \geq M \geq 0) \\
S_{1 / 2,1 / 2}^{-(M+m) / 2}\left(\boldsymbol{R}^{n} \times \boldsymbol{R}^{n-1}\right) & (m \leq 0, M \leq 0)
\end{array} .\right.
$$


Substituting $\Psi_{0} u$ to $u$ in (3.5), using Lemma 3.7 with $\chi=\psi_{0}$, and noting (3.6) we obtain the following inequality with the notations in Lemma 3.7: there exists a positive constant $C_{2}$ such that when $\tau T^{2}>\tau_{1}$ and $T<T_{1}$, for $u \in \mathcal{S}_{T}\left(\boldsymbol{R}^{n}\right)$

$$
\begin{aligned}
A_{2}\left(\Psi_{0} u\right) \leq & C_{1}\left(\left\|\Psi_{0} P u\right\|_{T}^{(\tau)}+\sum_{i \neq j}\left\|a_{i j}\left(x, D^{\prime}\right)\left(L_{i} \circ L_{j}\right)(x, D) u\right\|_{T}^{(\tau)}\right. \\
& +\sum_{i=1}^{3}\left\|a_{i}\left(x, D^{\prime}\right) L_{i}(x, D) u\right\|_{T}^{(\tau)}+\left\|a_{0}\left(x, D^{\prime}\right) u\right\|_{T}^{(\tau)} \\
& \left.+\sum_{1 \leq|\alpha|+|\beta| \leq 2}\left\|a_{\alpha \beta}\left(x, D^{\prime}\right) P_{(\beta)}^{(\alpha)} u\right\|_{T}^{(\tau)}+\sum_{i=0}^{2}\left\|b_{i}\left(x, D^{\prime}\right) D_{1}^{2-i} u\right\|_{T}^{(\tau)}\right) \\
\leq & C_{2}\left(\|P u\|_{T}^{(\tau)}+\sum_{i \neq j}\left\|E_{1 / 2}\left(L_{i} \circ L_{j}\right)(x, D) u\right\|_{T}^{(\tau)}\right. \\
& +\sum_{i=1}^{3}\left\|E_{1} L_{i}(x, D) u\right\|_{T}^{(\tau)}+\left\|E_{3 / 2} u\right\|_{T}^{(\tau)} \\
& \left.+\sum_{1 \leq|\alpha|+|\beta| \leq 2}\left\|E_{(|\alpha|-|\beta|) / 2} P_{(\beta)}^{(\alpha)} u\right\|_{T}^{(\tau)}+R(u)\right) \\
\leq & C_{2}\left(\|P u\|_{T}^{(\tau)}+\left(T^{1 / 2}+T\right) A_{2}(u)+A_{1}(u)+2 R(u)\right)
\end{aligned}
$$

From Lemma 3.4 there exists a positive constant $C_{3}$ such that for any $\tau, T$

$$
R\left(\Psi_{1} u\right) \leq C_{3} R(u), u \in \mathcal{S}\left(\boldsymbol{R}^{n}\right)
$$

From Lemma 3.3 there exists a positive constant $C_{4}$ such that for any $\tau, T$

$$
\left\|P \Psi_{1} u\right\|_{T}^{(\tau)} \leq C_{4}\left(\|P u\|_{T}^{(\tau)}+A_{1}(u)+R(u)\right), \quad u \in \mathcal{S}_{T}\left(\boldsymbol{R}^{n}\right) .
$$

Substituting $\Psi_{1} u$ into $u$ in (3.7) and using (3.8) and (3.9) we obtain that when $\tau T^{2}>\tau_{1}$ and $T<T_{1}$, for $u \in \mathcal{S}_{T}\left(\boldsymbol{R}^{n}\right)$

$$
\begin{aligned}
A_{2}\left(\Psi_{0} \Psi_{1} u\right) \leq & C_{2} C_{4}\left(\|P u\|_{T}^{(\tau)}+A_{1}(u)\right)+C_{2}\left(C_{4}+2 C_{3}\right) R(u) \\
& +C_{2}\left(T^{1 / 2}+T\right) A_{2}\left(\Psi_{1} u\right)+C_{2} A_{1}\left(\Psi_{1} u\right) .
\end{aligned}
$$

Next we handle the second term on the right hand side of (3.4).

Lemma 3.8. Let $\chi \in S_{\Phi, \dot{\varphi}}^{-3,-3}$.

(1) Assume that (I) holds.

(i) If $i \neq j$,

$$
\left\langle\xi^{\prime}\right\rangle^{1 / 2} \circ L_{i} \circ L_{j} \circ \chi=\left\langle\xi^{\prime}\right\rangle^{1 / 2} \circ L_{01} \circ L_{01} \circ \chi+a_{i j} \circ L_{01}+a_{i j}^{\prime}
$$

with $a_{i j} \in S_{\Phi, \varphi}^{0,-2}, a_{i j}^{\prime} \in S_{\Phi, \varphi}^{0,-3}$.

(ii) $\left\langle\xi^{\prime}\right\rangle \circ L_{i} \circ \chi=\left\langle\xi^{\prime}\right\rangle \circ L_{01} \circ \chi+a_{i}$

with $a_{i} \in S_{\varphi, \varphi}^{0,-3}$.

(2) Assume that (II) holds.

(i) If $i \neq j$ and $i \neq 1, j \neq 1$, (i) in (1) holds. If $i \neq j$ and one of $i$ and $j$ is equal 
to 1 ,

$$
\left\langle\xi^{\prime}\right\rangle^{1 / 2} \circ L_{i} \circ L_{j} \circ \chi=\left\langle\xi^{\prime}\right\rangle^{1 / 2} \circ L_{01} \circ L_{02} \circ \chi+\sum_{k=1}^{2} a_{i j k} \circ L_{0 k}+a_{i j}^{\prime}
$$

with $a_{i j k} \in S_{\bar{\phi}, \varphi^{2}}^{0}, a_{i j}^{\prime} \in S_{\phi_{,},-3}^{0}$.

(ii) If $i \neq 1$, (ii) in (1) holds and we have

$$
\left\langle\xi^{\prime}\right\rangle \circ L_{1} \circ \chi=\left\langle\xi^{\prime}\right\rangle \circ L_{02} \circ \chi+a_{1}
$$

with $a_{1} \in S_{\Phi, \varphi}^{0,-3}$.

Lemma 3.8 easily implies the following.

Corollary 3.1. Assume (I) or (II) holds. Let $\chi \in S_{\Phi, \phi}^{-3,-3}$. Then there exists a positive constant $C_{0}$ such that for any $\tau, T$, and $u \in S\left(\mathbb{R}^{n}\right)$.

$$
\begin{aligned}
\sum_{i \neq j} & \left\|E_{1 / 2}\left(L_{\dot{i}} \circ L_{j}\right)(x, D) \chi\left(x, D^{\prime}\right) u\right\|_{T}^{(\tau)} \\
\quad \leq & C_{0}\left(\sum_{k=1}^{2}\left\|E_{1 / 2}\left(L_{01} \circ L_{0 k}\right)(x, D) \chi\left(x, D^{\prime}\right) u\right\|_{T}^{(\tau)}+\sum_{k=1}^{2}\left\|E_{1} L_{0 k}(x, D) u\right\|_{T}^{(\tau)}\right. \\
\quad & \left.\quad+\left\|E_{3 / 2} u\right\|_{T}^{(\tau)}\right), \\
\sum_{i=1}^{2}\left\|E_{1} L_{i}(x, D) \chi\left(x, D^{\prime}\right) u\right\|_{T}^{(\tau)} & \\
\quad \leq & C_{0}\left(\sum_{k=1}^{2}\left\|E_{1} L_{0 k}(x, D) \chi\left(x, D^{\prime}\right) u\right\|_{T}^{(\tau)}+\left\|E_{3 / 2} u\right\|_{T}^{(\tau)}\right) .
\end{aligned}
$$

From the fact that $\psi_{0}=1$ on a neighbourhood of supp $\psi_{1}$ we have

$$
\left(I-\Psi_{0}\right) \Psi_{1} \in O p S_{\bar{\phi}, \varphi}^{-N,-N} \text { for any } N>0 .
$$

Noting this and using Corollary 3.1 we see that there exists a positive constant $C_{5}$ such that for any $\tau, T$, and $u \in S\left(\mathbb{R}^{n}\right)$

$$
\begin{aligned}
T^{-1 / 2} & \sum_{i \neq j}\left\|E_{1 / 2}\left(L_{i} \circ L_{j}\right)(x, D)\left(I-\Psi_{0}\right) \Psi_{1} u\right\|_{T}^{(\tau)}+T^{-1} \sum_{k=1}^{3}\left\|E_{1} L_{k}(x, D)\left(I-\Psi_{0}\right) \Psi_{1} u\right\|_{T}^{(\tau)} \\
\leq & C_{5}\left(T^{-1 / 2} \sum_{k=1}^{2}\left\|E_{1 / 2}\left(L_{01} \circ L_{0 k}\right)(x, D)\left(I-\Psi_{0}\right) \Psi_{1} u\right\|_{T}^{(\tau)}+T^{1 / 2} A_{3}(u)+T^{-1 / 2} R(u)\right. \\
& \left.+T^{-1} \sum_{k=1}^{2}\left\|E_{1} L_{0 k}(x, D)\left(I-\Psi_{0}\right) \Psi_{1} u\right\|_{T}^{(\tau)}+T^{-1} R(u)\right) .
\end{aligned}
$$

Using this we obtain that for any $\tau, T$, and $u \in S\left(\mathbb{R}^{n}\right)$

$$
\begin{aligned}
A_{2}\left(\left(I-\Psi_{0}\right) \Psi_{1} u\right) \leq & \max \left(C_{5}, 1\right) B\left(\left(I-\Psi_{0}\right) \Psi_{1} u\right) \\
& +C_{5} T^{1 / 2} A_{3}(u)+C_{5}\left(T^{1 / 2}+1\right) T^{-1} R(u) .
\end{aligned}
$$

From Lemma 3.5 there exist positive constants $\tau_{2}>\tau_{1}$ and $T_{2}<T_{1}$ and $C_{6}$ such 
that

$$
B\left(\left(I-\Psi_{0}\right) \Psi_{1} u\right) \leq C_{6}\left(\|P u\|_{T}^{(\tau)}+A_{1}(u)+R(u)\right), \quad u \in \mathcal{S}_{T}\left(\boldsymbol{R}^{n}\right)
$$

when $\tau T^{2}>\tau_{2}$ and $T<T_{2}$.

Substituting this inequality to the above one we get that when $\tau T^{2}>\tau_{2}$ and $T<T_{2}$, for $u \in \mathcal{S}_{T}\left(\boldsymbol{R}^{n}\right)$

$$
\begin{aligned}
& A_{2}\left(\left(I-\Psi_{0}\right) \Psi_{1} u\right) \leq \max \left(C_{5}, 1\right) C_{6}\left(\|P u\|_{T}^{(\tau)}+A_{1}(u)\right)+C_{5} T^{1 / 2} A_{3}(u) \\
& \quad+\left\{\max \left(C_{5}, 1\right) C_{6} T+C_{5}\left(T^{1 / 2}+1\right)\right\} T^{-1} R(u) .
\end{aligned}
$$

From (3.4), (3.10), and (3.12) we obtain that when $\tau T^{2}>\tau_{2}$ and $T<T_{2}$, for $u \in \mathcal{S}\left(\boldsymbol{R}^{n}\right)$

$$
\begin{aligned}
A_{2}\left(\Psi_{1} u\right) \leq & C_{7}\left(\|P u\|_{T}^{(\tau)}+A_{1}(u)\right)+C_{8, T} T^{-1} R(u)+C_{5} T^{1 / 2} A_{3}(u) \\
& +C_{2}\left(T^{1 / 2}+T\right) A_{2}\left(\Psi_{1} u\right)+C_{2} A_{1}\left(\Psi_{1} u\right) .
\end{aligned}
$$

Here

$$
\begin{aligned}
& C_{7}=C_{2} C_{4}+\max \left(C_{5}, 1\right) C_{6}, \\
& C_{8, T}=\left\{C_{2}\left(C_{4}+2 C_{3}\right)+\max \left(C_{5}, 1\right) C_{6}\right\} T+C_{6}\left(T^{1 / 2}+1\right) .
\end{aligned}
$$

Next we estimate $A_{3}\left(\Psi_{1} u\right)$.

Lemma 3.9. (1) Assume that (I) holds. Then for any distinct $1 \leq i, j \leq 3$

$$
L_{01} \circ \psi_{1}=a_{1} \circ L_{i} \circ \psi_{1}+a_{2} \circ L_{j} \circ \psi_{1}+a_{3}+L_{01} \circ\left(1-\psi_{0}\right) \circ \psi_{1}
$$

with $a_{1}, a_{2} \in S_{\Phi, \varphi}^{0,0}$ and $a_{3} \in S_{\Phi, \varphi}^{0,-1}$. And for any distinct $1 \leq i, j \leq 3$

$$
c \circ \psi_{1}=a_{1} \circ L_{i} \circ \psi_{1}+a_{2} \circ L_{j} \circ \psi_{1}+a_{3}+c \circ\left(1-\psi_{0}\right) \circ \psi_{1}
$$

with $a_{1}, a_{2} \in S_{\phi, \varphi}^{0,0}$ and $a_{3} \in S_{\phi, \varphi}^{0,-1}$.

(2) Assume that (II) holds. Then for any distinct $1 \leq i, j \leq 3$, (3.15) holds. And for any $i \neq 1$

$$
c \circ \psi_{1}=a_{1} \circ L_{1} \circ \psi_{1}+a_{2} \circ L_{i} \circ \psi_{1}+a_{3}+c \circ\left(1-\psi_{0}\right) \circ \psi_{1}
$$

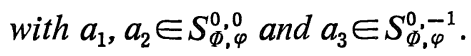

From Lemma 3.9 we see that there exist positive constants $C_{10}, C_{11}$ such that for any $\tau, T$, and $u \in \mathcal{S}\left(\boldsymbol{R}^{n}\right)$

$$
\begin{aligned}
\left\|E_{1} L_{01}(x, D) \Psi_{1} u\right\|_{T}^{(\tau)} \leq C_{9}( & \sum_{k=1}^{2}\left\|E_{1} L_{k}(x, D) \Psi_{1} u\right\|_{T}^{(\tau)}+\left\|E_{3 / 2} u\right\|_{T}^{(\tau)} \\
& \left.+\left\|E_{1} L_{01}(x, D)\left(I-\Psi_{0}\right) \Psi_{1} u\right\|_{T}^{(\tau)}\right)
\end{aligned}
$$




$$
\begin{gathered}
\left\|E_{1} c\left(x, D^{\prime}\right) \Psi_{1} u\right\|_{T}^{(\tau)} \leq C_{10}\left(\sum_{k=1}^{2}\left\|E_{1} L_{k}(x, D) \Psi_{1} u\right\|_{T}^{(\tau)}+\left\|E_{3 / 2} u\right\|_{T}^{(\tau)}\right. \\
\left.+\left\|E_{1} c\left(x, D^{\prime}\right)\left(I-\Psi_{0}\right) \Psi_{1} u\right\|_{T}^{(\tau)}\right)
\end{gathered}
$$

We obtain from these two inequalities that for any $\tau, T$, and $u \in \mathcal{S}\left(\mathbb{R}^{n}\right)$

$$
\begin{aligned}
A_{3}\left(\Psi_{1} u\right)= & T^{-1}\left(\left\|E_{1} L_{01}(x, D) \Psi_{1} u\right\|_{T}^{(\tau)}+\left\|E_{1}\left(L_{01}(x, D)-c\left(x, D^{\prime}\right)\right) \Psi_{1} u\right\|_{T}^{(\tau)}\right. \\
& \left.+\left\|E_{1} c\left(x, D^{\prime}\right) \Psi_{1} u\right\|_{T}^{(\tau)}\right) \\
\leq & 2 T^{-1}\left(\left\|E_{1} L_{01}(x, D) \Psi_{1} u\right\|_{T}^{(\tau)}+\left\|E_{1} c\left(x, D^{\prime}\right) \Psi_{1} u\right\|_{T}^{(\tau)}\right) \\
\leq & 2\left\{\left(C_{9}+C_{10}\right)\left(\sum_{k=1}^{2} T^{-1}\left\|E_{1} L_{k}(x, D) \Psi_{1} u\right\|_{T}^{(\tau)}+T^{-1}\left\|E_{3 / 2} u\right\|_{T}^{(\tau)}\right)\right. \\
& +C_{9} T^{-1}\left\|E_{1} L_{01}(x, D)\left(I-\Psi_{0}\right) \Psi_{1} u\right\|_{T}^{(\tau)} \\
& \left.+C_{10} T^{-1}\left\|E_{1} c\left(x, D^{\prime}\right)\left(I-\Psi_{0}\right) \Psi_{1} u\right\|_{T}^{(\tau)}\right\} \\
\leq & 2\left\{\left(C_{9}+C_{10}\right)\left(A_{2}\left(\Psi_{1} u\right)+T^{-1} R(u)\right)\right. \\
& \left.+\max \left(C_{9}, C_{10}\right) B\left(\left(I-\Psi_{0}\right) \Psi_{1} u\right)\right\}
\end{aligned}
$$

Substituting (3.13) and (3.11) into (3.16) we see that there exists a positive constant $C_{11}$ such that when $\tau T^{2}>\tau_{2}, T<T_{2}$, and $u \in \mathcal{S}_{T}\left(\mathbb{R}^{n}\right)$,

$$
\begin{aligned}
A_{3}\left(\Psi_{1} u\right) \leq & C_{11}\left(\|P u\|_{T}^{(\tau)}+A_{1}(u)+T^{1 / 2} A_{3}(u)+\left(T^{1 / 2}+T\right) A_{2}\left(\Psi_{1} u\right)\right. \\
& \left.+A_{1}\left(\Psi_{1} u\right)\right)+C_{12, T} T^{-1} R(u) .
\end{aligned}
$$

Here

$$
C_{12, T}=2\left\{C_{8, T}+C_{9}+C_{10}+\max \left(C_{9}, C_{10}\right) C_{6} T\right\} .
$$

Finally we estimate $A_{1}\left(\Psi_{1} u\right)$. First we have

$$
\left.A_{1}\left(\Psi_{1} u\right) \leq A_{1}\left(I-\Psi_{0}^{2}\right) \Psi_{1} u\right)+A_{1}\left(\Psi_{0}^{2} \Psi_{1} u\right), \quad u \in \mathcal{S}\left(\boldsymbol{R}^{n}\right)
$$

To estimate the second term on the right we need

Lemma 3.10. Let $\chi \in S_{\mathscr{\phi}, \varphi}^{0,0}$ with supp $\chi \subseteq \operatorname{supp} \psi_{0}$. Then if $\alpha, \beta \in \mathbb{Z}_{+}^{n}$ with $|\alpha|+|\beta|=1$ or 2 ,

$$
\left\langle\xi^{\prime}\right\rangle^{(|\alpha|-\mid \beta /) / 2} \circ p_{(\beta)}^{(\alpha)} \circ \chi=\sum_{i \neq j} a_{i j}^{\alpha \beta} \circ L_{i} \circ L_{j}+\sum_{i=1}^{3} a_{i}^{\alpha \beta} \circ L_{i}+\sum_{j=0}^{2} b_{j}^{\alpha \beta} \xi_{1}^{2-j}
$$

with $a_{i j}^{\alpha \beta} \in S_{1 / 2,1 / 2}^{1 / 2}\left(\boldsymbol{R}^{n} \times \boldsymbol{R}^{n-1}\right), a_{i}^{\alpha \beta} \in S_{1 / 2,1 / 2}^{1}\left(\boldsymbol{R}^{n} \times \boldsymbol{R}^{n-1}\right), b_{j}^{\alpha \beta} \in S_{1 / 2,1 / 2}^{-(1 / 2)+j}\left(\boldsymbol{R}^{n} \times \boldsymbol{R}^{n-1}\right)$.

From Lemma 3.10 we see that there exists a positive constant $C_{13}$ such that for any $\tau, T$, and $u \in S\left(\boldsymbol{R}^{n}\right)$

$$
T^{-1 / 2} A_{1}\left(\Psi_{0} u\right) \leq C_{13}\left(\sum_{i \neq j} T^{-1 / 2}\left\|E_{1 / 2}\left(L_{i} \circ L_{j}\right)(x, D) u\right\|_{T}^{(\tau)}\right.
$$




$$
\begin{aligned}
& \left.+T^{-1 / 2} \sum_{i=1}^{3}\left\|E_{1} L_{i}(x, D) u\right\|_{T}^{(\tau)}+T^{-1 / 2} R(u)\right) \\
\leq & C_{13}\left(\max \left(1, T^{1 / 2}\right) A_{2}(u)+T^{-1 / 2} R(u)\right) .
\end{aligned}
$$

Since $\Psi_{0} \Psi_{1} \in O p S_{\phi, \varphi}^{0,0}$, from Lemma 3.4 there exists a positive constant $C_{14}$ such that for any $\tau, T$, and $u \in \mathcal{S}\left(\boldsymbol{R}^{n}\right)$

$$
R\left(\Psi_{0} \Psi_{1} u\right) \leq C_{14} R(u)
$$

Substituting $\Psi_{0} \Psi_{1} u$ into $u$ in (3.20) and using (3.21) we get that for any $\tau, T$, and $u \in \mathcal{S}\left(\boldsymbol{R}^{n}\right)$

$$
T^{-1 / 2} A_{1}\left(\Psi_{0}^{2} \Psi_{1} u\right) \leq C_{13}\left(\max \left(1, T^{1 / 2}\right) A_{2}\left(\Psi_{0} \Psi_{1} u\right)+C_{14} T^{-1 / 2} R(u)\right)
$$

Substituting (3.10) into this inequality we see that there exists a positive constant $C_{15}$ such that when $\tau T^{2}>\tau_{1}$ and $T<T_{1}$,

$$
\begin{aligned}
& T^{-1 / 2} A_{1}\left(\Psi_{0}^{2} \Psi_{1} u\right) \leq C_{15} \max \left(1, T^{1 / 2}\right)\left(\|P u\|_{T}^{(\tau)}+A_{1}(u)+\left(T^{1 / 2}+T\right) A_{2}\left(\Psi_{1} u\right)\right. \\
& \left.\quad+A_{1}\left(\Psi_{1} u\right)+\left(T^{1 / 2}+T\right) T^{-1} R(u)\right), \quad u \in \mathcal{S}_{T}\left(\boldsymbol{R}^{n}\right) .
\end{aligned}
$$

Since $\left(I-\Psi_{0}^{2}\right) \Psi_{1}=\left(I+\Psi_{0}\right)\left(I-\Psi_{0}\right) \Psi_{1}$, and since $\left(I-\Psi_{0}\right) \Psi_{1} \in O p S_{\Phi, \varphi^{-N}}^{-N}$ for any $N>0$, we have

$$
\left(I-\Psi_{0}^{2}\right) \Psi_{1} \in O p S_{\Phi, \varphi}^{-N,-N} \text { for any } \quad N>0 .
$$

Thus from Lemma 3.6 there exist positive constants $\tau_{3}>\tau_{2}$ and $T_{3}<T_{2}$ and $C_{16}$ such that when $\tau T^{2}>\tau_{3}$ and $T<T_{3}$, for $u \in \mathcal{S}_{T}\left(\boldsymbol{R}^{n}\right)$

$$
T^{-1 / 2} A_{1}\left(\left(I-\Psi_{0}^{2}\right) \Psi_{1} u\right) \leq C_{16}\left(\|P u\|_{T}^{(\tau)}+A_{1}(u)+T^{-1 / 2} R(u)\right) .
$$

From this inequality, (3.22), and (3.19) we see that when $\tau T^{2}>\tau_{3}$ and $T<T_{3}$, for $u \in \mathcal{S}_{T}\left(\boldsymbol{R}^{n}\right)$

$$
\begin{aligned}
& T^{-1 / 2} A_{1}\left(\Psi_{1} u\right) \leq \max \left(1, T^{1 / 2}\right)\left(C_{15}+C_{16}\right)\left(\|P u\|_{T}^{(r)}+A_{1}(u)+A_{1}\left(\Psi_{1} u\right)\right. \\
& \left.\quad+\left(T^{1 / 2}+T\right) A_{2}\left(\Psi_{1} u\right)+\left(T^{1 / 2}+T\right) T^{-1} R(u)\right) .
\end{aligned}
$$

Combining (3.13), (3.17), (3.23) we see that there exists a positive constant $C_{17}$ such that when $\tau T^{2}>\tau_{3}$ and $T<T_{3}$, for $u \in \mathcal{S}_{T}\left(\boldsymbol{R}^{n}\right)$

$$
\begin{aligned}
T^{-1 / 2} & A_{1}\left(\Psi_{1} u\right)+A_{2}\left(\Psi_{1} u\right)+A_{3}\left(\Psi_{1} u\right) \\
\leq & C_{17}\left(\|P u\|_{T}^{(T)}+A_{1}(u)+A_{1}\left(\Psi_{1} u\right)+T^{1 / 2} A_{2}\left(\Psi_{1} u\right)+T^{1 / 2} A_{3}(u)\right. \\
\quad & \left.+T^{-1} R(u)\right) .
\end{aligned}
$$

This completes the proof of (1). 
Proof of (2). Set

$$
\begin{gathered}
p_{0}(x, \xi)=p(x, \xi)-g\left(x, \xi^{\prime}\right), \\
P_{0}=p_{0}(x, D), \quad\left(P_{0}\right)_{(\beta)}^{(\alpha)}=\left(p_{0}\right)_{(\beta)}^{(\alpha)}(x, D) .
\end{gathered}
$$

Then we have that

$\left.A_{1}\left(\Psi_{1} u\right) \leq \sum_{1 \leq|\alpha|+|\beta| \leq 2}\left(\| E_{(|\alpha|-|\beta|) / 2}\left(P_{0}\right)_{(\beta)}^{(\alpha)}\right) \Psi_{1} u\left\|_{T}^{(\tau)}+\right\| E_{(|\alpha|-|\beta|) / 2} g_{(\beta)}^{(\alpha)}\left(x, D^{\prime}\right) \Psi_{1} u \|_{T}^{(\tau)}\right)$

We use the next two lemmas to estimate the right hand side of the above inequality. The assumption (III) is used to estimate the second terms in the parenthesis.

Lemma 3.11. Assume that (III) holds. Let $\chi \in S_{\phi, \varphi}^{0,0}$ with supp $\chi \subseteq \operatorname{supp} \psi_{0}$. Then

$$
\left\langle\xi^{\prime}\right\rangle^{(|\alpha|-|\beta|) / 2} \circ g_{(\beta)}^{(\alpha)} \circ \chi \in S_{1 / 2,1 / 2}^{3 / 2}\left(\mathbb{R}^{n} \times \mathbb{R}^{n-1}\right)
$$

for $\alpha \in \mathbb{Z}_{+}^{n}, \beta \in \mathbb{Z}_{+}^{n-1}$ with $|\alpha| \leq 2$.

Lemma 3.12. For $\alpha, \beta \in \mathbb{Z}_{+}^{n}$ with $|\alpha|+|\beta|=1$ or 2 we have that

$$
\left\langle\xi^{\prime}\right\rangle(|\alpha|-|\beta|) / 2 \circ\left(p_{0}\right)_{(\beta)}^{(\alpha)}=\sum_{k=1}^{2} a_{k} \circ L_{01} \circ L_{0 k}+\sum_{k=1}^{2} b_{k} \circ L_{0 k}+b_{0}
$$

with some $a_{k} \in S_{1,0}^{1 / 2}\left(\mathbb{R}^{n} \times \mathbb{R}^{n-1}\right), b_{k} \in S_{1,0}^{1}\left(\mathbb{R}^{n} \times \mathbb{R}^{n-1}\right)$ for $k \neq 0, b_{0} \in S_{1,0}^{3 / 2}\left(\mathbb{R}^{n} \times\right.$ $\left.\mathbb{R}^{n-1}\right)$.

From Lemma 3.12 there exists a positive constant $C_{1}$ such that for any $\tau, T$

$$
\begin{gathered}
\left\|E_{(|\alpha|-|\beta|) / 2}\left(P_{0}\right)_{(\beta)}^{(\alpha)} u\right\|_{T}^{(\tau)} \leq C_{1}\left(\sum_{k=1}^{2}\left\|E_{1 / 2}\left(L_{01} \circ L_{0 k}\right)(x, D) u\right\|_{T}^{(\tau)}+\sum_{k=1}^{2}\left\|E_{1} L_{0 k}(x, D) u\right\|_{T}^{(\tau)}\right. \\
\left.+\left\|E_{3 / 2} u\right\|_{T}^{(\tau)}\right), \quad u \in \mathcal{S}\left(\mathbb{R}^{n}\right) .
\end{gathered}
$$

Summing this for $\alpha, \beta$ with $|\alpha|+|\beta|=1,2$ we get that for any $\tau, T$, and $u \in S\left(\mathbb{R}^{n}\right)$

$$
\begin{aligned}
& T^{-1 / 2} \sum_{1 \leq|\alpha|+|\beta| \leq 2}\left\|E_{(|\alpha|-|\beta|) / 2}\left(P_{0}\right)_{(\beta)}^{(\alpha)} u\right\|_{T}^{(\tau)} \leq C_{2}\left(\sum_{k=1}^{2} T^{-1 / 2}\left\|E_{1 / 2}\left(L_{01} \circ L_{0 k}\right)(x, D) u\right\|_{T}^{(\tau)}\right. \\
& \left.\quad+T^{1 / 2} \sum_{k=1}^{2} T^{-1}\left\|E_{1} L_{0 k}(x, D) u\right\|_{T}^{(\tau)}+T T^{-3 / 2}\left\|E_{3 / 2} u\right\|_{T}^{(\tau)}\right) \\
& \leq C_{2} \max (1, T) B(u)
\end{aligned}
$$

Here $C_{2}=C_{1}\left(2 n+\left(\begin{array}{c}2 n+1 \\ 2\end{array}\right)\right)$. Thus from (3.24) and (3.25) we have that for any 
$\tau, T$, and $u \in \mathcal{S}\left(\boldsymbol{R}^{n}\right)$

$$
\begin{aligned}
& T^{-1 / 2} A_{1}\left(\Psi_{1} u\right) \leq C_{2} \max (1, T) B\left(\Psi_{1} u\right) \\
& \quad+T^{-1 / 2} \sum_{1 \leq|\alpha|+\mid \beta_{1} \leq 2}\left\|E_{(|\alpha|-|\beta|) / 2} g_{(\beta)}^{(\alpha)}\left(x, D^{\prime}\right) \Psi_{1} u\right\|_{T}^{(\tau)} .
\end{aligned}
$$

From Lemma 3.11 there exists a positive constant $C_{3}$ such that for any $\tau, T$, and $u \in \mathcal{S}\left(\boldsymbol{R}^{n}\right)$

$$
\sum_{|\alpha|+|\beta| \leq 2}\left\|E_{(|\alpha|-|\beta|) / 2} g_{(\beta)}^{(\alpha)}\left(x, D^{\prime}\right) \Psi_{1} u\right\|_{T}^{(\tau)} \leq C_{3}\left\|E_{3 / 2} u\right\|_{T}^{(\tau)} .
$$

From (3.26) and (3.27) we get that for any $\tau, T$, and $u \in \mathcal{S}\left(\boldsymbol{R}^{n}\right)$

$$
T^{-1 / 2} A_{1}\left(\Psi_{1} u\right) \leq C_{2} \max (1, T) B\left(\Psi_{1} u\right)+C_{3} T^{-1 / 2}\left\|E_{3 / 2} u\right\|_{T}^{(\tau)}
$$

Since $c\left(x, \xi^{\prime}\right)=\left(L_{01}-L_{02}\right)(x, \xi)$,

$$
\|\| c\left(x, D^{\prime}\right) u\left\|_{T, 2}^{(\tau)} \leq \sum_{k=1}^{2}\right\|\left\|L_{0 k}(x, D) u\right\|_{T, 2}^{(\tau)} .
$$

Thus

$$
\begin{aligned}
& B(u) \leq\|\| u\|\|_{T, 6}^{(\tau)}+\sum_{i \neq j} \tau^{-1}\left\|\left(L_{0 i} \circ L_{0 j}\right)(x, D) u\right\|\left\|_{T, 2}^{(\tau)}+\tau^{-1}\right\| L_{01}(x, D) u\|\|_{T, 2}^{(\tau)} \\
& \quad+2 \sum_{k=1}^{2} \tau^{-1 / 2}\|\| L_{0 k}(x, D) u\|\|_{T, 4}^{(\tau)} .
\end{aligned}
$$

Using Proposition 3.2 and Lemma 3.2 we see that there exist positive constants $\tau_{1}, T_{1}, C_{4}$ such that when $\tau T^{2}>\tau_{1}$ and $T<T_{1}$,

$$
B(u) \leq C_{4}\left\|P_{0} u\right\|_{T}^{(\tau)}, \quad u \in \mathcal{S}_{T}\left(\boldsymbol{R}^{n}\right) .
$$

From Lemma 3.3 there exists a positive constant $C_{5}$ such that for any $\tau, T$, and $u \in \mathcal{S}\left(\boldsymbol{R}^{n}\right)$

$$
\left\|P \Psi_{1} u\right\|_{T}^{(\tau)} \leq C_{5}\left(\|P u\|_{T}^{(\tau)}+A_{1}(u)+R(u)\right) .
$$

From (3.27), (3.29), and (3.30) we get that when $\tau T^{2}>\tau_{1}$ and $T<T_{1}$, for $u \in \mathcal{S}_{T}\left(\boldsymbol{R}^{n}\right)$

$$
\begin{aligned}
B\left(\Psi_{1} u\right) & \leq C_{4}\left(\left\|P \Psi_{1} u\right\|_{T}^{(\tau)}+\left\|g\left(x, D^{\prime}\right) \Psi_{1} u\right\|_{T}^{(\tau)}\right) \\
& \leq C_{4}\left\{C_{5}\left(\|P u\|_{T}^{(\tau)}+A_{1}(u)+R(u)\right)+C_{3}\left\|E_{3 / 2} u\right\|_{T}^{(\tau)}\right\} \\
& \leq C_{4}\left(C_{3}+C_{5}\right)\left(\|P u\|_{T}^{(\tau)}+A_{1}(u)+R(u)\right) .
\end{aligned}
$$

Combining (3.28) and (3.31) we get that when $\tau T^{2}>\tau_{1}$ and $T<T_{1}$, for $u \in_{T} \mathcal{S}\left(\boldsymbol{R}^{n}\right)$

$$
\begin{aligned}
T^{-1 / 2} A_{1}\left(\Psi_{1} u\right)+B\left(\Psi_{1} u\right) & \leq\left(C_{2} \max (1, T)+1\right) B\left(\Psi_{1} u\right)+C_{3} T^{-1 / 2}\left\|E_{3 / 2} u\right\|_{T}^{(\tau)} \\
& \leq C_{6}\left(\|P u\|_{T}^{(\tau)}+A_{1}(u)+R(u)\right)+C_{3} T^{-1 / 2} R(u)
\end{aligned}
$$


with $C_{6}=\left(C_{2} \max (1, T)+1\right) C_{4}\left(C_{3}+C_{5}\right)$. This completes the proof of (2).

\section{§4. Proof of Proposition $\mathbb{1} . \mathbb{1}$}

In this section we deduce Proposition 1.1 from Lemma 2.3 and Proposition 3.1. We define $p_{l}(x, \xi)$ from $P(x, \xi)$ by $(2.11)$ in the same manner as in the beginning of section 2 . We set

$$
\begin{gathered}
L_{01}^{(l)}(x, \xi)=\xi_{1}-\lambda_{l}\left(x, \xi^{\prime}\right), \\
L_{02}^{(l)}(x, \xi)=\xi_{1}-\lambda_{l}\left(x, \xi^{\prime}\right)-c_{l}\left(x, \xi^{\prime}\right)
\end{gathered}
$$

with the notations in (2.7) and (2.10). Then we define $A_{i}^{(l)}(u)$ for $i=1,2, l=$ $1,2, u \in \mathcal{S}\left(\boldsymbol{R}^{n}\right), \tau>1, T>0$ by

$$
\begin{gathered}
A_{1}^{(l)}(u)=\sum_{1 \leq|\alpha|+|\beta| \leq 2}\left\|E_{(|\alpha|-|\beta|) / 2}\left(p_{l}\right)_{(\beta)}^{(\alpha)}(x, D) u\right\|_{T}^{(\tau)}, \\
A_{2}^{(l)}(u)=T^{-1}\left(\sum_{k=1}^{2}\left\|E_{1} L_{0 k}^{(l)}(x, D) u\right\|_{T}^{(\tau)}+\left\|E_{1} c\left(x, D^{\prime}\right) u\right\|_{T}^{(\tau)}\right),
\end{gathered}
$$

and we use the notations $A_{1}(u)$ and $R(u)$ in Proposition 3.1.

We use a family of $C^{\infty}$-functions $\left\{\psi_{j k}\right\}_{k \in I}(j=0,1)$ on $\mathbb{R}^{n} \times \mathbb{R}^{n-1}$ in Lemma 2.3 and we set

$$
\Psi_{k}=\psi_{1 k}\left(x, D^{\prime}\right)
$$

Since for any $k \in I$ one can choose $l \in\{1,2\}$ so that one of the conditions (I), (II), (III) in $\S 3$ holds with $\Phi=\Phi_{l}, \varphi=\varphi_{l}, \psi_{i}=\psi_{i k}, p=p_{l}, \lambda=\lambda_{l}, c=c_{l}, g=g_{l}$, it follows from Proposition 3.1 that for any $k \in I$ there exist $l(k) \in\{1,2\}$ and positive constants $\tau^{(k)}, T^{(k)}, C^{(k)}$ such that the following condition holds: when $l=l(k)$ and $\tau T^{2}>\tau^{(k)}$ and $T<T^{(k)}$,

$$
\begin{aligned}
& T^{-1 / 2} A_{1}^{(l)}\left(\Psi_{k} u\right)+A_{2}^{(l)}\left(\Psi_{k} u\right)+\left\|\Psi_{k} u\right\|_{T, 6}^{(\tau)} \\
& \quad \leq C^{(k)}\left(\left\|p_{l}(x, D) u\right\|_{T}^{(\tau)}+A_{1}^{(l)}(u)+T^{1 / 2} A_{2}^{(l)}(u)+T^{-1} R(u)\right), u \in \mathcal{S}_{T}\left(\boldsymbol{R}^{n}\right) .
\end{aligned}
$$

Since $L_{01}^{(1)}(x, \xi)-L_{01}^{(2)}(x, \xi)=-\frac{2}{3} c\left(x, \xi^{\prime}\right)$ and $L_{02}^{(1)}(x, \xi)-L_{02}^{(2)}(x, \xi)=\frac{4}{3} c\left(x, \xi^{\prime}\right)$, we have for any $l, \tau, T$ that

$$
\sum_{m=1}^{2} \sum_{k=1}^{2}\left\|E_{1} L_{0 k}^{(m)}(x, D) u\right\|_{T}^{(T)} \leq 3 T A_{2}^{(l)}(u), \quad u \in \mathcal{S}\left(\mathbb{R}^{n}\right)
$$

This inequality and (4.1) imply that when $l=l(k)$ and $\tau>\tau^{(k)}$ and $T<T^{(k)}$,

$$
T^{-1} \sum_{m=1}^{2} \sum_{s=1}^{2}\left\|E_{1} L_{0 s}^{(m)}(x, D) \Psi_{k} u\right\|_{T}^{(\tau)}
$$




$$
\begin{aligned}
& \leq 2 C^{(k)}\left(\left\|p_{l}(x, D) u\right\|_{T}^{(\tau)}+A_{1}^{(l)}(u)+T^{1 / 2} A_{2}^{(l)}(u)+T^{-1} R(u)\right) \\
& \text { for } u \in \mathcal{S}_{T}\left(\mathbb{R}^{n}\right) .
\end{aligned}
$$

To estimate $A_{1}(u)$ we need

Lemma 4.1. There exists a positive constant $C$ such that for any $l, \tau, T$

$$
\sum_{1 \leq|\alpha|+|\beta| \leq 2}\left\|E_{(|\alpha|-|\beta|) / 2}\left(P_{(\beta)}^{(\alpha)}-\left(p_{l}\right)_{(\beta)}^{(\alpha)}\right)(x, D) u\right\|_{T}^{(\tau)} \leq C R(u), \quad u \in \mathcal{S}\left(\boldsymbol{R}^{n}\right) .
$$

Proof. Using the equality (2.12) it can be easily checked that when $|\alpha|+$ $|\beta|=1$ or 2 ,

$$
\left\langle\xi^{\prime}\right\rangle^{(|\alpha|-|\beta|) / 2}\left(P_{(\beta)}^{(\alpha)}-\left(p_{l}\right)_{(\beta)}^{(\alpha)}\right)(x, \xi)=\sum_{k=0}^{2-\alpha_{1}} a_{k}\left(x, \xi^{\prime}\right) \xi_{1}{ }^{k}
$$

with some $a_{k} \in S_{1,0}^{(3 / 2)-k}\left(\boldsymbol{R}^{n} \times \boldsymbol{R}^{n-1}\right)$. This implies the lemma.

From Lemma 4.1 there exists a positive constant $M_{1}$ such that (4.3) with $C=M_{1}$ holds for any $l, \tau, T$. From Lemma 3.4 there exists a positive constant $M_{2}$ such that we have for any $k \in I$ that

$$
R\left(\Psi_{k} u\right) \leq M_{2} R(u), \quad u \in \mathcal{S}\left(\mathbb{R}^{i t}\right)
$$

Then we have for any $k \in I$ that when $l=l(k)$ and $\tau T^{2}>\tau^{(k)}$ and $T<T^{(k)}$,

$$
\begin{aligned}
& T^{-1 / 2} A_{1}\left(\Psi_{k} u\right) \leq T^{-1 / 2} A_{1}^{(l)}\left(\Psi_{k} u\right)+T^{-1 / 2} M_{1} R\left(\Psi_{k} u\right) \\
& \leq C^{(k)}\left(\left\|p_{l}(x, D) u\right\|_{T}^{(\tau)}+A_{1}^{(l)}(u)+T^{1 / 2} A_{2}^{(l)}(u)\right) \\
&+\left(C^{(k)}+M_{1} M_{2} T^{1 / 2}\right) T^{-1} R(u) \\
& \text { for } u \in \mathcal{S}_{T}\left(\mathbb{R}^{n}\right) .
\end{aligned}
$$

To estimate $\left\|\left(p_{l}-P\right)(x, D) u\right\|_{T}^{(\tau)}$ we need

Lemma 4.2. There exist positive constants $\tau_{0}, T_{0}, C_{0}$ such that when $\tau T^{2}>\tau_{0}$ and $T<T_{0}$, for any $l$ and $u \in \mathcal{S}_{T}\left(\mathbb{R}^{n}\right)$

$$
\begin{aligned}
\left\|\left(p_{l}-P\right)(x, D) u\right\|_{T}^{(\tau)} \leq C_{0}\left(\tau^{-1 / 2}\|P(x, D) u\|_{T}^{(\tau)}\right. & +R(u)+T A_{2}^{(l)}(u) \\
& \left.+T^{2}\|u\|_{T, 4}^{(\tau)}\right) .
\end{aligned}
$$

Proof. We recall (2.12). It is easy to see that

$$
\begin{aligned}
& {\left[\sum_{j=1}^{2} b_{l j}\left(x, \xi^{\prime}\right)\left(\xi_{1}-\lambda_{l}\left(x, \xi^{\prime}\right)\right)^{j}\right](x, D)} \\
& \quad=\sum_{j=1}^{2} b_{l j}(x, D) L_{01}^{(l)}(x, D)^{j}+r_{01}\left(x, D^{\prime}\right) D_{1}+r_{l 2}\left(x, D^{\prime}\right)
\end{aligned}
$$

with some $r_{l 1} \in S_{1,0}^{0}\left(\boldsymbol{R}^{n} \times \boldsymbol{R}^{n-1}\right), r_{l 2} \in S_{1,0}^{1}\left(\mathbb{R}^{n} \times \mathbb{R}^{n-1}\right)$. 
Thus there exists a positive constant $C_{1}$ such that for any $\tau, T$, and $u \in \mathcal{S}\left(\mathbb{R}^{n}\right)$

$$
\left\|\left(p_{l}-P\right)(x, D) u\right\|_{T}^{(\tau)} \leq C_{1}\left(\sum_{j=1}^{2}\left\|E_{2-j} L_{01}^{(l)}(x, D)^{j} u\right\|_{T}^{(\tau)}+\left\|D_{1} u\right\|_{T}^{(\tau)}+\left\|E_{1} u\right\|_{T}^{(\tau)}\right) .
$$

Using Parseval's formula we can easily see that

$$
\left\|E_{s} u\right\|_{T}^{(\tau)} \leq\left\|E_{s^{\prime}} u\right\|_{T}^{(\tau)} \quad \text { if } \quad s^{\prime} \geq s .
$$

So from (4.6) we have for any $\tau, T$, and $u \in \mathcal{S}\left(\mathbb{R}^{n}\right)$ that

$$
\begin{aligned}
\left\|\left(p_{l}-P\right)(x, D) u\right\|_{T}^{(\tau)} & \leq C_{1}\left(\left\|L_{01}^{(l)}(x, D)^{2} u\right\|_{T}^{(\tau)}+\right. \\
& \left.+\left\|E_{1} L_{01}^{(l)}(x, D) u\right\|_{T}^{(\tau)}+\left\|E_{3 / 2} u\right\|_{T}^{(\tau)}\right) \\
\leq & C_{1}\left(\left\|L_{01}^{(l)}(x, D)^{2} u\right\|_{T}^{(\tau)}+T A_{2}^{(l)}(u)+R(u)\right) .
\end{aligned}
$$

We have that

$$
\begin{aligned}
L_{01}^{(2)}(x, D)^{2}=L_{01}^{(1)}(x, D)^{2} & +\frac{4}{3} c\left(x, D^{\prime}\right) L_{01}^{(1)}(x, D)+\frac{2}{3}\left(D_{x_{1}} c\right)\left(x, D^{\prime}\right) \\
& -\frac{2}{3}\left[\lambda\left(x, D^{\prime}\right), c\left(x, D^{\prime}\right)\right]+\frac{4}{9} c\left(x, D^{\prime}\right)^{2} .
\end{aligned}
$$

Thus there exists a positive constant $C_{2}$ such that for any $\tau, T$, and $u \in \mathcal{S}\left(\mathbb{R}^{n}\right)$

$$
\begin{gathered}
\left\|L_{01}^{(2)}(x, D)^{2} u\right\|_{T}^{(\tau)} \leq \\
\quad\left\|L_{01}^{(1)}(x, D)^{2} u\right\|_{T}^{(\tau)}+C_{2}\left(\left\|E_{1} L_{01}^{(1)}(x, D) u\right\|_{T}^{(\tau)}\right. \\
\left.+\left\|E_{1} c\left(x, D^{\prime}\right) u\right\|_{T}^{(\tau)}+\left\|E_{1} u\right\|_{T}^{(\tau)}\right) .
\end{gathered}
$$

Using (4.7) we see from this inequality that for any $\tau, T$, and $u \in S\left(\mathbb{R}^{n}\right)$

$$
\left\|L_{01}^{(2)}(x, D)^{2} u\right\|_{T}^{(\tau)} \leq\left\|L_{01}^{(1)}(x, D)^{2} u\right\|_{T}^{(T)}+C_{2}\left(T A_{2}^{(l)}(u)+R(u)\right) .
$$

Combining this inequality and (4.8) we obtain that for any $\tau, T$, and $u \in \mathcal{S}\left(\mathbb{R}^{n}\right)$

$$
\left\|\left(p_{l}-P\right)(x, D) u\right\|_{T}^{(\tau)} \leq C_{1}\left\|L_{01}^{(1)}(x, D)^{2} u\right\|_{T}^{(\tau)}+C_{1}\left(C_{2}+1\right)\left(T A_{2}^{(l)}(u)+R(u)\right) .
$$

Note that $p(x, \xi)=L_{01}^{(1)}(x, \xi)^{2} L_{01}^{(2)}(x, \xi)$. Thus using Proposition 3.1 and Lemma 3.2 we see that there exist positive constants $\tau_{1}, T_{1}, C_{3}$ such that when $\tau T^{2}>\tau_{1}$ and $T<T_{1}$,

$$
\left\|L_{01}^{(1)}(x, D)^{2} u\right\|_{T}^{(\tau)} \leq C_{3} \tau^{-1 / 2}\|p(x, D) u\|_{T}^{(\tau)}, \quad u \in \mathcal{S}_{T}\left(\mathbb{R}^{n}\right) .
$$

There exists a positive constant $C_{4}$ such that for any $\tau, T$, and $u \in \mathcal{S}\left(\mathbb{R}^{n}\right)$

$$
\|q(x, D) u\|_{T}^{(\tau)} \leq C_{4} \sum_{k=0}^{2}\left\|E_{2-k} D_{1}^{k} u\right\|_{T}^{(\tau)} .
$$

Since $P=p+q$, combining this inequality and (4.10) we obtain that when 
$\tau T^{2}>\tau_{1}$ and $T<T_{1}$

$\left\|L_{01}^{(1)}(x, D)^{2} u\right\|_{T}^{(\tau)} \leq C_{3} \tau^{-1 / 2}\|P(x, D) u\|_{T}^{(\tau)}+C_{3} C_{4} T^{2}\|u\| \|_{T, 4}^{(\tau)}, u \in \mathcal{S}_{T}\left(\boldsymbol{R}^{n}\right)$.

(4.9) and (4.11) imply the lemma.

From Lemma 4.2 there exist positive constants $\tau_{1}>\max _{k \in I} \tau^{(k)}, T_{1}<\min _{k \in I}$ $T^{(k)}$, and $M_{3}$ such that when $\tau T^{2}>\tau_{1}$ and $T<T_{1}$, for any $l$ and $u \in \mathcal{S}_{T}\left(\boldsymbol{R}^{n}\right)$

$$
\left\|p_{l}(x, D) u\right\|_{T}^{(\tau)} \leq M_{3}\left(\|P(x, D) u\|_{T}^{(\tau)}+R(u)+T A_{2}^{(l)}(u)+T^{2}\|u\|_{T, 4}^{(\tau)}\right) .
$$

Combining (4.1), (4.2), (4.5), and (4.12) we see that there exists a positive constant $M_{4}$ such that when $\tau T^{2}>\tau_{1}$ and $T<T_{1}$, for any $l$ and $u \in \mathcal{S}_{T}\left(\boldsymbol{R}^{n}\right)$

$$
\begin{aligned}
T^{-1 / 2} & A_{1}\left(\Psi_{k} u\right)+\sum_{m=1}^{2} A_{2}^{(m)}\left(\Psi_{k} u\right)+\left\|\mid \Psi_{k} u\right\|_{T, 6}^{(\tau)} \\
\leq & M_{4}\left(\|P(x, D) u\|_{T}^{(\tau)}+A_{1}^{(l(k))}(u)+T^{1 / 2} A_{2}^{(l(k))}(u)+T^{-1} R(u)\right. \\
& \left.+T^{2}\|u\| \|_{T, 4}^{(\tau)}\right) .
\end{aligned}
$$

Since $\sum_{k \in I} \Psi_{1 k} u=u$ for $u \in \mathcal{S}\left(\boldsymbol{R}^{n}\right)$, we have that

$$
\begin{aligned}
& T^{-1 / 2} A_{1}(u)+\sum_{m=1}^{2} A_{2}^{(m)}(u)+\|\| u\|\|_{T, 6}^{(\tau)} \\
& \quad \leq \sum_{k \in I}\left(T^{-1 / 2} A_{1}\left(\Psi_{k} u\right)+\sum_{m=1}^{2} A_{2}^{(m)}\left(\Psi_{k} u\right)+\left\|\Psi_{k} u\right\|_{T, 6}^{(\tau)}\right), \quad u \in \mathcal{S}\left(\boldsymbol{R}^{n}\right) .
\end{aligned}
$$

(4.13) and this inequality imply that when $\tau T^{2}>\tau_{1}$ and $T<T_{1}$, for $u \in \mathcal{S}_{T}\left(\boldsymbol{R}^{n}\right)$

$$
\begin{aligned}
T^{-1 / 2} & A_{1}(u)+\sum_{m=1}^{2} A_{2}^{(m)}(u)+\|\| u \|_{T, 6}^{(\tau)} \\
\leq & M_{4}\left(\#(I)\|P(x, D) u\|_{T}^{(\tau)}+\sum_{k \in I} A_{1}^{(l(k))}(u)+T^{1 / 2} \sum_{k \in I} A_{2}^{(l(k))}(u)+\#(I) T^{-1} R(u)\right. \\
& \left.\quad+\#(I) T^{2}\|u\| \|_{T, 4}^{(\tau)}\right) .
\end{aligned}
$$

Since $A_{1}^{(l)}(u) \leq A_{1}(u)+M_{1} R(u)$ for any $l$ and $u \in \mathcal{S}\left(\boldsymbol{R}^{n}\right)$, the above inequality implies that when $\tau T^{2}>\tau_{1}$ and $T<T_{1}$, for $u \in \mathcal{S}_{T}\left(\boldsymbol{R}^{n}\right)$

$$
\begin{aligned}
T^{-1 / 2} & A_{1}(u)+\sum_{m=1}^{2} A_{2}^{(m)}(u)+\|\| u \|_{T, 6}^{(\tau)} \\
\leq & M_{4} \#(I)\left\{\|P(x, D) u\|_{T}^{(\tau)}+A_{1}(u)+T^{1 / 2} \sum_{m=1}^{2} A_{2}^{(m)}(u)+\left(M_{1} T+1\right) T^{-1} R(u)\right. \\
& \left.\quad+T^{2}\|\| u\|\|_{T, 4}^{(\tau)}\right\} .
\end{aligned}
$$

To complete the proof of Proposition 1.1 we need 
Lemma 4.3. There exists a positive constant $C$ such that we have for any $\tau, T$ that

$$
R(u) \leq \frac{2}{\tau T}\|P(x, D) u\|_{T}^{(\tau)}+C T^{3 / 2}\|\| u \|_{T, 5}^{(\tau)}
$$

for $u \in \mathcal{S}_{T / 2}\left(\mathbb{R}^{n}\right)$.

Proof. An integration by parts gives that for any $\tau, T$

$$
\left\|D_{1} u\right\|_{T}^{(\tau)} \geq \frac{\tau T}{2}\|u\|_{T}^{(\tau)}, \quad u \in \mathcal{S}_{T / 2}\left(\mathbb{R}^{n}\right)
$$

Substituting $E_{-1 / 2} D_{1}^{2} u$ into $u$ yields that

$$
\left\|D_{1}^{3} E_{-1 / 2} u\right\|_{T}^{(\tau)} \geq \frac{\tau T}{2}\left\|E_{-1 / 2} D_{1}^{2} u\right\|_{T}^{(\tau)}, \quad u \in \mathcal{S}_{T / 2}\left(\mathbb{R}^{n}\right) .
$$

We have that $P(x, D)=D_{1}^{3}+\sum_{i=0}^{2} a_{i}\left(x, D^{\prime}\right) D_{1}^{i}$ with some $a_{i} \in S_{1,0}^{3-i}\left(\mathbb{R}^{n} \times \mathbb{R}^{n-1}\right)$, then

$$
\begin{aligned}
& D_{1}^{3} E_{-1 / 2} u=E_{-1 / 2} D_{1}^{3} u \\
& \quad=E_{-1 / 2} P(x, D) u-\sum_{i=0}^{2} E_{-1 / 2} a_{i}\left(x, D^{\prime}\right) D_{1}^{i} u \text { for } u \in \mathcal{S}\left(\mathbb{R}^{n}\right)
\end{aligned}
$$

Using the fact that $\left\langle\xi^{\prime}\right\rangle^{-1 / 2} \circ a_{i} \in S_{1,0}^{(5 / 2)-i}\left(\mathbb{R}^{n} \times \mathbb{R}^{n-1}\right)$ and applying (4.7) to the first term in (4.16) we see that there exists a positive constant $C$ such that for any $\tau, T$, and $u \in \mathcal{S}\left(\mathbb{R}^{n}\right)$

$$
\left\|D_{1}^{3} E_{-1 / 2} u\right\|_{T}^{(\tau)} \leq\left\|P^{P}(x, D)\right\|_{T}^{(\tau)}+C \sum_{i=0}^{2}\left\|E_{(5 / 2)-i} D_{1}^{i} u\right\|_{T}^{(\tau)}
$$

(4.15) and (4.16) imply that for any $\tau, T$, and $u \in \mathcal{S}_{T / 2}\left(\mathbb{R}^{n}\right)$

$$
\left\|E_{-1 / 2} D_{1}^{2} u\right\|_{T}^{(\tau)} \leq \frac{2}{\tau T}\|P(x, D) u\|_{T}^{(\tau)}+2 C T^{3 / 2}\|\| u \|_{T, 5}^{(\tau)} .
$$

Therefore, for any $\tau, T$, and $u \in \mathcal{S}_{T / 2}\left(\mathbb{R}^{n}\right)$

$$
\begin{aligned}
R(u) & =\left\|E_{-1 / 2} D_{1}^{2} u\right\|_{T}^{(\tau)}+\sum_{i=1}^{2}\left\|E_{-1 / 2+i} D_{1}^{2-i} u\right\|_{T}^{(\tau)} \\
& \leq\left(\frac{2}{\tau T}\|P(x, D) u\|_{T}^{(\tau)}+2 C T^{3 / 2}\|u\| \|_{T, 5}^{(\tau)}\right)+T^{3 / 2}\|\| u \|_{T, 3}^{(\tau)} \\
& \leq \frac{2}{\tau T}\|P(x, D) u\|_{T}^{(\tau)}+(2 C+1) T^{3 / 2}\|\| u \|_{T, 5}^{(\tau)} .
\end{aligned}
$$

This completes the proof.

We take a positive number $M_{2}$ such that the inequality in Lemma 4.3 
holds with $C=M_{2}$. Then (4.14) implies that when $\tau T^{2}>\tau_{1}$ and $T<T_{1}$, for $u \in \mathcal{S}_{T / 2}\left(\boldsymbol{R}^{n}\right)$

$$
\begin{aligned}
T^{-1 / 2} & A_{1}(u)+\sum_{m=1}^{2} A_{2}^{(m)}(u)+\|u\|_{T, 6}^{(\tau)} \\
\leq & M_{4} \#(I)\left[\left\{1+\left(M_{1} T+1\right) \frac{1}{\tau T^{2}}\right\}\|P(x, D) u\|_{T}^{(\tau)}+A_{1}(u)+T^{1 / 2} \sum_{m=1}^{2} A_{2}^{(m)}(u)\right. \\
& \left.+\left(M_{1} T+1\right) T^{1 / 2}\|\| u\left\|\left.\right|_{T, 5} ^{(T)}+T^{2}\right\|\|u\|_{T, 4}^{(\tau)}\right] \\
\leq & M_{4} \#(I)\left(1+\frac{M_{1} T_{1}+1}{\tau_{1}}\right)\|P(x, D)\|_{T}^{(\tau)}+M_{4}(I)\left\{A_{1}(u)+T^{1 / 2} \sum_{m=1}^{2} A_{2}^{(m)}(u)\right. \\
& \left.+\left(M_{1} T_{1}+1\right) T^{1 / 2}\|u\|\left\|_{T, 4}^{(\tau)}+T^{2}\right\| u \|_{T, 5}^{(\tau)}\right\} .
\end{aligned}
$$

The second term on the right hand side can be absorbed into the left hand side by decreasing $T$. Therefore, we have proved Proposition 1.1.

\section{§. Pseudodifferential Operators}

In this section we collect the facts on the pseudodifferential operators which we use in this paper. In this paper we use the classes of symbols $S_{\Phi, \varphi}^{M, m}$ with $(\Phi, \varphi)$ stated after Lemma 2.2 which contain $S_{\rho, 1-\rho}^{d}\left(\boldsymbol{R}^{n} \times \boldsymbol{R}^{n-1}\right), \frac{1}{2} \leq \rho \leq 1$.

Definition 5.1. Let $(\Phi, \varphi)$ be a pair of weight functions satisfying (2.31) (2.23). And let $a \in S_{\Phi, \varphi}^{M, m}$. We define an operator $a\left(x, D^{\prime}\right)$ on $\mathcal{S}\left(\boldsymbol{R}^{n}\right)$ by the standard formula

$$
a\left(x, D^{\prime}\right) u=(2 \pi)^{-(n-1)} \int e^{i x^{\prime} \cdot \xi^{\prime}} a\left(x, \xi^{\prime}\right) \hat{u}\left(x_{1}, \xi^{\prime}\right) d \xi^{\prime}
$$

where $\hat{u}\left(x_{1}, \xi^{\prime}\right)$ denotes the partial Fourier transform of $u$ in $x^{\prime}$.

$a\left(x, D^{\prime}\right)$ transforms $\mathcal{S}\left(\boldsymbol{R}^{n}\right)$ into $\mathcal{S}\left(\boldsymbol{R}^{n}\right)$ and $\mathcal{S}_{T}\left(\boldsymbol{R}^{n}\right)$ into $\mathcal{S}_{T}\left(\boldsymbol{R}^{n}\right)$. If $(\Phi, \varphi)$ is a pair of weight functions, pairs of functions on $\boldsymbol{R}^{n-1} \times \boldsymbol{R}^{n-1},\left(\Phi\left(x_{1}, \cdot\right)\right.$, $\left.\varphi\left(x_{1}, \cdot\right)\right)\left(x_{1} \in \boldsymbol{R}\right)$ satisfy uniformly the conditions for weight functions of BealsFeefferman's class. This follows from $(2.31) \sim(2.33)$ and the following lemma.

Lemma 5.1. There exist positive constants $M, \delta$ satisfying the following condition.

$$
M^{-1} \leq \Phi_{t}\left(x^{\prime}, \xi^{\prime}\right) / \Phi_{t}\left(y^{\prime}, \eta^{\prime}\right) \leq M, \quad M^{-1} \leq \varphi_{t}\left(x^{\prime}, \xi^{\prime}\right) / \varphi_{t}\left(y^{\prime}, \eta^{\prime}\right) \leq M
$$

for any $\left(y^{\prime}, \eta^{\prime}\right) \in U_{t}\left(x^{\prime}, \xi^{\prime}\right)$ and $t \in \mathbb{R}$ where

$$
\begin{aligned}
& U_{t}\left(x, \xi^{\prime}\right)=\left\{\left(y^{\prime}, \eta^{\prime}\right) \in \boldsymbol{R}^{n-1} \times \boldsymbol{R}^{n-1} ;\left|x^{\prime}-y^{\prime}\right|<\delta \varphi_{t}\left(\left(x^{\prime}, \xi^{\prime}\right),\left|\xi^{\prime}-\eta^{\prime}\right|<\delta \Phi_{t}\left(x^{\prime}, \xi^{\prime}\right)\right\}\right. \\
& \Phi_{t}\left(x^{\prime}, \xi^{\prime}\right)=\Phi\left(\left(t, x^{\prime}\right), \xi^{\prime}\right), \varphi_{t}\left(x^{\prime}, \xi^{\prime}\right)=\varphi\left(\left(t, x^{\prime}\right), \xi^{\prime}\right) .
\end{aligned}
$$


Proof. Using Taylor's formula and (2.31), (2.32) we see that there exists a positive constant $C_{0}$ such that when $C \delta<\frac{1}{2}$ with the constant $C$ in (2.31).

$$
\begin{aligned}
& \left|\Phi_{t}\left(y^{\prime}, \eta^{\prime}\right)-\Phi_{t}\left(x^{\prime}, \xi^{\prime}\right)\right| \leq C_{0} \delta \Phi_{t}\left(x^{\prime}, \xi^{\prime}\right), \\
& \left|\varphi_{t}\left(y^{\prime}, \eta^{\prime}\right)-\varphi_{t}\left(x^{\prime}, \xi^{\prime}\right)\right| \leq C_{0} \delta \varphi_{t}\left(x^{\prime}, \xi^{\prime}\right)
\end{aligned}
$$

for any $\left(y^{\prime}, \eta^{\prime}\right) \in U_{t}\left(x^{\prime}, \xi^{\prime}\right)$. This implies the lemma.

Remark 5.1. We define semi-norms $|\cdot|_{N}^{M, m}$ in $S_{\Phi, \varphi}^{M, m}$ for $N \in \mathbb{Z}_{+}$by

$$
a \mapsto|a|_{N}^{M, m}=\max _{|\alpha|+|\beta| \leq N} \sup _{\left(x, \xi^{\prime}\right) \in \boldsymbol{R}^{n} \times \boldsymbol{R}^{n-1}}\left[\left|a_{(\alpha)}^{(\beta)}\left(x, \xi^{\prime}\right)\right|\left(\Phi^{-M+|\beta|} \varphi^{-m+|\alpha|}\right)\left(x, \xi^{\prime}\right)\right] .
$$

Then $S_{\Phi, \varphi}^{M, m}$ becomes a Frechet space by the topology defined by these semi-norms.

Lemma 5.2. Let $a \in S_{\varphi, \varphi}^{M, m}$ and $b \in S_{\phi, \varphi}^{M^{\prime}, m^{\prime \prime}}$. Then if we define $a \circ b \in C^{\infty}\left(\mathbb{R}^{n} \times\right.$ $\left.\mathbb{R}^{n-1}\right)$ by the formula

$$
(a \circ b)\left(x, \xi^{\prime}\right)=(2 \pi)^{-(n-1)} O S-\iint e^{-i\left(y^{\prime}-x^{\prime}\right) \cdot\left(\eta^{\prime}-\xi^{\prime}\right)} a\left(x, \eta^{\prime}\right) b\left(\left(x_{1}, y^{\prime}\right), \xi^{\prime}\right) d y^{\prime} d \eta^{\prime},
$$

we have that $a \circ b \in S_{\Phi, \varphi}^{M+M^{\prime}, m+m^{\prime}}$ and $(a \circ b)\left(x, D^{\prime}\right)=a\left(x, D^{\prime}\right) b\left(x, D^{\prime}\right)$. Moreover, we have an asymptotic expansion that for any $N \in \mathbb{N}$

$$
(a \circ b)\left(x, \xi^{\prime}\right)=\sum_{|\alpha|<N} \frac{1}{\alpha !}\left(\partial_{\xi^{\prime}}^{\alpha} a D_{x^{\prime}}^{a} b\right)\left(x, \xi^{\prime}\right)+r_{N}[a, b]\left(x, \xi^{\prime}\right)
$$

with

$$
\begin{gathered}
r_{N}[a, b]\left(x, \xi^{\prime}\right)=\int_{0}^{1} r_{N \theta}[a, b]\left(x, \xi^{\prime}\right)(1-\theta)^{N-1} d \theta, \\
r_{N \theta}[a, b]\left(x, \xi^{\prime}\right)=N \sum_{|\alpha|=N} \frac{1}{\alpha !}(2 \pi)^{-(\xi z-1)} \iint e^{-i\left(y^{\prime}-x^{\prime}\right) \cdot\left(\eta^{\prime}-\xi^{\prime}\right)}\left(\partial_{\xi^{\prime}}^{\alpha} a\right)\left(x, \xi^{\prime}\right. \\
\left.+\theta\left(\eta^{\prime}-\xi^{\prime}\right)\right) \times D_{y^{\prime}}^{\alpha} b\left(\left(x_{1}, y^{\prime}\right), \xi^{\prime}\right) d y^{\prime} d \eta^{\prime},
\end{gathered}
$$

$\left\{r_{N \theta}[a, b]\right\}_{\theta \in[0,1]}$ is a bounded set in $S_{\Phi, \varphi}^{M+M^{\prime}-N, m+m^{\prime}-N}$.

Proof. When $a, b \in C_{0}^{\infty}\left(\mathbb{R}^{n} \times \mathbb{R}^{n-1}\right)$, it is not hard to check (5.2) with the notations (5.1), (5.3), (5.4). Now let us consider an oscillatory integral $h_{\theta}[a, b]\left(x, \xi^{\prime}\right)=(2 \pi)^{-(n-1)} O S-\iint e^{-i y^{\prime} \cdot \eta^{\prime}} a\left(x, \xi^{\prime}+\theta \eta^{\prime}\right) b\left(\left(x_{1}, y^{\prime}+x^{\prime}\right), \xi^{\prime}\right) d y^{\prime} d \eta^{\prime}$ where $a \in S_{\phi, \varphi}^{M, m}, b \in S_{\phi, \varphi}^{M \mu^{\prime} m^{\prime}}, \theta \in[0,1]$.

Claim. (1) $h_{\theta}[a, b] \in S_{\Phi, \varphi}^{M+M^{\prime}, m+m^{\prime}}$ for all $\theta$ and for any $L \in \mathbb{N}$ there exist positive constant $C$ and $P \in \mathbb{N}$ depending only on $L, M, m, M^{\prime}, m^{\prime}, \Phi, \varphi$ such that 


$$
\sup _{\theta \in[0,1]}\left|\partial_{x}^{\alpha} \partial_{\xi^{\prime}}^{\beta} h_{\theta}[a, b]\left(x, \xi^{\prime}\right)\right| \leq|a|_{P}^{M, m}|b|_{P}^{M^{\prime}, m^{\prime}}\left(\Phi^{M+M^{\prime}-|\beta|} \varphi^{m+m^{\prime}-|\alpha|}\right)\left(x, \xi^{\prime}\right)
$$

for any $\alpha, \beta$ with $|\alpha|+|\beta| \leq L$.

(2) Let $\left\{a_{k}\right\}_{k=1}^{\infty}$ and $\left\{b_{k}\right\}_{k=1}^{\infty}$ be bounded sets respectively in $S_{\varphi, \varphi}^{M, m}$ and $S_{\phi, \varphi}^{M \prime, m^{\prime}}$ such that there exist $a \in S_{\emptyset, \varphi}^{M, m}$ and $b \in S_{\phi, \varphi}^{M \prime, m \prime}$ such that $a_{k} \rightarrow a(k \rightarrow \infty)$ and $b_{k} \rightarrow$ $b(k \rightarrow \infty)$ in $C^{\infty}\left(\boldsymbol{R}^{n} \times \boldsymbol{R}^{n-1}\right)$. Then $h_{\theta}\left[a_{k}, b_{k}\right]\left(x, \xi^{\prime}\right) \rightarrow h_{\theta}[a, b]\left(x, \xi^{\prime}\right)(k \rightarrow \infty)$ for any $\theta$ and $\left(x, \xi^{\prime}\right)$, and $\left\{h_{\theta}\left[a_{k}, b_{k}\right]\right\}_{k \in N, \theta \in[0,1]}$ is bounded in $S_{\varphi, \varphi}^{M+M^{\prime}, m+m^{\prime}}$.

Proof of claim. Set $f_{\theta}[a, b]\left(x, y^{\prime}, \xi^{\prime}, \eta^{\prime}\right)=a\left(x, \xi^{\prime}+\theta \eta^{\prime}\right) b\left(\left(x_{1}, y^{\prime}+x^{\prime}\right), \xi^{\prime}\right)$. Then

$$
\begin{aligned}
\mid \partial_{y^{\prime}}^{\alpha} \partial_{\eta^{\prime}}^{\beta}, & \left.f_{\theta}[a, b]\left(x, y^{\prime}, \xi^{\prime}, \eta^{\prime}\right)|\leq| a\right|_{|\beta|} ^{M, m}|b|_{|\alpha|}^{\left.M^{\prime}\right|^{m^{\prime}}}\left(\Phi^{M-|\beta|} \varphi^{m}\right)\left(x, \xi^{\prime}+\theta \eta^{\prime}\right) \\
& \times\left(\Phi^{M^{\prime}} \varphi^{m^{\prime}-|\alpha|}\right)\left(\left(x_{1}, y^{\prime}+x^{\prime}\right), \xi^{\prime}\right) \\
\leq & C_{1}|a|_{|\beta|}^{M_{0} m}|b|_{|\alpha|}^{M^{\prime}, m^{\prime}}\left\langle\xi^{\prime}\right\rangle|M|+(|m|) / 2+\left|M^{\prime}\right|+\left(\left|m^{\prime}\right|\right) / 2+(|\alpha|) / 2 \\
& \times\left\langle\eta^{\prime}\right\rangle^{|M|+(|m|) / 2}
\end{aligned}
$$

with $C_{1}$ depending only on $M, m, M^{\prime}, m^{\prime}, \alpha, \beta, \Phi, \varphi$. From this inequality Leibniz rule shows that if $L, N \in N$, one can find $C_{2}$ depending only on $L, N$, $M, m, M^{\prime}, m^{\prime}, \Phi, \varphi$ such that

$$
\begin{aligned}
& \left|\left\langle y^{\prime}\right\rangle^{-2 L}\left(1-\Delta_{\eta^{\prime}}\right)^{L}\left[\left\langle\eta^{\prime}\right\rangle^{-2 N}\left(1-\Delta_{y^{\prime}}\right)^{N} f_{\theta}[a, b]\left(x, y^{\prime}, \xi^{\prime}, \eta^{\prime}\right)\right]\right| \\
& \leq C_{2}|a|_{2(N+L)}^{M, m}|b|_{2(N+L)}^{M m^{\prime}, m^{\prime}}\left\langle\xi^{\prime}\right\rangle|M|+(|m|) / 2+\left|M^{\prime}\right|+\left(\left|m^{\prime}\right|\right) / 2+N+L \\
& \times\left\langle\eta^{\prime}\right\rangle^{-2 N+|M|+(|m|) / 2}\left\langle y^{\prime}\right\rangle^{-2 L} .
\end{aligned}
$$

It also follows from the estimate (5.6) that if $L, N \in N$ satisfy that

$$
-2 N+|M|+\frac{|m|}{2}<-(n-1), \quad-2 L<-(n-1)
$$

we have that

$$
\begin{aligned}
& h_{\theta}[a, b]\left(x, \xi^{\prime}\right)=(2 \pi)^{-(n-1)} \iint e^{-i y^{\prime} \cdot \eta^{\prime}}\left\langle y^{\prime}\right\rangle^{-2 L} \\
& \quad \times\left(1-\Delta_{\eta^{\prime}}\right)^{L}\left[\left\langle\eta^{\prime}\right\rangle^{-2 N}\left(1-\Delta_{y^{\prime}}\right)^{N} f_{\theta}[a, b]\left(x, y^{\prime}, \xi^{\prime}, \eta^{\prime}\right)\right] d y^{\prime} d \eta^{\prime} .
\end{aligned}
$$

We shall show (2). From the estimates (5.7) with $a=a_{k}, b=b_{k}$, and $L, N$ satisfying (5.8), and from the fact that $f_{\theta}\left[a_{k}, b_{k}\right]\left(x, \cdot, \xi^{\prime}, \cdot\right) \rightarrow f_{\theta}[a, b]\left(x, \cdot, \xi^{\prime}, \cdot\right)$ in $C^{\infty}\left(\boldsymbol{R}^{2(n-1)}\right)(k \rightarrow \infty)$ for any fixed $\theta,\left(x, \xi^{\prime}\right)$ Lebesgue dominated convergence theorem shows the first assertion of (2), and the second one follows from (1).

To show (1) we use the following lemma which is Lemma 4.7 in [3].

Lemma 5.3. Let $\Phi, \varphi$ be positive continuous functions on $\boldsymbol{R}^{n} \times \boldsymbol{R}^{n}$ satisfying the following condition (i) (iv) with some positive constants $C, c, \varepsilon, C^{\prime}$ : 
(i) $\quad c \leq \Phi(x, \xi) \leq C(1+|\xi|), \quad C \geq \varphi(x, \xi) \geq c(1+|\xi|)^{\varepsilon-1}$;

(ii) $\Phi(x, \xi) \varphi(x, \xi) \geq c$;

(iii) for any $R>1$ there exists $M>1$ such that $R^{-1} \leq \frac{1+|\xi|}{1+|\eta|} \leq R$ implies that $M(R)^{-1} \leq \frac{\Phi(x, \xi)}{\varphi(x, \xi)}\left(\frac{\Phi(y, \eta)}{\varphi(y, \eta)}\right)^{-1} \leq M(R)$

(iv) $C^{\prime-1} \leq \frac{\Phi(x, \xi)}{\Phi(y, \eta)} \leq C^{\prime}, C^{\prime-1} \leq \frac{\varphi(x, \xi)}{\varphi(y, \eta)} \leq C^{\prime}$ whenever $(y, \eta) \in U(x, \xi)=\{(y, \eta)$ $\left.\in \mathbb{R}^{n} \times \mathbb{R}^{n} ;|y-x|<\varphi(x, \xi),|\eta-\xi|<\Phi(x, \xi)\right\}$.

Let $b(x, y, \xi, \eta)$ be $a \mathbb{C}^{\infty}$-function in $(y, \eta)$ for any fixed $(x, \xi)$ satisfying the estimates

$$
\begin{aligned}
& \left|\partial_{y}^{\alpha} \partial_{\eta}^{\beta} b(x, y, \xi, \eta)\right| \leq C_{\alpha \beta} \sum_{\substack{r_{j} \in \mathbb{Z}_{+} \\
r_{1}+\cdots+r_{r}=\left|\beta_{\mid}\right| \\
r_{k+1}+\cdots+r_{k+q}=|\alpha|}} \prod_{j=1}^{k} \sup _{Q \in \mathcal{K}}\left(\Phi^{M_{j}-r_{j}}(Q) \varphi^{m_{j}}(Q)\right) \\
& \times \prod_{j=k+1}^{k+l} \sup _{Q \in K}\left(\Phi^{M_{j}}(Q) \varphi^{m_{j}-r_{j}}(Q)\right)
\end{aligned}
$$

where $K=$ the covex hull of $\{(x, \xi),(x, \eta),(y, \xi),(y, \eta)\}, M_{j}, m_{j} \in \mathbb{R}, k, l \in \mathbb{N}$. Set

$$
\begin{gathered}
a_{\phi, \varphi}^{p, q}(x, y, \xi, \eta)=\sum_{\substack{\sum_{j} \in Z_{+} \\
r_{1}+\cdots+r_{k}=q \\
r_{k+1}+\cdots+r_{k+l}=p}} \prod_{j=1}^{k} \sup _{Q \in K^{\prime}}\left(\Phi^{M_{j}-r_{j}}(Q) \varphi^{m_{j}}(Q)\right) \\
\times \prod_{j=k+1}^{k+l} \sup _{Q \in K_{k}}\left(\Phi^{M_{j}}(Q) \varphi^{m_{j}-r_{j}}(Q)\right), \\
a(x, \xi)=O S-\iint e^{-i y \cdot \eta} b(x, y+x, \xi, \eta+\xi) d y d \eta
\end{gathered}
$$

Define for $j \in \mathbb{Z}_{+}$

$$
|b|_{j}^{\phi, \varphi}=\max _{|\alpha|+|\beta| \leq j} \sup _{(x, y, \xi, \eta)}\left[\left|\partial_{y}^{\alpha} \partial_{\eta}^{\beta} b\right|\left(a_{\phi, \varphi}^{|\alpha|,|\beta|}\right)^{-1}\right](x, y, \xi, \eta) .
$$

Then one can find $C_{0}>0$ and $L \in \mathbb{N}$ depending only on $C, c, \varepsilon, C^{\prime}, M(4), k, l$, and a permutation $\left(M_{1}, \cdots, M_{k+l}, m_{1}, \cdots, m_{k+l}\right)$ of $2(k+l)$ real numbers such that

$$
|a(x, \xi)| \leq C_{0}|b|_{L}^{\Phi, \varphi}\left(\Phi^{M_{1}+\cdots+M_{k+l}} \varphi^{m_{1}+\cdots+m_{k+l}}\right)(x, \xi) .
$$

For a proof of this lemma, see the appendix in [2]. This lemma will also be used in a later part of this paper.

Now we continue the proof of the claim. From (5.6) 


$$
\begin{array}{r}
\left|\partial_{y^{\prime}}^{\alpha} \partial_{\eta^{\prime}}^{\beta}\left[f_{\theta}[a, b]\left(\left(t, x^{\prime}\right), y^{\prime}-x^{\prime}, \xi^{\prime}, \eta,-\xi^{\prime}\right)\right]\right| \leq|a|_{|\beta| \beta \mid}^{M, m}|b|_{|\alpha|}^{\left.M^{\prime}\right|^{\prime \prime}} \sup _{Q \in \mathbb{K}}\left(\Phi_{t}^{M-|\beta|} \varphi_{t}^{m}\right)(Q) \\
\times \sup _{Q \in \mathbb{K}}\left(\Phi_{*}^{M^{\prime}} \varphi_{t}^{m^{\prime}-|\alpha|}\right)(Q),
\end{array}
$$

where $K=\operatorname{ch}\left\{\left(x^{\prime}, \xi^{\prime}\right),\left(x^{\prime}, \eta^{\prime}\right),\left(y^{\prime}, \xi^{\prime}\right),\left(y^{\prime}, \eta^{\prime}\right)\right\}$, and $\Phi_{t}, \varphi_{t}$ are as in Lemma 5.1 . A remark just above Lemma 5.1 implies that if we take $n-1, \Phi_{t}(\cdot), \varphi_{t}(\cdot)$ for $n$, $\Phi, \varphi$ in Lemma 5.3, the conditions (i) (iv) in this lemma holds with uniform constants $C, c, \varepsilon=\frac{1}{2}, C^{\prime}$ in $t$. Thus taking $k=l=1, M_{1}=M, m_{1}=m, M_{2}=M^{\prime}$, $m_{2}=m^{\prime}$ in Lemma 5.3 we see from (5.10) that there exist constants $C_{3}>0, A \in \mathbb{N}$ depending only on $\Phi, \varphi, M, m, M^{\prime}, m^{\prime}$ satisfying

$$
\left|h_{\theta}[a, b]\left(x, \xi^{\prime}\right)\right| \leq C_{3}|a|_{A}^{M, m}|b|_{A}^{M^{\prime}, m^{\prime}}\left(\Phi^{M+M^{\prime}} \varphi^{m+m^{\prime}}\right)\left(x, \xi^{\prime}\right) .
$$

In view of the estimate (5.7) Lebesgue dominated covergence theorem shows that

$$
\begin{aligned}
& \partial_{x_{j}} h_{\theta}[a, b]=h_{\theta}\left[\partial_{x_{j}} a, b\right]+h_{\theta}\left[a, \partial_{x_{j}} b\right], \\
& \partial_{\xi_{j}} h_{\theta}[a, b]=h_{\theta}\left[\partial_{\xi_{j}} a, b\right]+h_{\theta}\left[a, \partial_{\xi_{j}} b\right] .
\end{aligned}
$$

Thus we see by induction that

$$
\partial_{x}^{\alpha} \partial_{\xi^{\prime}}^{\beta} h_{\theta}[a, b]=\sum_{\substack{\nu \leq \alpha \\
\mu \leq \beta}}\left(\begin{array}{l}
\alpha \\
\nu
\end{array}\right)\left(\begin{array}{l}
\beta \\
\mu
\end{array}\right) h_{\theta}\left[\partial_{x}^{\alpha-\nu} \partial_{\xi^{\prime}}^{\beta}-\mu a, \partial_{x}^{\nu} \partial_{\xi^{\prime}}^{\mu}, b\right] .
$$

From (5.11) and (5.12) there exist constants $C_{4}>0$ and $B \in N$ depending only on $\Phi, \varphi, M, m, M^{\prime}, m^{\prime}, \alpha, \beta$ satisfying

$$
\left|\partial_{x}^{\alpha} \partial_{\xi^{\prime}}^{\beta} h_{\theta}[a, b]\left(x, \xi^{\prime}\right)\right| \leq C_{4}|a|_{B}^{M, m}|b|{ }_{B}^{M^{\prime}, m^{\prime}}\left(\Phi^{M+M^{\prime}-|\beta|} \varphi^{m+m^{\prime}-|\alpha|}\right)\left(x, \xi^{\prime}\right)
$$

Thus the assertion (1) has been proved.

Let us return to the proof of Lemma 5.2. $a \circ b \in S_{\Phi, \varphi}^{M+M M^{\prime}, m+m^{\prime}}$ follows from the fact that $a \circ b=h_{\theta}[a, b]$ when $\theta=1$ and the claim (1). We have that

$$
r_{N \theta}[a, b]=N \sum_{|\alpha|=N} \frac{1}{\alpha !} h_{\theta}\left[\partial_{\xi}^{\alpha}, a, D_{x^{\prime}}^{\alpha} b\right]
$$

Since $\partial_{\xi^{\prime}}^{\alpha} a \in S_{\Phi, \varphi}^{M-N, m}$ and $D_{x^{\prime}}^{\alpha} b \in S_{\Phi, \varphi}^{M^{\prime}, m^{\prime}-N}$ on the right hand side, the claim (1) shows that the assertion (5.5) holds. Choose $\chi \in C_{0}^{\infty}\left(\mathbb{R}^{n} \times \boldsymbol{R}^{n-1}\right)$ with $\chi(0,0)=1$ and set $a_{k}=\chi_{k} a, b_{k}=\chi_{k} b$ with $\chi_{k}\left(x, \xi^{\prime}\right)=\chi\left(\frac{x}{k}, \frac{\xi^{\prime}}{k}\right)$. Then $\left\{a_{k}\right\}_{k=1}^{\infty}$ and $\left\{b_{k}\right\}_{k=1}^{\infty}$ satisfy the conditions in the claim (2). This implies that for all $\alpha \in \mathbb{Z}_{+}$and $\theta \in[0,1]$

$$
h_{\theta}\left[\partial_{\xi^{\prime}}^{\alpha} a_{k}, D_{x^{\prime}}^{\alpha} b_{k}\right]\left(x, \xi^{\prime}\right) \rightarrow h_{\theta}\left[\partial_{\xi^{\prime}}^{\alpha} a, D_{x^{\prime}}^{\alpha} b\right]\left(x, \xi^{\prime}\right) \quad \text { (pointwise) }
$$


as $k \rightarrow \infty$ being bounded in $\theta$ for any fixed $\left(x, \xi^{\prime}\right)$.

Since $h_{\theta}[a, b]=a \circ b$ for $\theta=1$, this implies that $\lim _{k \rightarrow \infty}\left(a_{k} \circ b_{k}\right)\left(x, \xi^{\prime}\right)=(a \circ b)$ $\left(x, \xi^{\prime}\right)$ for any $\left(x, \xi^{\prime}\right)$. From (5.7) and $(5.9) h_{\theta}[a, b]\left(x, \xi^{\prime}\right)$ is continuous function in $\theta$ for any fixed $\left(x, \xi^{\prime}\right)$. Thus from (5.13) and Lebesgue dominated convergence theorem, the above convergence also shows that $\lim _{k \rightarrow \infty} r_{N}\left[a_{k}, b_{k}\right]$ $\left(x, \xi^{\prime}\right)=r_{N}[a, b]\left(x, \xi^{\prime}\right)$ for any $\left(x, \xi^{\prime}\right)$. Letting $k \rightarrow \infty$ in (5.2) with $a=a_{k}, b=b_{k}$ we see that (5.2) also holds for general $a, b$.

Finally we show that $(a \circ b)\left(x, D^{\prime}\right) u=a\left(x, D^{\prime}\right) b\left(x, D^{\prime}\right) u$ for all $u \in \mathcal{S}\left(\mathbb{R}^{n}\right)$. This is easily checked if $a, b \in C_{0}^{\infty}\left(\mathbb{R}^{n} \times \mathbb{R}^{n-1}\right)$. Taking $\theta=1, a=a_{k}, b=b_{k}$, we see that $\left\{a_{k} \circ b_{k}\right\}_{k=1}^{\infty}$ is bounded in $S_{\Phi, \varphi}^{M+M^{\prime}, m+m^{\prime}}$. Thus Lebesgue dominated convergence theorem shows that $\left(a_{k} \circ b_{k}\right)\left(x, D^{\prime}\right) u(x) \rightarrow(a \circ b)\left(x, D^{\prime}\right) u(x)$ pointwise as $k \rightarrow \infty$. Thus $\left(a_{k} \circ b_{k}\right)\left(x, D^{\prime}\right) u \rightarrow(a \circ b)\left(x, D^{\prime}\right) u$ in $\mathcal{S}\left(\mathbb{R}^{n}\right)$ since $\left\{\left(a_{k} \circ b_{k}\right)\left(x, D^{\prime}\right) u\right\}_{k=1}^{\infty}$ is bounded in $S\left(\mathbb{R}^{n}\right)$ and $S\left(\mathbb{R}^{n}\right)$ is a Montel space. On the other hand $\left(a_{k} \circ b_{k}\right)$ $\left(x, D^{\prime}\right) u-a\left(x, D^{\prime}\right) b\left(x, D^{\prime}\right) u=\left(a_{k}\left(x, D^{\prime}\right)-a\left(x, D^{\prime}\right)\right) b\left(x, D^{\prime}\right) u+a_{k}\left(x, D^{\prime}\right)\left(b_{k}\left(x, D^{\prime}\right)-\right.$ $\left.b\left(x, D^{\prime}\right)\right) u$, and Lebesgue's theorem shows that $a_{k}\left(x, D^{\prime}\right) u \rightarrow a\left(x, D^{\prime}\right) u$ and $b_{k}\left(x, D^{\prime}\right) u \rightarrow b\left(x, D^{\prime}\right) u$ for all $u \in S\left(\mathbb{R}^{n}\right)$. Thus on the right hand side of the above equality the first term converges to 0 in $S\left(\mathbb{R}^{n}\right)$ and the second term does also because $\left\{a_{k}\left(x, D^{\prime}\right)\right\}_{k=1}^{\infty}$ is equicontinuous in the set of all cotinuous linear operators on $S\left(\mathbb{R}^{n}\right)$ into itself. This completes the proof.

Lemma 5.4. Let $a \in S_{\Phi, \varphi}^{M, m}$ and set

$$
a^{\sharp}\left(x, \xi^{\prime}\right)=(2 \pi)^{-(n-1)} O S-\iint e^{-i\left(y^{\prime}-x^{\prime}\right) \cdot\left(\eta^{\prime}-\xi^{\prime}\right)} \overline{a\left(\left(x_{1}, y^{\prime}\right), \eta^{\prime}\right)} d y^{\prime} d \eta^{\prime} .
$$

Then we have that

$$
a^{\sharp}\left(x, \xi^{\prime}\right)=\sum_{|\alpha|<N} \frac{1}{\alpha !} \partial_{\xi^{\prime}}^{\alpha} D_{x^{\prime}}^{\alpha} \bar{a}\left(x, \xi^{\prime}\right)+r_{N}[a]\left(x, \xi^{\prime}\right)
$$

where

$$
\begin{gathered}
r_{N}[a]\left(x, \xi^{\prime}\right)=\int_{0}^{1} r_{N \theta}[a]\left(x, \xi^{\prime}\right)(1-\theta)^{N-1} d \theta, \\
r_{N \theta}[a]\left(x, \xi^{\prime}\right)=N \sum_{|\alpha|=N} \frac{1}{\alpha !}(2 \pi)^{-(n-1)} O S-\iint e^{-i\left(y^{\prime}-x^{\prime}\right) \cdot\left(\eta^{\prime}-\xi^{\prime}\right)} \\
\left(\partial_{\xi^{\prime}}^{\alpha} D_{x^{\prime}}^{\alpha} \bar{a}\right)\left(\left(x_{1}, y^{\prime}\right), \xi^{\prime}+\theta\left(\eta^{\prime}-\xi^{\prime}\right)\right) d y^{\prime} d \eta^{\prime}, \\
\left\{r_{N \theta}[a]\right\}_{\theta \in[0,1]} \text { is bounded in } S_{\phi, \varphi}^{M-N, m-N} .
\end{gathered}
$$

Moreover we have that

$\left(a\left(\left(t, x^{\prime}\right), D^{\prime}\right) u, v\right)=\left(u, a^{\mathbb{E}}\left(\left(t, x^{\prime}\right), D^{\prime}\right) v\right)$ for any $u, v \in \mathcal{S}\left(\mathbb{R}^{n}\right)$ and any fixed $t \in \mathbb{R}$ 
where (, ) is the inner product of $L^{2}\left(\boldsymbol{R}_{x^{\prime}}^{n-1}\right)$.

Proof. If $a \in C_{0}^{\infty}\left(\boldsymbol{R}^{n} \times \boldsymbol{R}^{n-1}\right)$, it is not difficult to check (5.15) using Taylor's formula and Fourier inversion formula. Now we set

$$
h_{\theta}[a]\left(x, \xi^{\prime}\right)=(2 \pi)^{-(n-1)} O S-\iint e^{-i y^{\prime} \cdot \eta^{\prime}} a\left(\left(x_{1}, y^{\prime}+x^{\prime}\right), \xi^{\prime}+\theta \eta^{\prime}\right) d y^{\prime} d \eta^{\prime}
$$

for $a \in S_{\Phi, \varphi}^{M, m}, \theta \in[0,1]$.

Claim. (1) $h_{\theta}[a] \in S_{\phi, \varphi}^{M, m}$ and for any $L \in \mathbf{N}$ there exist $C>0$ and $P \in \boldsymbol{N}$ depending only on $\Phi, \varphi, M, m, L$ such that

$$
\sup _{\theta \in[0,1]}\left|\partial_{x}^{\alpha} \partial_{\xi^{\prime}}^{\beta} h_{\theta}[a]\left(x, \xi^{\prime}\right)\right| \leq C|a|_{P}^{M, m} \Phi^{M-|\beta|}\left(x, \xi^{\prime}\right) \varphi^{m-|\alpha|}\left(x, \xi^{\prime}\right)
$$

for all $\alpha, \beta$ with $|\alpha|+|\beta| \leq L$.

(2) If $\left\{a_{k}\right\}_{k=1}^{\infty}$ be a bounded set in $S_{\phi, \varphi}^{M, m}$ with $a_{k} \rightarrow a$ in $C^{\infty}\left(\boldsymbol{R}^{n} \times \boldsymbol{R}^{n-1}\right)$, $\left\{h_{\theta}\left[a_{k}\right]\right\}_{\theta \in[0,1]}^{k \in N}$ is bounded set in $S_{\Phi, \varphi}^{M, m}$ and $h_{\theta}\left[a_{k}\right]\left(x, \xi^{\prime}\right) \rightarrow h_{\theta}[a]\left(x, \xi^{\prime}\right)$ for any $\left(x, \xi^{\prime}\right)$ and $\theta$.

Proof of claim. We show (1) first. We set

$$
f_{\theta}[a]\left(x, y^{\prime}, \xi^{\prime}, \eta^{\prime}\right)=a\left(\left(x_{1}, y^{\prime}+x^{\prime}\right), \xi^{\prime}+\theta \eta^{\prime}\right) .
$$

Then we have that

$$
\begin{aligned}
& \left|\partial_{y^{\prime}}^{\alpha} \partial_{\eta^{\prime}}^{\beta} f_{\theta}[a]\left(x, y^{\prime}, \xi^{\prime}, \eta^{\prime}\right)\right| \leq|a|_{\left|\alpha^{\prime}\right|+|\beta|}^{M, m} \Phi^{M-|\beta|}\left(\left(x_{1}, y^{\prime}+x^{\prime}\right), \xi^{\prime}+\theta \eta^{\prime}\right) \\
& \times \varphi^{m-|\alpha|}\left(\left(x_{1}, y^{\prime}+x^{\prime}\right), \xi^{\prime}+\theta \eta^{\prime}\right) \\
& \leq C_{1}|a|_{\left|\alpha^{\prime}\right|+|\beta|}^{M, m}\left\langle\xi^{\prime}\right\rangle^{|M|+(|m|) / 2+(|\alpha|) / 2}\left\langle\eta^{\prime}\right\rangle^{|M|+(|m|) / 2+(|\alpha|) / 2} \text {, }
\end{aligned}
$$

where $C_{1}$ depends only on $\Phi, \varphi, M, m, \alpha, \beta$. We also have that for any $L, N$ $\in \mathbb{N}$

$$
\begin{aligned}
& \left.\left|\left\langle y^{\prime}\right\rangle^{-2 L}\left(1-\Delta_{\eta^{\prime}}\right)^{L}\right|\left\langle\eta^{\prime}\right\rangle^{-2 N}\left(1-\Delta_{y^{\prime}}\right)^{N} f_{\theta}[a]\left(x, y^{\prime}, \xi^{\prime}, \eta^{\prime}\right)\right] \mid \\
& \quad \leq C_{2}|a|_{2(N+L)}^{M, m}\left\langle\xi^{\prime}\right\rangle^{|M|+(|m|) / 2+N}\left\langle\eta^{\prime}\right\rangle^{|M|+(|m|) / 2-N}\left\langle y^{\prime}\right\rangle^{-2 L}
\end{aligned}
$$

where $C_{2}$ depends only on $\Phi, \varphi, M, m, L, N$. Thus, when $L, N \in N$ satisfy

$$
|M|+\frac{|m|}{2}-N<-(n-1), \quad-2 L<-(n-1)
$$

we have that

$$
\begin{aligned}
& h_{\theta}[a]\left(x, \xi^{\prime}\right)=(2 \pi)^{-(n-1)} \iint e^{-i y^{\prime} \cdot \eta^{\prime}}\left\langle y^{\prime}\right\rangle^{-2 L} \\
& \quad \times\left(1-\Delta_{\eta^{\prime}}\right)^{L}\left[\left\langle\eta^{\prime}\right\rangle^{-2 N}\left(1-\Delta_{y^{\prime}}\right)^{N} f_{\theta}[a]\left(x, y^{\prime}, \xi^{\prime}, \eta^{\prime}\right)\right] d y^{\prime} d \eta^{\prime} .
\end{aligned}
$$


From (5.21) and Lemma 5.3 we obtain the estimates (5.20) for $L=0$. Using the estimate (5.22) for first derivatives of $a$ and Lebesgue dominated convergence theorem we see that $\partial_{x_{j}} h_{\theta}[a]=h_{\theta}\left[\partial_{x_{j}} a\right], \partial_{\xi_{j}} h_{\theta}[a]=h_{\theta}\left[\partial_{\xi_{j}} a\right]$. Thus by induction we have that

$$
\partial_{x}^{\alpha} \partial_{\xi^{\prime}}^{\beta} h_{\theta}[a]=h_{\theta}\left[\partial_{x}^{\alpha} \partial_{\xi^{\prime}}^{\beta} a\right] .
$$

Thus from case that $L=0$ in (5.20) we also obtain the estimates (5.20) for all $L$.

Next we show (2). From the estimate (5.22) with $a_{k}$ for $a$ and (5.23) Lebesgue's theorem implies the second statement in (2) and the first one follows from (1). This completes the proof of the claim.

Now we return to the proof of the lemma. $a^{\sharp} \in S_{\Phi, \varphi}^{M, m}$ follows from the fact that $a^{\sharp}=h_{\theta}[\bar{a}]$ when $\theta=1$ and the claim (1). The boundedness of $\left\{r_{N \theta}\right\}_{\theta \in[0,1]}$ in $S_{\Phi, \varphi}^{M-N, m-N}$ follows from (5.24), because

$$
r_{N \theta}[a]=N \sum_{|\alpha|=N} \frac{1}{\alpha !} h_{\theta}\left[\partial_{\xi^{\prime}}^{\alpha} D_{x^{\prime}}^{\alpha} \bar{a}\right]
$$

Note that $h_{\theta}[a]\left(x, \xi^{\prime}\right)$ is a continuous function in $\theta$ for any fixed $\left(x, \xi^{\prime}\right)$ from (5.23). Take $\left\{a_{k}\right\}_{k=1}^{\infty} \subset C_{0}^{\infty}\left(\mathbb{R}^{n} \times \mathbb{R}^{n-1}\right)$ with the properties in the claim (2) as in the proof of Lemma 5.2. (5.24) and the claim (2) imply $\lim _{k \rightarrow \infty} r_{N}\left[a_{k}\right]\left(x, \xi^{\prime}\right)$ $=r_{N}[a]\left(x, \xi^{\prime}\right)$ pointwise from Lebesgue's theorem. Thus letting $k \rightarrow \infty$ in (5.15) with $a_{k}$ for $a$ we obtain (5.15) for a general $a \in S_{\Phi, \varphi}^{M, m}$. Finally we show (5.19). When $a \in C_{0}^{\infty}\left(\mathbb{R}^{n} \times \mathbb{R}^{n-1}\right)$, this can be easily checked. Note that $\left\{a_{k}^{\infty}\right\}_{k=1}^{\infty}$ is bounded in $S_{\Phi, \varphi}^{M, n}$ and $\lim _{k \rightarrow \infty} a_{k}^{*}\left(x, \xi^{\prime}\right)=a^{\sharp}\left(x, \xi^{\prime}\right)$ pointwise from the claim (2). Thus noting Lebesgue's theorem and letting $k \rightarrow \infty$ in (5.19) with $a_{k}$ for $a$ we obtain (5.19) for general $a \in S_{\Phi, \varphi}^{M, m}$. This completes the proof.

Let $q(x, \xi) \in C^{\infty}\left(\mathbb{R}^{n} \times \mathbb{R}^{n}\right)$ such that $q(x, \xi)=\sum_{j=0}^{m} a_{j}\left(x, \xi^{\prime}\right) \xi_{1}^{j}$ with $a_{j} \in$ $\cup(P, p) \in \mathbb{R}^{2} S_{\Phi, \varphi}^{p, p}$ and $a_{m} \neq 0$. This expression is clearly unique. Then we define an operator $q(x, D)$ from $S\left(\mathbb{R}^{n}\right)$ to $\mathcal{S}\left(\mathbb{R}^{n}\right)$ by

$$
q(x, D) u=\sum_{j=0}^{m} a_{j}\left(x, D^{\prime}\right) D_{1}^{j} u, \quad u \in \mathcal{S}\left(\mathbb{R}^{n}\right) .
$$

$q(x, D)$ maps $\mathcal{S}_{T}\left(\mathbb{R}^{n}\right)$ into $\mathcal{S}_{T}\left(\mathbb{R}^{n}\right)$.

Lemma 5.5. Let $q_{i}(x, D)=\sum_{j=0}^{m} a_{i j}\left(x, D^{\prime}\right) D_{1}^{j}(i=1,2)$ be operators defined as above. Then

$$
\left(q_{1} \circ q_{2}\right)(x, D)=q_{1}(x, D) q_{2}(x, D)
$$


with

$$
\begin{aligned}
\left(q_{1} \circ q_{2}\right)(x, D)=\sum_{j=0}^{m_{1}} \frac{(2 \pi)^{-(n-1)}}{j !} O S-\int & \int e^{-i y^{\prime} \cdot \eta^{\prime}}\left(\partial_{\xi_{1}}^{j} q_{1}\right)\left(x, \xi+\left(0, \eta^{\prime}\right)\right) \\
& \times\left(D_{x_{1}}^{j} q_{2}\right)\left(\left(x_{1}, y^{\prime}+x^{\prime}\right), \xi\right) d y^{\prime} d \eta^{\prime}
\end{aligned}
$$

and for any $\left(N_{0}, \cdots, N_{m_{1}}\right) \in N^{m_{1}+1}$ with $N_{j} \leq 1$ we have that

$$
\begin{aligned}
\left(q_{1} \circ q_{2}\right)(x, \xi)= & \sum_{j=0}^{m_{1}} \frac{1}{j !}\left\{\sum_{|\alpha|<N_{j}} \frac{1}{\alpha !}\left(\partial_{\xi_{1}}^{j} \partial_{\xi^{\prime}}^{\alpha} q_{1}\right)(x, \xi)\left(D_{x_{1}}^{j} D_{x^{\prime}}^{\alpha} q_{2}\right)(x, \xi)+r_{j}(x, \xi)\right\}, \\
r_{j}(x, \xi)= & \int_{0}^{1} r_{j \theta}(x, \xi)(1-\theta)^{N_{j}-1} d \theta, \\
r_{j \theta}(x, \xi)= & N_{j} \sum_{|\alpha|=N_{j}} \frac{(2 \pi)^{-(n-1)}}{\alpha !} O S-\iint e^{-i y^{\prime} \cdot \eta^{\prime}}\left(\partial_{\xi_{1}}^{j} \partial_{\xi^{\prime}}^{\alpha} q_{1}\right)\left(x, \xi+\theta\left(0, \eta^{\prime}\right)\right) \\
& \times\left(D_{x_{1}}^{j} D_{x^{\prime}}^{\alpha} q_{2}\right)\left(\left(x_{1}, y^{\prime}+x^{\prime}\right), \xi\right) d y^{\prime} d \eta^{\prime} .
\end{aligned}
$$

Proof. When $q_{i}(x, \xi)$ are monomials in $\xi_{1}$, the result follows from Lemma 5.2 and Leibniz rule. The general case follows from bilinearlity of (5.25), (5.26). The results in the remaining part of this section are used to prove Lemmas 3.7 and 3.10 .

Lemma 5.6. Let $q \in C^{\infty}\left(\mathbb{R}^{n} \times \mathbb{R}^{n}\right)$ be as before Lemma 5.5, and let $a \in$ $S_{\Phi, \varphi}^{M, m}$. Let $N \in N$. Then there exist sets of constants $\left\{C_{\beta}\right\}_{\beta \in Z_{+}^{n-1}}$ and $\left\{C_{\alpha l}\right\}_{(\alpha, l) \in Z^{n-1} \times Z_{+}}$depending only on $N$ such that $|\beta|<N$

$$
\begin{aligned}
&(a \circ q)(x, \xi)=a\left(x, \xi^{\prime}\right) q(x, \xi)+\sum_{0<|\alpha|<N} C_{\alpha}\left(\partial_{\xi^{\prime}}^{\alpha} a \circ D_{x}^{\alpha} q\right)(x, \xi) \\
&+\sum_{|\alpha|=N} \int_{0}^{1} q_{\alpha}(\theta, x, \xi) \sum_{l \leq N-1} C_{\alpha l}(1-\theta)^{N-1-l} d \theta, \\
& q_{\alpha}(\theta, x, \xi)=(2 \pi)^{-(n-1)} O S-\iint e^{-i y^{\prime} \circ \eta^{\prime}} \partial_{\xi^{\prime}}^{\alpha} a\left(x, \xi^{\prime}+\theta \eta^{\prime}\right) D_{x^{\prime}}^{\alpha} q\left(\left(x_{1}, y^{\prime}\right.\right. \\
&\left.\left.+x^{\prime}\right), \xi\right) d y^{\prime} d \eta^{\prime} .
\end{aligned}
$$

Proof. It suffices to prove by induction on $K, 0 \leq K<N$ that

$$
\begin{aligned}
(a \circ q)(x, \xi)=a\left(x, \xi^{\prime}\right) q(x, \xi) & +\sum_{0<|\alpha| \leq K} C_{K \alpha}\left(\partial_{\xi^{\prime}}^{\alpha} a \circ D_{x^{\prime}}^{\alpha} q\right)(x, \xi) \\
& +\sum_{K<|\alpha|<N} C_{K \alpha} \partial_{\xi^{\prime}}^{\alpha} a\left(x, \xi^{\prime}\right) D_{x^{\prime}}^{\alpha} q(x, \xi) \\
& +\sum_{|\alpha|=N} \int_{0}^{1} q_{\alpha}(\theta, x, \xi) \sum_{l \leq K} C_{K \alpha l}(1-\theta)^{N-1-l} d \theta .
\end{aligned}
$$

This is trivial for $K=0$ and we assume that this is true for $K-1$. When $|\alpha|=K$, we have 


$$
\begin{aligned}
\partial_{\xi^{\prime}}^{\alpha} a\left(x, \xi^{\prime}\right) D_{x}^{\alpha} q(x, \xi) & =\left(\partial_{\xi^{\prime}}^{\alpha} a \circ D_{x}^{\alpha} q\right)(x, \xi)-\sum_{0<|\beta|<N-K} \frac{1}{\beta !} \partial_{\xi^{\prime}}^{\alpha+\beta} a\left(x, \xi^{\prime}\right) D_{x^{\prime}}^{\alpha+\beta} q(x, \xi) \\
& -(N-k) \sum_{|\beta|=N-K_{0}} \frac{1}{\beta !} \int_{0}^{1} q_{\alpha+\beta}(\theta, x, \xi)(1-\theta)^{N-K-1} d \theta
\end{aligned}
$$

Substituting this into the equality for $K-1$ we get one for $K$. This completes the proof.

Lemma 5.7. Let $q(x, \xi)=\sum_{j=0}^{s} a_{j}\left(x, \xi^{\prime}\right) \xi_{1}^{j}, a_{j} \in U_{(P, p) \in R^{2}} S_{\Phi, \varphi}^{P, p}$, and let $a \in$ $S_{\phi, \varphi}^{M, m}$. Let $N, L \in \mathbb{N}$ with $1 \leq L<N, s<N$. Then we have that

$$
\begin{aligned}
& (q \circ a)(x, \xi)=q(x, \xi) a\left(x, \xi^{\prime}\right)+\sum_{\substack{0 \leq j \leq s \\
0<|\alpha|+j<L}} \frac{1}{\alpha ! j !} \partial_{\xi^{\prime}}^{\alpha} \partial_{\xi_{1}}^{j} q(x, \xi) D_{x^{\prime}}^{\alpha} D_{x_{1}}^{j} a\left(x, \xi^{\prime}\right) \\
& +\sum_{\substack{0 \leq j \leq s \\
L \leq|\alpha|+j<N}} \sum_{|\beta|<N-|\alpha|-j} C_{\alpha \beta j}\left(\partial_{\xi \prime}^{\beta} D_{x^{\prime}}^{\alpha} D_{x_{1}}^{j} a \circ D_{x^{\prime}}^{\beta} \partial_{\xi^{\prime}}^{\alpha}, \partial_{\xi_{1}}^{j} q\right)(x, \xi) \\
& +\sum_{\substack{0 \leq j \leq s \\
|\alpha|+j=N N}} \frac{N-j}{\alpha ! j !} \int_{0}^{1} q_{\alpha j}(\theta, x, \xi)(1-\theta)^{N-j-1} d \theta \\
& +\sum_{\substack{0 \leq j \leq s \\
L \leq|\alpha|+j<N}} \sum_{|\beta|=N-|\alpha|-j} \int_{0}^{1} q_{\alpha \beta j}(\theta, x, \xi) \sum_{l \leq|\beta|-1} C_{\alpha \beta j l}(1-\theta)^{|\beta|-1-l} d \theta
\end{aligned}
$$

where $C_{\alpha \beta j}, C_{\alpha \beta j l}$ are constants depending only on its suffixes and $N, L$, and

$$
\begin{gathered}
q_{\alpha j}=(2 \pi)^{-(n-1)} \iint e^{-i y^{\prime} \cdot \eta^{\prime}} \partial_{\xi^{\prime}}^{\alpha} \partial_{\xi_{1}}^{j} q\left(x, \xi+\theta\left(0, \eta^{\prime}\right)\right) D_{x^{\prime}}^{\alpha} D_{x_{1}}^{j} a\left(\left(x_{1}, y^{\prime}+x^{\prime}\right), \xi^{\prime}\right) d y^{\prime} d \eta^{\prime}, \\
q_{\alpha \beta j}=(2 \pi)^{-(z-1)} \iint e^{-i y^{\prime} \cdot \eta^{\prime}} \partial_{\xi^{\prime}}^{\beta} D_{x^{\prime}}^{\alpha} D_{x_{1}}^{j} a\left(x, \xi^{\prime}+\theta \eta^{\prime}\right)\left(D_{x^{\prime}}^{\beta} \partial_{\xi^{\prime}}^{\alpha} \partial_{\xi_{1}}^{j} q\right)\left(\left(x_{1}, y^{\prime}\right.\right. \\
\left.\left.+x^{\prime}\right), \xi\right) d y^{\prime} d \eta^{\prime} .
\end{gathered}
$$

Proof. By Lemma 5.5

$$
\begin{aligned}
(q \circ a)(x, \xi)= & \sum_{\substack{0 \leq j \leq s \\
|\alpha|+j<N}} \frac{1}{\alpha ! j !} \partial_{\xi^{\prime}}^{\alpha} \partial_{\xi_{1}}^{j} q(x, \xi) D_{x^{\prime}}^{\alpha} D_{x_{1}}^{j} a\left(x, \xi^{\prime}\right) \\
& +\sum_{\substack{0 \leq j \leq s \\
|\alpha|+j=N}} \frac{N-j}{\alpha ! j !} \int_{0}^{1} q_{\alpha j}(\theta, x, \xi)(1-\theta)^{N-j-1} d \theta .
\end{aligned}
$$

Applying Lemma 5.6 with $D_{x^{\prime}}^{\alpha \prime \prime} D_{x_{1}}^{j} a, \partial_{\xi^{\prime}}^{\alpha} \partial_{\xi_{1}}^{j} q$ with $L \leq|\alpha|+j<N$, and $N-$ $|\alpha|-j$ for $a, q, N$ we have

$$
\begin{aligned}
\partial_{\xi^{\prime}}^{\alpha} \partial_{\xi_{1}}^{j} q(x, \xi) D_{x^{\prime}}^{\alpha} D_{x_{1}}^{j} a\left(x, \xi^{\prime}\right)= & \left(D_{x^{\prime}}^{\alpha} D_{x_{1}}^{j} a \circ \partial_{\xi^{\prime}}^{\alpha} \partial_{\xi^{\prime} 1}^{j} q\right)(x, \xi) \\
& +\sum_{0<|\beta|<N-|\alpha|-j} C_{\alpha \beta_{j}}\left(\partial_{\xi^{\prime}}^{\beta} D_{x^{\prime}}^{\alpha} D_{x_{1}}^{j} a \circ D_{x^{\prime}}^{\beta} \partial_{\xi^{\prime}}^{\alpha} \partial_{\xi_{1}}^{j} q\right)(x, \xi)
\end{aligned}
$$




$$
+\sum_{|\beta|=N-|\alpha|-j} \int_{0}^{1} q_{\alpha \beta j}(\theta, x, \xi) \sum_{l=0}^{|\beta|-1} C_{\alpha \beta j l}(1-\theta)^{|\beta|-1-l} d \theta
$$

Substituting these equalities into (5.27) we get the second equality. This completes the proof.

Lemma 5.8. Let $q(x, \xi)=\sum_{j=0}^{3} a_{j}\left(x, \xi^{\prime}\right) \xi_{1}^{j}$ with $a_{3}=$ constant and $a_{j} \in$ $S_{1,0}^{m_{j}}\left(\boldsymbol{R}^{n} \times \boldsymbol{R}^{n-1}\right)$ for $j \leq 2$, and set $m_{3}=0$. Let $a \in S_{\Phi, \varphi}^{M, m}$, and set for $\alpha, \beta \in \mathbb{Z}_{+}^{n}$

$$
\begin{aligned}
{[a, q]_{\alpha \beta}(\theta, x, \xi)=(2 \pi)^{-(n-1)} O S-\iint e^{-i y^{\prime} \cdot \eta^{\prime}} a\left(x, \xi^{\prime}+\theta \eta^{\prime}\right) } & \\
& \times\left(\partial_{\xi}^{\alpha} \partial_{x}^{\beta} q\right)\left(\left(x_{1}, y^{\prime}+x^{\prime}\right), \xi\right) d y^{\prime} d \eta^{\prime}, \quad \theta \in[0,1] .
\end{aligned}
$$

Then if $\alpha_{1} \leq 3$ and $|\alpha|+|\beta|>0$, we have that

$$
[a, q]_{\alpha \beta}(\theta, x, \xi)=\sum_{j=0}^{\min \left(2,3-\alpha_{1}\right)} b_{j \theta}\left(x, \xi^{\prime}\right) \xi_{1}^{j}
$$

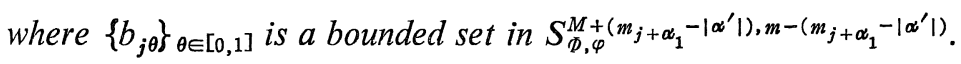

Proof. With a notation in the proof of Lemma 5.2 we have that when $\alpha_{1}$ $\leq 3$

$$
[a, q]_{\alpha \beta}(\theta, x, \xi)=\sum_{j=\alpha_{1}}^{3} \frac{j !}{\left(j-\alpha_{1}\right) !} h_{\theta}\left[a, \partial_{\xi^{\prime}}^{\alpha^{\prime}} \partial_{x}^{\beta} a_{j}\right]\left(x, \xi^{\prime}\right) \xi_{1}^{j-\alpha_{1}} .
$$

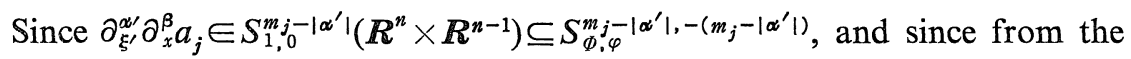
assumption that $a_{3}=$ constant the term for $j=3$ is dropped if $\alpha_{1}=0$ and $|\alpha|+$ $|\beta|>0$, the assertion follows from the claim (1) in the proof of Lemma 5.2. The proof is complete.

Corollary 5.1. Let $q$ be as in the above lemma with $m_{j}=3-j$, and let $a \in S_{1 / 2,1 / 2}^{l+(|\alpha|-|\beta|) / 2}\left(\mathbb{R}^{n} \times \boldsymbol{R}^{n-1}\right)$ with $\alpha, \beta \in \mathbb{Z}_{+}^{n}$ satisfying the same assumption as above. Then (5.28) holds with $\left\{b_{j \theta}\right\}_{\theta \in[0,1]}$ bounded in $S_{1 / 2,1 / 2}^{l+3-j-(|\alpha|+|\beta|) / 2}\left(\boldsymbol{R}^{n} \times \boldsymbol{R}^{n-1}\right)$.

Proof. Let $\Phi\left(x, \xi^{\prime}\right)=\left\langle\xi^{\prime}\right\rangle^{1 / 2}, \varphi\left(x, \xi^{\prime}\right)=\left\langle\xi^{\prime}\right\rangle^{-1 / 2}, M=-m=l+\frac{|\alpha|-|\beta|}{2}$. Then $a \in S_{\Phi, \varphi}^{M, m}$, and $M+m_{j+\alpha_{1}}-\left|\alpha^{\prime}\right|=l+3-j-\frac{|\alpha|+|\beta|}{2}$. We apply Lemma 5.8. Since

$$
\begin{aligned}
S_{\Phi, \varphi}^{M+\left(m_{j+\alpha_{1}}-\left|\alpha^{\prime}\right|\right), m-\left(m_{j+\alpha_{1}}-\left|\alpha^{\prime}\right|\right)} & =S_{\Phi, \varphi}^{M+m_{j+\alpha_{1}}-\left|\alpha^{\prime}\right|,-\left(M+m_{j+\alpha_{1}}-\left|\alpha^{\prime}\right|\right)} \\
& =S_{1 / 2,1 / 2}^{M+m_{j}-\alpha_{1}-\left|\alpha^{\prime}\right|}\left(\mathbb{R}^{n} \times \mathbb{R}^{n-1}\right)
\end{aligned}
$$

the conclusion follows. The proof is complete.

Lemma 5.9. Let $q$ and $a$ be as in Lemma 5.8, and set for $\alpha, \beta \in \mathbb{Z}_{+}^{n}$ 


$$
\begin{aligned}
{[q, a]_{\alpha \beta}(\theta, x, \xi)=(2 \pi)^{-(n-1)} O S-\iint } & e^{-i y^{\prime} \cdot \eta^{\prime}}\left(\partial_{\xi}^{\alpha} \partial_{x}^{\beta} q\right)\left(x, \xi+\theta\left(0, \eta^{\prime}\right)\right) \\
& \times a\left(\left(x_{1}, y^{\prime}+x^{\prime}\right), \xi^{\prime}\right) d y^{\prime} d \eta^{\prime}, \quad \theta \in[0,1] .
\end{aligned}
$$

Then if $\alpha_{1} \leq 3$ and $|\alpha|+|\beta|>0$, we have that

$$
[q, a]_{\alpha \beta}(\theta, x, \xi)=\sum_{j=0}^{\min \left(2,3-a_{1}\right)} b_{j \theta}\left(x, \xi^{\prime}\right) \xi_{1}^{j}
$$

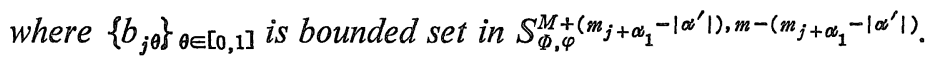

Proof. With a notation in the proof of Iemma 5.2 we have that if $\alpha_{1} \leq 3$.

$$
[q, a]_{\alpha \beta}(\theta, x, \xi)=\sum_{j=\alpha_{1}}^{3} \frac{j !}{(j-\alpha) !} h_{\theta}\left[\partial_{\xi^{\prime}}^{\alpha \prime} \partial_{x}^{\beta} a_{j}, a\right]\left(x, \xi^{\prime}\right) \xi_{1}^{j} .
$$

Now the proof is similar to that of Lemma 5.8. The proof is complete.

Corollary 5.2. Under the same assumption as in Corollary 5.1, (5.29) holds with $\left\{b_{j \theta}\right\}_{\theta \in[0,1]}$ bounded in $S_{1 / 2,1 / 2}^{l+3-(|\alpha|+|\beta|) / 2}\left(\mathbb{R}^{n} \times \mathbb{R}^{n-1}\right)$.

Proof. This is proved in the same way as in the proof of Corollary 5.1 from the above lemma. The proof is complete.

\section{§6. Proof of Proposition 3.2}

Proof of (1). Set $v=e^{1 / 2 \tau\left(x_{1}-T\right)^{2}} u$. Then

$$
e^{1 / 2 \tau\left(x_{1}-T\right)^{2}} L(x, D) u=\left(L(x, D)+i \tau\left(x_{1}-T\right)\right) v .
$$

Set

$$
\begin{gathered}
A_{1}=\operatorname{Re}(a+b)\left(x, D^{\prime}\right), \quad A_{2}=\operatorname{Im}(a+b)\left(x, D^{\prime}\right), \\
L_{1}=D_{1}-A_{1}, \quad L_{2}=A_{2}+\tau\left(x_{1}-T\right) .
\end{gathered}
$$

Then we have

$$
\left(\|L(x, D) u\|_{T}^{(\tau)}\right)^{2}=\left\|L_{1} v\right\|^{2}+2 \operatorname{Im}\left(L_{1} v, L_{2} v\right)+\left\|L_{2} v\right\|^{2},
$$

and

$$
\begin{aligned}
2 \operatorname{Im}\left(L_{1} v, L_{2} v\right)= & \tau\|v\|^{2}+\frac{1}{i}\left\{\left(v,\left[D_{1}, A_{2}\right] v\right)+\left(\left(A_{1}^{*}-A_{1}\right) v, L_{2} v\right)\right. \\
& \left.-\left(v,\left[A_{1}, A_{2}\right] v\right)+\left(\left(A_{2}^{*}-A_{2}\right) v, L_{1} v\right)\right\} \\
= & I+\cdots+V .
\end{aligned}
$$

From the proof of Lemma 5.4 with a notation in it we have

$$
[\operatorname{Re}(a+b)]^{\sharp}\left(x, \xi^{\prime}\right)-\operatorname{Re}(a+b)\left(x, \xi^{\prime}\right)=\int_{0}^{1} h_{\theta}\left[\sum_{|\alpha|=1} \partial_{\xi^{\prime}}^{\alpha} D_{x^{\prime}}^{\alpha} \operatorname{Re}(a+b)\right]\left(x, \xi^{\prime}\right) d \theta .
$$


Since $\partial_{\xi^{\prime}}^{\alpha} D_{x^{\prime}}^{\alpha} \operatorname{Re}(a+b) \in S_{\Phi, \varphi}^{0,{ }^{-1}}$ when $|\alpha|=1, A_{1}^{*}-A_{1} \in O p S_{\Phi, \varphi}^{0,-1}$ from the claim (1) in Lemma 5.4. Similarly we have $A_{2}^{*}-A_{2} \in O p S_{\Phi,}^{0,}{ }^{-1}$. Thus from (3.6) and Lemma 3.1 we have

$$
|I I I|+|V| \leq C_{1}\left\|E_{1 / 2} v\right\|\left(\left\|L_{1} v\right\|+\left\|L_{2} v\right\|\right) .
$$

From the proof of Lemma 5.2 with a notation in it we have

$$
\begin{aligned}
& (\operatorname{Re}(a+b) \circ \operatorname{Im}(a+b)-\operatorname{Im}(a+b) \circ \operatorname{Re}(a+b))\left(x, \xi^{\prime}\right) \\
& =\int_{0}^{1} \sum_{|\alpha|=1}\left(h_{\theta}\left[\partial_{\xi^{\prime}}^{\alpha} \operatorname{Re}(a+b), D_{x^{\prime}}^{\alpha} \operatorname{Im}(a+b)\right]-h_{\theta}\left[\partial_{\xi^{\prime}}^{\alpha} \operatorname{Im}(a+b), D_{x^{\prime}}^{\alpha} \operatorname{Re}(a+b)\right]\right)\left(x, \xi^{\prime}\right) d \theta .
\end{aligned}
$$

Thus the claim (1) in Lemma 5.2 shows $\left[A_{1}, A_{2}\right] \in O p S_{\Phi, \varphi}^{1,-1}$. Thus noting that $\left[D_{1}, A_{2}\right] \in O p S_{\mathscr{\phi}, \varphi}^{1,-1}$, we see that

$$
|I I|+|I V| \leq C_{2}\|v\| \mid\left\|E_{1} v\right\| .
$$

Since $\left\|E_{1 / 2} v\right\|^{2} \leq\left\|E_{1} v\right\|\|v\|$, applying (6.3) and (6.4) to (6.2) and using Schwartz inequality we see that

$$
2 \operatorname{Im}\left(L_{1} v, L_{2} v\right) \geq \tau\|v\|^{2}-\frac{1}{4}\left(\left\|L_{1} v\right\|^{2}+\left\|L_{2} v\right\|^{2}\right)-C_{3}\|v\|\left\|E_{1} v\right\|
$$

Now we shall use the assumption (3.3) of ellipticity to estimate the last term on the right of (6.5). To do so we prove

Lemma 6.1. Let $\lambda \in S_{\phi, \varphi}^{1,-1}$ with $\partial_{x_{j}} \lambda \in S_{\Phi, \varphi}^{1,-1}$ and $\partial_{\xi_{j}} \lambda \in S_{\phi, \varphi}^{0,0}$, and with $\inf _{\left|\xi^{\prime}\right| \geq R}\left|\lambda\left(x, \xi^{\prime}\right)\right| \Phi^{-1}\left(x, \xi^{\prime}\right) \varphi\left(x, \xi^{\prime}\right)>0$ for some $R>0$. Then there exists $\mu \in$ $S_{\Phi, \dot{\varphi}}^{-1,1}$ such that $\mu\left(x, \xi^{\prime}\right)=\frac{1}{\lambda\left(x, \xi^{\prime}\right)}$ when $\left|\xi^{\prime}\right|>2 R$ and $\mu \circ \lambda-1 \in S_{\bar{\Phi}, \dot{\varphi}}^{-1,1}$.

Proof. Let $\psi \in C^{\infty}\left(\boldsymbol{R}^{n-1}\right)$ with $\psi=1$ when $\left|\xi^{\prime}\right|>2, \psi=0$ when $\left|\xi^{\prime}\right|<1$. We define

$$
\mu\left(x, \xi^{\prime}\right)= \begin{cases}\frac{1}{\lambda\left(x, \xi^{\prime}\right)} \psi\left(R^{-1} \xi^{\prime}\right) & \left|\xi^{\prime}\right|>R \\ 0 & \left|\xi^{\prime}\right| \leq R .\end{cases}
$$

Then $\mu \in C^{\infty}\left(\boldsymbol{R}^{n} \times \boldsymbol{R}^{n-1}\right)$ and

$$
\left|\partial_{x}^{\alpha} \partial_{\xi^{\prime}}^{\beta} \mu\left(x, \xi^{\prime}\right)\right| \leq C_{\alpha \beta} \frac{1}{\left|\lambda\left(x, \xi^{\prime}\right)\right|}\left(\Phi^{-|\beta|} \varphi^{-|\alpha|}\right)\left(x, \xi^{\prime}\right) \quad \text { when } \quad\left|\xi^{\prime}\right|>R
$$

This implies $\mu \in S_{\Phi, \varphi}^{-1,1}$. Moreover since 


$$
\begin{aligned}
\partial_{\xi_{j}} \mu\left(x, \xi^{\prime}\right)=\frac{-\partial_{\xi_{j}} \lambda\left(x, \xi^{\prime}\right)}{\lambda\left(x, \xi^{\prime}\right)^{2}} \psi\left(R^{-1} \xi^{\prime}\right)+\frac{1}{\lambda\left(x, \xi^{\prime}\right)} R^{-1}\left(\partial_{\xi_{j}} \psi\right)\left(R^{-1} \xi^{\prime}\right) & \text { when }\left|\xi^{\prime}\right|>R,
\end{aligned}
$$

from $\partial_{\xi_{j}} \lambda \in S_{\phi, \varphi}^{0,0}$ and $\partial_{\xi_{j}} \psi \in C_{0}^{\infty}\left(\boldsymbol{R}^{n-1}\right)$ we see that

$$
\left|\partial_{x}^{\alpha} \partial_{\xi^{\prime}}^{\beta}\left(\partial_{\xi_{j}} \mu\left(x, \xi^{\prime}\right)\right)\right| \leq C_{\alpha \beta} \frac{1}{\left|\lambda\left(x, \xi^{\prime}\right)\right|^{2}}\left(\Phi^{-|\beta|} \varphi^{-|\alpha|}\right)\left(x, \xi^{\prime}\right) \text { when } \quad\left|\xi^{\prime}\right|>R .
$$

Thus $\partial_{\xi_{j}} \mu \in S_{\bar{\Phi}, \dot{\varphi}}^{-2,2}$. Thus from the proof of Lemma 5.2 with the notation in it we have

$$
(\mu \circ \lambda)\left(x, \xi^{\prime}\right)-(\mu \lambda)\left(x, \xi^{\prime}\right)=\int_{0}^{1} h_{\theta}[\mu, \lambda]\left(x, \xi^{\prime}\right) d \theta \in S_{\phi, \varphi^{-1}}^{-1} .
$$

Thus $\mu \circ \lambda-1 \in S_{\Phi, \dot{\varphi}}^{-1,1}$, for $\mu \lambda=1$ when $\left|\xi^{\prime}\right|>2 R$. This completes the proof.

From Lemma 6.1 there exists $b_{1} \in S_{\bar{\phi}, \dot{\varphi}}^{-1,1}$ with $b_{1} \circ \operatorname{Im}(a+b)-1 \in S_{\bar{\phi}, \dot{\varphi}}^{-1,1}$. Since $\left\langle\xi^{\prime}\right\rangle \in S_{\Phi, \varphi^{1}}^{1,-1}, b_{1} \circ \operatorname{Im}(a+b)-\left\langle\xi^{\prime}\right\rangle \in S_{\Phi_{, \varphi}^{0}, 0}$ with $b_{2}=\left\langle\xi^{\prime}\right\rangle \circ b_{1}$. Thus

$$
\begin{aligned}
\left\|E_{1} v\right\| & \leq C_{4}\left(\left\|A_{2} v\right\|+\|v\|\right) \\
& \leq C_{4}\left(\left\|L_{2} v\right\|+(\tau T+1)\|v\|\right) .
\end{aligned}
$$

Multiplying this inequality by $\|v\|$ and using Schwartz inequality we get that

$$
\|v\|\left\|E_{1} v\right\| \leq C_{5}(\tau T+1)\|v\|^{2}+\frac{1}{4}\left\|L_{2} v\right\|^{2}
$$

Substituting this into (6.5) we see that when $T+\frac{1}{\tau}<\delta$ for some $\delta>0$,

$$
2 \operatorname{Im}\left(L_{1} v, L_{2} v\right) \geq \frac{1}{2} \tau\|v\|^{2}-\frac{1}{2}\left(\left\|L_{1} v\right\|^{2}+\left\|L_{2} v\right\|^{2}\right) .
$$

Substituting this into (6.1) we get that when $T+\frac{1}{\tau}<\delta$,

$$
\|L(x, D) u\|_{T}^{(\tau)} \geq \frac{1}{\sqrt{6}}\left(\left\|L_{1} v\right\|+\left\|L_{2} v\right\|+\tau^{1 / 2}\|v\|\right)
$$

From (6.6) and (6.7) we have that when $T+\frac{1}{\tau}<\delta$,

$$
\left\|E_{1} u\right\|_{T}^{(\tau)}=\left\|E_{1} v\right\| \leq C_{5}\left(1+\tau^{1 / 2} T\right)\|L(x, D) u\|_{T}^{(\tau)} .
$$

Finally from inequalities that $\left\|D_{1} u\right\|_{T}^{(\tau)} \leq\|L(x, D) u\|_{T}^{(\tau)}+C_{6}\left\|E_{1} u\right\|_{T}^{(\tau)}$ and $T^{-1 / 2}\left\|E_{1 / 2} u\right\|_{T}^{(\tau)} \leq \frac{1}{\sqrt{2}}\left(\tau^{-1 / 2} T^{-1}\left\|E_{1} u\right\|_{T}^{(\tau)}+\tau^{1 / 2}\|u\|_{T}^{(\tau)}\right)$, this inequality and (6.7) im- 
ply that

$$
\tau^{-1 / 2} T^{-1}\left\|D_{1} u\right\|_{T}^{(\tau)}+T^{-1 / 2}\left\|E_{1 / 2} u\right\|_{T}^{(\tau)} \leq C_{7}\|L(x, D) u\|_{T}^{(\tau)}
$$

when $\tau^{1 / 2} T \geq 1$ and $T+\frac{1}{\tau}<\delta$. This completes the proof of (1).

Proof of (2). Let $\tau_{0}, T_{0}, C_{0}$ be constants as in Proposition 3.1(1), and assume that $\tau T^{2}>\tau_{0}, T<T_{0}$. Then from (1) we have that

$$
\begin{gathered}
\sum_{1<i / 2+j \leq 2} \tau^{1-i / 2-j} T^{-i / 2-j}\left\|E_{i / 2} D_{1}^{j} u\right\|_{T}^{(\tau)} \leq \tau^{-3 / 2} T^{-1}\left(\|\| D_{1} u\|\| T_{, 2}^{(\tau)}+\|\| E_{1} u\|\|_{T, 2}^{(\tau)}\right) \\
\leq C_{0} \tau^{-1 / 2} T^{-1}\left(\left\|L_{j}(x, D) D_{1} u\right\|_{T}^{(\tau)}\right. \\
\left.+\left\|L_{j}(x, D) E_{1} u\right\|_{T}^{(\tau)}\right) \\
\sum_{i / 2+j \leq 1} \tau^{1-i / 2-j} T^{-i / 2-j}\left\|E_{i / 2} D_{1}^{j} u\right\|_{T}^{(\tau)}=\tau^{-1 / 2}\|\| u\|\|_{T, 2}^{(\tau)} \leq C_{0}^{2}\left\|\left(L_{i} \circ L_{j}\right)(x, D) u\right\|_{T}^{(\tau)}
\end{gathered}
$$

From the proof of Lemma 5.2 we have with a notation in it that

$$
\left(L_{j} \circ\left\langle\xi^{\prime}\right\rangle\right)(x, \xi)=\left(\left\langle\xi^{\prime}\right\rangle \circ L_{j}\right)(x, \xi)+\int_{0}^{1} \sum_{|\alpha|=1} h_{\theta}\left[\partial_{\xi^{\prime}}^{\alpha}\left\langle\xi^{\prime}\right\rangle, D_{x^{\prime}}^{\alpha}\left(a_{j}+b_{j}\right)\right]\left(x, \xi^{\prime}\right) d \theta .
$$

Thus $\left[L_{j}(x, D), E_{1}\right] \in O p S_{\phi, \varphi}^{1,-1}$ from the assumption. We also have $\left[L_{j}(x, D), D_{1}\right]$ $\in O p S_{\mathscr{\phi}, \varphi}^{1,-1}$ from the assumption. Thus from (6.8) and (3.6) we have that

$$
\begin{gathered}
\sum_{1<i / 2+j \leq 2} \tau^{1-i / 2-j} T^{-i / 2-j}\left\|E_{i / 2} D_{1}^{j} u\right\|_{T}^{(\tau)} \leq C_{0} \tau^{-1 / 2} T^{-1}\left(\left\|D_{1} L_{j}(x, D) u\right\|_{T}^{(\tau)}\right. \\
\left.\left.+\left\|E_{1} L_{j}(x, D) u\right\|_{T}^{(\tau)}\right)+C_{0} C_{1} \tau^{-1 / 2} T^{-1}\left\|E_{1} u\right\|_{T}^{(\tau)}\right) \\
\left.\leq C_{0}^{2}\left(1+C_{0} C_{1} \tau^{-1 / 2}\right)\left\|\left(L_{i} \circ L_{j}\right)(x, D) u\right\|_{T}^{(\tau)}\right)
\end{gathered}
$$

This inequality and (6.9) imply the first inequality in (2). Next, we show the second one. From the first inequality we have

$$
\begin{gathered}
\sum_{2<i / 2+j \leq 3} \tau^{3 / 2-i / 2-j} T^{-i / 2-j}\left\|E_{i / 2} D_{1}^{j} u\right\|_{T}^{(\tau)} \leq \tau^{-1} T^{-1}\left(\left\|\mid D_{1} u\right\|\left\|_{T, 4}^{(\tau)}+\right\| E_{1} u \|_{T, 4}^{(\tau)}\right) \\
\leq C_{2} \tau^{-1 / 2} T^{-1}\left(\left\|\left(L_{2} \circ L_{3}\right)(x, D) D_{1} u\right\|_{T}^{(\tau)}\right. \\
\left.+\left\|\left(L_{2} \circ L_{3}\right)(x, D) E_{1} u\right\|_{T}^{(\tau)}\right) \\
\sum_{i / 2+j \leq 2} \tau^{3 / 2-i / 2-j} T^{-i / 2-j}\left\|E_{i / 2} D_{1}^{j} u\right\|_{T}^{(\tau)}=\|\| u\|\|_{T, 4}^{(\tau)} \leq C_{3}\left\|\left(L_{1} \circ L_{2} \circ L_{3}\right)(x, D) u\right\|_{T}^{(\tau)}
\end{gathered}
$$

if $\tau T^{2}$ and $T^{-1}$ are large. We use identities that 


$$
\begin{aligned}
& {\left[\left(L_{2} \circ L_{3}\right)(x, D), E_{1}\right]=\left[L_{2}(x, D),\left[L_{3}(x, D), E_{1}\right]\right]+\sum_{\{i, j\}=\{2,3\}}\left[L_{i}(x, D), E_{1}\right] L_{i}(x, D),} \\
& {\left[\left(L_{2} \circ L_{3}\right)(x, D), D_{1}\right]=\left[L_{2}(x, D),\left[L_{3}(x, D), D_{1}\right]\right]+\sum_{\{i, j\}=\{2,3\}}\left[L_{i}(x, D), D_{1}\right] L_{j}(x, D) .}
\end{aligned}
$$

If $r_{j}=L_{j} \circ\left\langle\xi^{\prime}\right\rangle-\left\langle\xi^{\prime}\right\rangle \circ L_{j}, r_{j} \in S_{\phi^{\prime}, \varphi^{-1}}^{1}$ as showed above and $\left[L_{i}(x, D), r_{j}\left(x, D^{\prime}\right)\right]$ $\in O p S_{\phi, \varphi}^{1,-2}$ similarly from the proof of Lemma 5.2. We also have $\left[L_{i}(x, D)\right.$, $\left.\left[L_{j}(x, D), D_{1}\right]\right] \in O p S_{\phi, \varphi}^{1,-2}$, since $\left[L_{j}(x, D), D_{1}\right] \in O p S_{\phi_{,},{ }^{-1}}^{1}$. Using these facts for above identities and noting (3.6) and Lemma 3.1 we get from (6.10) that

$$
\begin{aligned}
\sum_{2<i / 2+j \leq 3} \tau^{3 / 2-i / 2-j} T^{-i / 2-j} & \left\|E_{i / 2} D_{1}^{j} u\right\|_{T}^{(\tau)} \leq C_{4} \tau^{-1 / 2} T^{-1}\left(\left\|D_{1}\left(L_{2} \circ L_{3}\right)(x, D) u\right\|_{T}^{(\tau)}\right. \\
& \left.+\left\|E_{1}\left(L_{2} \circ L_{3}\right)(x, D) u\right\|_{T}^{(\tau)}\right) \\
& +C_{5} \tau^{-1 / 2} T^{-1}\left(\left\|E_{3 / 2} u\right\|_{T}^{(\tau)}+\sum_{j=2,3}\left\|E_{1} L_{j}(x, D) u\right\|_{T}^{(\tau)}\right) \\
\leq & C_{6} \sum_{\{i, j\}=\{2,3\}}\left\|\left(L_{1} \circ L_{i} \circ L_{j}\right)(x, D) u\right\|_{T}^{(\tau)}
\end{aligned}
$$

if $\tau T^{2}$ and $T^{-1}$ are large. This inequality and (6.11) proves the desired inequality. This completes the proof.

\section{§7. Proofs of Lemmas in $\S 3$}

Proof of Lemma 3.1. For some $C>0,\left\|a\left(x, D^{\prime}\right) u\left(x_{1},{ }^{\circ}\right)\right\| \leq C\left\|E_{m} u\left(x_{1},{ }^{\circ}\right)\right\|$ for any $x_{1} \in \mathbb{R}$, since $\left\{a\left(x_{1},{ }^{\circ}\right)\right\}_{x_{1} \in \mathbb{R}}$ is a bounded set in $S_{1 / 2,1 / 2}^{m}\left(\mathbb{R}^{n} \times \mathbb{R}^{n-1}\right)$. Multiplying this inequality by $e^{\tau\left(x_{1}-T\right)^{2}}$ and integrating on $[0, T]$ in $x_{1}$ we get the desired inequality.

Q.E.D.

Proof of Lemma 3.2. The proof needs three lemmas.

Lemma 7.1. (1) Let $L=\xi_{1}-\lambda-\mu$ with $\lambda \in S_{1,0}^{1}\left(\mathbb{R}^{n} \times \mathbb{R}^{n-1}\right)$ and $\mu \in S_{\mathscr{\phi}, \varphi}^{1,0}$. Let $a \in S_{\Phi, \varphi}^{M, m}\left(\right.$ resp. $\left.S_{1,0}^{m}\left(\mathbb{R}^{n} \times \mathbb{R}^{n-1}\right)\right)$. Then we have that

$$
a \circ L-a L, L \circ a-a L \in S_{\Phi, \varphi}^{M, m-1}\left(\operatorname{resp} . S_{\Phi, \varphi}^{m,-m}\right) .
$$

(2) Let $L_{i}(i=1,2)$ be as $L$ in $(1)$ with $\lambda_{i}$, $\mu_{i}$ respectively for $\lambda, \mu$. Let $a \in S_{\Phi, \varphi}^{M, m}$ (resp. $S_{1,0}^{m}\left(\boldsymbol{R}^{n} \times \boldsymbol{R}^{n-1}\right)$ ). Then there exist $a_{1}, a_{2} \in S_{\Phi, \varphi}^{M-1}$ (resp. $\left.S_{\phi, \varphi}^{m,-m}\right), a_{0} \in$ $S_{\Phi, \varphi}^{M, m-2}\left(\right.$ resp. $\left.S_{\phi, \varphi}^{m,-m}\right)$ such that

$$
a \circ\left(L_{1} L_{2}\right)-a L_{1} L_{2}=\sum_{i=1}^{2} a_{i} \circ L_{i}+a_{0}
$$

Proof. (1) We only prove that $a \circ L-a L \in S_{\Phi, \varphi}^{M, m-1}$ if $a \in S_{\Phi, \varphi}^{M, m}$. The others are proved similarly by using the fact that $S_{1,0}^{m} \subseteq S_{\Phi, \varphi}^{n,-m}$ in the case that $a \in S_{\Phi, \varphi}^{M, m}$. From Lemma 5.2 we have with a notation in its proof that 


$$
(a \circ(\lambda+\mu)-a(\lambda+\mu))\left(x, \xi^{\prime}\right)=\int_{0}^{1} \sum_{|\alpha|=1} h_{\theta}\left[\partial_{\xi^{\prime}}^{\alpha} a, D_{x^{\prime}}^{\alpha}(\lambda+\mu)\right]\left(x, \xi^{\prime}\right) d \theta .
$$

Since $D_{x^{\prime}}^{\alpha}(\lambda+\mu) \in S_{\phi^{\prime}, \varphi^{1}}^{1,-1}$ for $|\alpha|=1$, the right hand side of the above equality belongs to $S_{\Phi, \varphi}^{M, m-1}$ from the proof of Lemma 5.2.

(2) We only prove the case that $a \in S_{\varphi, \varphi}^{M, m-1}$. The other case is proved similarly. Using Lemma 5.2 for $a \circ\left(L_{1} L_{2}\right)$ freezing the variable $\xi_{1}$, we have

$$
\begin{gathered}
a \circ\left(L_{1} L_{2}\right)(x, \xi)=\left(a L_{1} L_{2}\right)(x, \xi)+\int_{0}^{1} r_{\theta}(x, \xi) d \theta, \\
r_{\theta}(x, \xi)=(2 \pi)^{-(n-1)} O S-\iint e^{-i y^{\prime} \cdot \eta^{\prime}} \sum_{j=2}^{n} \partial_{\xi_{j}} a\left(x, \xi^{\prime}+\theta \eta^{\prime}\right) \\
\times \sum_{\{k, l\}=\{1,2\}}\left(D_{x_{j}} L_{k} \cdot L_{l}\right)\left(\left(x_{1}, y^{\prime}+x^{\prime}\right), \xi\right) d y^{\prime} d \eta^{\prime} .
\end{gathered}
$$

Applying Taylor's formula for $L_{l}\left(\left(x_{1}, y^{\prime}+x^{\prime}\right), \xi\right)$ in $y^{\prime}$ and integrating by parts we see that

$$
r_{\theta}(x, \xi)=r_{1 \theta}(x, \xi)+r_{2 \theta}\left(x, \xi^{\prime}\right)
$$

where

$$
\begin{gathered}
r_{1 \theta}(x, \xi) \\
=-\sum_{\{k, l\}=\{1,2\}}\left((2 \pi)^{-(n-1)} O S-\iint e^{-i y^{\prime} \cdot \eta^{\prime}} \sum_{j=2}^{n} \partial_{\xi_{j}} a\left(x, \xi^{\prime}+\theta \eta^{\prime}\right)\right. \\
\left.\times D_{x j}\left(\lambda_{k}+\mu_{k}\right)\left(\left(x_{1}, y^{\prime}+x^{\prime}\right), \xi^{\prime}\right) d y^{\prime} d \eta^{\prime}\right) L_{l}(x, \xi), \\
r_{2 \theta}\left(x, \xi^{\prime}\right) \\
=-\sum_{\{k, l\}=\{1,2\}}(2 \pi)^{-(n-1)} O S-\iint e^{-i y^{\prime} \cdot \eta^{\prime}} \theta \sum_{s, j=2}^{n} \partial_{\xi_{s}} \partial_{\xi_{j}} a\left(x, \xi^{\prime}+\theta \eta^{\prime}\right) \\
\times D_{x_{j}}\left(\lambda_{k}+\mu_{k}\right)\left(\left(x_{1}, y^{\prime}+\eta^{\prime}\right), \xi^{\prime}\right)\left(\int_{0}^{1} \partial_{x_{s}}\left(\lambda_{l}+\mu_{l}\right)\left(\left(x_{1}, x^{\prime}+t y^{\prime}\right), \xi^{\prime}\right) d t\right) d y^{\prime} d \eta^{\prime} .
\end{gathered}
$$

Thus using a notatoin in the proof of Lemma 5.2 we have

$$
\int_{0}^{1} r_{1 \theta}(x, \xi) d \theta=-\sum_{\{k, l\}=\{1,2\}} \sum_{j=2}^{n} \int_{0}^{1} h_{\theta}\left[\partial_{\xi_{j}} a, D_{x j}\left(\lambda_{k}+\mu_{k}\right)\right]\left(x, \xi^{\prime}\right) d \theta L_{l}(x, \xi) .
$$

On the right of this equality, the coefficient of $L_{l}$ belongs to $S_{\phi, \varphi}^{M, m-1}$. Thus from (1), $\int_{0}^{1} r_{\theta}(x, \xi) d \theta$ takes the form of the right of (7.1). Therefore, to complete the proof of (2), it suffices to show that $\left\{r_{2 \theta}\right\}_{\theta \in[0,1]}$ is a bounded subset of $S_{\emptyset, \varphi}^{M, m-2}$. This follows from the following Lemma 7.2 and Fact 7.1. Q.E.D.

Lemma 7.2. Let $\left(M_{1}, \cdots, M_{N}, m_{1}, \cdots, m_{N}\right)$ be a permutation of $2 N$ real numbers, and let $a_{j}\left(x, y^{\prime}, \xi^{\prime}, \eta^{\prime}\right) \in C^{\infty}\left(\boldsymbol{R}_{x}^{n} \times \boldsymbol{R}_{\left(y^{\prime}, \xi^{\prime}, \eta^{\prime}\right)}^{3(n-1)}\right)(j=1, \cdots, N)$ satisfying the 
estimates that

$$
\left|\partial_{x}^{\alpha} \partial_{y^{\prime}}^{\beta} \partial_{\xi^{\prime}}^{\nu} \partial_{\eta^{\prime}}^{\mu} a_{j}\left(x, y^{\prime}, \xi^{\prime}, \eta^{\prime}\right)\right| \leq C_{\alpha \beta \nu \mu} \sup _{Q \in \mathbb{K}} \Phi_{x_{1}}^{M_{j}-|\nu|-\mid \mu^{\prime}}(Q) \varphi_{x_{1}}^{n_{j}-|\alpha|-|\beta|}(Q)
$$

for all multi-indices where we use a notation $K$ in (5.10). Then if we set $a=$ $I\left[a_{1}, \cdots, a_{N}\right]$ with

$$
\begin{aligned}
& I\left[a_{1}, \cdots, a_{N}\right]\left(x, \xi^{\prime}\right) \\
& \quad=(2 \pi)^{-(n-1)} O S-\iint e^{-i y^{\prime} \cdot \eta^{\prime}} \prod_{j=1}^{\Delta J} a_{j}\left(x, y^{\prime}+x^{\prime}, \xi^{\prime}, \eta^{\prime}+\xi^{\prime}\right) d y^{\prime} d \eta^{\prime},
\end{aligned}
$$

we have $a \in S_{\Phi, \varphi}^{M, m}$ and $|a|_{l} \leq C_{l} \prod_{j=1}^{N}\left|a_{j}\right|_{L_{0}+l}$ where $M=\sum_{j=1}^{n} M_{j}, m=\sum_{j=1}^{N} m_{j}$, $\left|a_{j}\right|_{l}=\max _{\{(\alpha, \beta \nu, \mu) \mid \leq l}\left\{\right.$ infimum of $C_{\alpha \beta \nu^{\mu}}$ in $\left.(7.2)\right\}$, and the constants $C_{0}, L_{0}$ are depending only on $l, \Phi, \varphi$, and a permutation given above.

Fact 7.1. Let $a \in S_{\Phi, \varphi}^{M, m}$. Then

$$
\begin{aligned}
& \sup _{\substack{0 \leq t \leq 1 \\
0 \leq s \leq 1}}\left|\partial_{x}^{\alpha} \partial_{y^{\prime}}^{\beta} \partial_{\xi^{\prime}}^{\nu} \partial_{\eta^{\prime}}^{\mu}\left[a\left(x+t\left(0, y^{\prime}-x^{\prime}\right), \xi^{\prime}+s\left(\eta^{\prime}-\xi^{\prime}\right)\right)\right]\right| \\
& \leq|a|_{l}\left(\Phi_{x_{1}}^{M-|\nu|-|\mu|} \varphi_{x_{1}}^{m-|\alpha|-|\beta|}\right)\left((1-t) x^{\prime}+t y^{\prime},(1-s) \xi^{\prime}+s \eta^{\prime}\right) \\
& \text { if }|\alpha|+|\beta|+|\nu|+|\mu| \leq l .
\end{aligned}
$$

Fact 7.1 is obvious. We shall prove Lemma 7.2.

Proof of Lemma 7.2. Set $b=\prod_{j=1}^{N} a_{j}$. Then we have the estimate

$$
\begin{aligned}
& \left|\partial_{y^{\prime}}^{\alpha}, \partial_{\eta^{\prime}}^{\beta} b\left(x, y^{\prime}, \xi^{\prime}, \eta^{\prime}\right)\right| \\
\leq & N^{|\alpha|+|\beta|} \prod_{j=1}^{N}\left|a_{j}\right|_{|\alpha|+|\beta|} \sum_{\substack{\Sigma_{\alpha j=\alpha} \alpha_{j}=\beta \\
\Sigma}} \prod_{j=1}^{N}\left(\sup _{Q \in \mathbb{K}} \Phi_{\beta_{1}^{j}=\beta}^{M_{j}-\left|\beta^{j}\right|}(Q) \sup _{Q \in \mathbb{K}} \varphi_{x_{1}}^{m_{j}-\left|\alpha^{j}\right|}(Q)\right) .
\end{aligned}
$$

Thus from Lemma $5.2\left|a\left(x, \xi^{\prime}\right)\right| \leq C_{0} \Pi_{j=1}^{N}\left|a_{j}\right|_{L_{0}}\left(\Phi^{M} \varphi^{m}\right)\left(x, \xi^{\prime}\right)$. Differentiating under integral sign we see that

$$
\begin{aligned}
& \partial_{x}^{\alpha} \partial_{\xi^{\prime}}^{\beta} a\left(x, \xi^{\prime}\right) \\
& =\sum_{\Sigma \nu^{j}+\left(0, \tilde{\nu}^{j}\right)=\alpha} \frac{\alpha !}{\nu^{1} ! \cdots \nu^{N} ! \tilde{v}^{1} ! \cdots \tilde{\nu}^{N} !} \frac{\beta !}{\mu^{1} ! \cdots \mu^{N} ! \tilde{\mu}^{1} ! \cdots \tilde{\mu}^{N} !} \\
& \Sigma \mu^{j}+\tilde{\mu} j=\beta \\
& \times I\left[\partial_{x}^{\nu^{1}} \partial_{y^{\prime}}^{\tilde{\nu}} \partial_{\xi^{\prime}}^{\mu 1} \partial_{\eta^{\prime}}^{\tilde{\mu}^{1}} a_{1}, \cdots, \partial_{x}^{\nu N} \partial_{y^{\prime}}^{\tilde{\nu} N} \partial_{\xi^{\prime}}^{\mu N} \partial_{\eta^{\prime}}^{\tilde{\mu} N} a_{N}\right] .
\end{aligned}
$$

Thus from above estimate we have

$$
\left|\partial_{x}^{\alpha} \partial_{\xi^{\prime}}^{\beta} a\left(x, \xi^{\prime}\right)\right| \leq C_{0}(2 N)^{|\alpha|+|\beta|} \prod_{j=1}^{N}\left|a_{j}\right|_{L_{0}+|\alpha|+|\beta|}\left(\Phi^{M-|\beta|} \varphi^{m-|\alpha|}\right)\left(x, \xi^{\prime}\right) .
$$

Q.E.D. 
Lemma 3.2 is an immediate consequence of the second part of the next lemma and Proposition 3.2.

Lemma 7.3. Let $L_{i}=\xi_{1}-\lambda_{i}-\mu_{i}(i=1,2,3)$ with $\lambda_{i} \in S_{1,0}^{1}\left(\boldsymbol{R}^{n} \times \boldsymbol{R}^{n-1}\right), \mu_{i} \in$ $S_{\phi, \varphi}^{1,0}$. Then we have the followings.

(1) $L_{1} \circ L_{2}-L_{1} L_{2} \in S_{\phi, \varphi}^{1,-1}$.

(2) For some $a_{j} \in S_{\mathscr{\phi}, \varphi}^{1,-1}(j=1,2,3)$ and $a_{0} \in S_{\mathscr{\phi}, \varphi}^{1,-2}$ we have that

$$
L_{1} \circ L_{2} \circ L_{3}-L_{1} L_{2} L_{3}=\sum_{j=1}^{3} a_{j} \circ L_{j}+a_{0} .
$$

Proof. (1) we have

$$
\begin{aligned}
& L_{1} \circ L_{2}-L_{1} L_{2} \\
& \quad=-D_{x_{1}}\left(\lambda_{2}+\mu_{2}\right)+\left(\lambda_{1} \circ L_{2}-\lambda L_{2}\right)+\left(\mu_{1} \circ L_{2}-\mu_{1} L_{2}\right) .
\end{aligned}
$$

Each of terms on the right hand side belongs to $S_{\mathscr{\phi}, \varphi}^{1,-1}$ from the assumption and Lemma 7.1.

(2) In view of (1) and Lemma 7.1-(1) $L_{1} \circ L_{2} \circ L_{3}-L_{1} \circ\left(L_{2} L_{3}\right)$ takes the form of the right of (7.3). Thus it suffices to show that $L_{1} \circ\left(L_{2} L_{3}\right)-L_{1} L_{2} L_{3}$ does also. We have

$$
\begin{aligned}
L_{1} \circ\left(L_{2} L_{3}\right)-L_{1} L_{2} L_{2}= & \sum_{\{k, l\}=\{2,3\}} D_{x_{1}} L_{k} \cdot L_{l} \\
& -\left\{\lambda_{1} \circ\left(L_{2} L_{3}\right)-\lambda_{1} L_{2} L_{3}\right\}-\left\{\mu_{1} \circ\left(L_{2} L_{3}\right)-\mu_{1} L_{2} L_{3}\right\} .
\end{aligned}
$$

Each term on the right hand side takes the form of the right of (7.3) from Lemma 7.1.

Q.E.D.

This completes the proof of Lemma 3.2.

Proof of Lemma 3.3. Take $a=\chi, q=p$ in Lemma 5.6 with $N=3$ and in Lemma 5.7. with $L=1$ and $N=3$. Then Lemma 3.3 follows from Corollary $5.1,5.2$.

Q.E.D.

Proof of Lemma 3.4. This follows from Lebiniz rule.

Q.E.D.

Proof of Lemma 3.5. From Proposition 3.2 and Lemma 3.2.

$$
B(u) \leq C_{1}\|(p-q)(x, D) u\|_{T}^{(\tau)}
$$

if $\tau T^{2}, \frac{1}{T}$ are large. Since $g \circ \chi \in S_{\phi, \varphi}^{0,-3} \subset S_{1 / 2,1 / 2}^{3 / 2}$, we have

$$
\left\|g \circ \chi\left(x, D^{\prime}\right) u\right\|_{T}^{(\tau)} \leq C_{2}\left\|E_{3 / 2} u\right\|_{T}^{(\tau)}
$$


From these two inequalities we see that for large $\tau T^{2}, \frac{1}{T}$ $\left\|B\left(\chi\left(x, D^{\prime}\right) u\right)\right\|_{T}^{(\tau)}+\left\|g \circ \chi\left(x, D^{\prime}\right) u\right\|_{T}^{(\tau)} \leq C_{1}\left\|p \circ \chi\left(x, D^{\prime}\right) u\right\|_{T}^{(\tau)}+C_{2}\left(1+C_{1}\right)\left\|E_{3 / 2} u\right\|_{T}^{(\tau)}$.

Applying Lemma 3.3 to the first term on the right we get the desired inequality.

Q.E.D.

Proof of Lemma 3.6. Taking $\Phi\left(x, \xi^{\prime}\right)=\left\langle\xi^{\prime}\right\rangle, \varphi\left(x, \xi^{\prime}\right)=1$ in Lemma 7.1, 7.3-(1), we have that if $|\alpha|+|\beta|=1$ (resp. 2)

$$
\begin{aligned}
\partial_{\xi}^{\alpha} \partial_{x}^{\beta}(p-g)=\sum_{i \leq j} a_{i j} \circ L_{0 \dot{\xi}} \circ L_{0 j} \\
+\sum_{i=1}^{2} a_{i} \circ L_{0 i}+a_{0}
\end{aligned}
$$

with $a_{i j} \in S_{1,0}^{1-|\alpha|}, a_{i} \in S_{1,0}^{1-|\alpha|}\left(\right.$ resp. $\left.S_{1,0}^{2-|\alpha|}\right)$ for $i \neq 0, a_{0} \in S_{0,1}^{2-|\alpha|}$

From this we have that when $|\alpha|+|\beta|=1$ or 2 ,

$$
\begin{aligned}
& \left\langle\xi^{\prime}\right\rangle^{(|\alpha|-|\beta|) / 2} \circ \partial_{\xi}^{\alpha} \partial_{x}^{\beta}(p-g)=\sum_{i \leq j} a_{i j} \circ L_{0 i} \circ L_{0 j} \\
& +\sum_{i=1}^{2} a_{i} \circ L_{0 i}+a_{0}
\end{aligned}
$$

with $a_{i j} \in S_{1,0}^{1 / 2}, a_{i} \in S_{1,0}^{1}(i \neq 0), a_{0} \in S_{1,0}^{3 / 2}$.

Thus Proposition 3.2 implies that if $\tau T^{2}$ and $\frac{1}{T}$ are large

$$
\left.T^{-1 / 2} \sum_{|\alpha|+|\beta|=1,2}\left\|E_{(|\alpha|-|\beta|) / 2}(p-g)_{(\beta)}^{(\alpha)}(x, D) u\right\|_{T}^{(\tau)} \leq C_{1} \|(p-g)\right)(x, D) u \|_{T}^{(\tau)} .
$$

Thus using $\left\langle\xi^{\prime}\right\rangle^{(|\alpha|-|\beta|) / 2} \circ g_{(\beta)}^{(\alpha)} \chi \in S_{\Phi^{\prime}, \varphi^{-3}}^{0,-3}$ and Lemma 3.2 we get the desired inequality.

Q.E.D.

Proof of Lemma 3.7. As in the proof of Lemma 3.3 we take $q=p, a=\chi$, $N=3$ in Lemma 5.6 and apply Corollary 5.1. Then we have with some $b_{i} \in$ $S_{1 / 2,1 / 2}^{i-1 / 2}$ and constants $C_{\alpha}$

$$
\chi \circ p=\chi p+\sum_{|\alpha|=1,2} C_{\alpha} \partial_{\xi^{\prime}}^{\alpha} a \circ \partial_{x^{\prime}}^{\alpha} p+\sum_{i=0}^{2} b_{i} \xi_{1}^{2-i}
$$

Thus Lemma 3.7 follows from the following lemma and Lemma 7.1, 7.3(1).

Q.E.D.

Lemma 7.4. Let $L_{i}(i=1,2,3)$ be as in Lemma 7.3 and $a \in S_{\Phi, \varphi}^{M, m}$. Then

$$
\left(L_{1} L_{2} L_{3}\right) \circ a=a L_{1} L_{2} L_{3}+\sum_{i \neq j} a_{i j} L_{i} L_{j}+\sum_{i=1}^{3} a_{i} L_{i}+a_{0}
$$


with some $a_{i j} \in S_{\Phi, \varphi}^{M, m-1}, a_{i} \in S_{\Phi, \varphi}^{M, m-2}(i \neq 0), a_{0} \in S_{\Phi, \varphi}^{M, m-3}$.

Proof. We have

$$
\begin{aligned}
& \left(L_{1} L_{2} L_{3}\right) \circ a(x, \xi) \\
= & \left.(2 \pi)^{-(n-1)} O S-\iint e^{-i y^{\prime} \cdot \eta^{\prime}}\left(L_{1} L_{2} L_{3}\right)\left(x, \xi+\left(0, \eta^{\prime}\right)\right) a\left(x+0, y^{\prime}\right), \xi^{\prime}\right) d y^{\prime} d \eta^{\prime} \\
+ & \sum_{l>j}(2 \pi)^{-(n-1)} O S-\iint e^{-i y^{\prime} \cdot \eta^{\prime}}\left(L_{l} L_{j}\right)\left(x, \xi+\left(0, \eta^{\prime}\right)\right) D_{x_{1}} a\left(x+\left(0, y^{\prime}\right), \xi^{\prime}\right) d y^{\prime} d \eta^{\prime} \\
+ & \sum_{l=1}^{3}(2 \pi)^{-(n-1)} O S-\iint e^{-i y^{\prime} \cdot \eta^{\prime}} L_{l}\left(x, \xi+\left(0, \eta^{\prime}\right)\right) D_{x_{1}}^{2} a\left(x+\left(0, y^{\prime}\right), \xi^{\prime}\right) d y^{\prime} d \eta^{\prime} \\
+ & (2 \pi)^{-(n-1)} \iint e^{-i y^{\prime} \cdot \eta^{\prime}} D_{x_{1}}^{3} a\left(x+\left(0, y^{\prime}\right), \xi^{\prime}\right) d y^{\prime} d \eta^{\prime} \\
= & I+\cdots+I V .
\end{aligned}
$$

Since $(2 \pi)^{-(n-1)}$ OS $-\iint e^{-i y^{\prime} \cdot \eta^{\prime}} a\left(x+\left(0, y^{\prime}\right), \xi^{\prime}\right) d y^{\prime} d \eta^{\prime}=a\left(x, \xi^{\prime}\right)$ by Fourier inversion formula and a limiting argument, in the term I we see using Taylor's formula for $L_{l}\left(x, \xi+\left(0, \eta^{\prime}\right)\right)$ in $\eta^{\prime}$ and the integration by parts

$$
\begin{aligned}
I= & \prod_{l=1}^{3} L_{l}(x, \xi) a\left(x, \xi^{\prime}\right) \\
& -\sum_{l=1}^{3}(2 \pi)^{-(n-1)} O S-\iint e^{-i y^{\prime} \cdot \eta^{\prime}} \sum_{\left|\alpha_{\mid}\right|=1} \int_{0}^{1} \partial_{\xi^{\prime}}^{\alpha}\left(\lambda_{l}+\mu_{l}\right)\left(x, \xi^{\prime}+\theta \eta^{\prime}\right) \\
& \times D_{x^{\prime}}^{\alpha} a\left(x+\left(0, y^{\prime}\right), \xi^{\prime}\right) d y^{\prime} d \eta^{\prime} \prod_{j \neq l} L_{j}(x, \xi) \\
& -\sum_{j_{1}, j_{2}, j_{3}: d i s t i n c t}(2 \pi)^{-(n-1)} O S-\iint e^{i y^{\prime} \cdot \eta^{\prime}} \\
& \left.\times \sum_{\left|\alpha^{1}\right|,\left|\alpha^{2}\right|=1}\left\{\prod_{k=1}^{2} \int_{0}^{1} \partial_{\xi^{\prime}}^{\alpha k}\left(\lambda_{k}+\mu_{k}\right)\left(x, \xi^{\prime}\right)+\theta \eta^{\prime}\right) d \theta\right\} D_{x^{\prime}}^{\alpha^{1}+\alpha^{\mu}} a\left(x+\left(0, y^{\prime}\right), \xi^{\prime}\right) d y^{\prime} d \eta^{\prime} \\
& \times L_{j_{3}}(x, \xi) \\
& -(2 \pi)^{-(n-1)} O S-\iint e^{-i y^{\prime} \cdot \eta} \sum_{\left|\alpha^{1}\right|,\left|\alpha^{2}\right|,\left|\alpha^{3}\right|=1}\left\{\prod_{k=1}^{3} \int_{0}^{1} \partial_{\xi^{\prime}}^{\alpha k}\left(\lambda_{k}+\mu_{k}\right)\left(x, \xi^{\prime}+\theta \eta^{\prime}\right) d \theta\right\} \\
& \times D_{x^{\prime}}^{\alpha^{1}+\alpha^{2}+\alpha^{3}} a\left(x+\left(0, y^{\prime}\right), \xi^{\prime}\right) d y^{\prime} d \eta^{\prime} .
\end{aligned}
$$

Similarly we have

$$
\begin{aligned}
I I & =\sum_{l>j} D_{x_{1}} a\left(x, \xi^{\prime}\right)\left(L_{l} L_{j}\right)(x, \xi) \\
& -\sum_{l \neq j}(2 \pi)^{-(n-1)} O S-\iint e^{-i y^{\prime} \cdot \eta^{\prime}} \sum_{|\alpha|=1} \int_{0}^{1} \partial_{\xi^{\prime}}^{\alpha}\left(\lambda_{l}+\mu_{l}\right)\left(x, \xi^{\prime}+\theta \eta^{\prime}\right) d \theta \\
& \times D_{x_{1}} D_{x^{\prime}}^{\alpha} a\left(x+\left(0, y^{\prime}\right), \xi^{\prime}\right) d y^{\prime} d \eta^{\prime} \cdot L_{j}(x, \xi)
\end{aligned}
$$




$$
\begin{aligned}
& \quad+\sum_{j_{1}>j_{2}}(2 \pi)^{-(n-1)} O S-\iint e^{-i y^{\prime} \cdot \eta^{\prime}} \sum_{\left|\alpha^{1}\right|,\left|\alpha^{2}\right|=1}\left\{\prod_{k=1}^{2} \int_{0}^{1} \partial_{\xi^{\prime}}^{\alpha k}\left(\lambda_{k}+\mu_{k}\right)\left(x, \xi^{\prime}+\theta \eta^{\prime}\right) d \theta\right\} \\
& \quad \times D_{x_{1}} D_{x^{\prime}}^{\alpha^{1}+\alpha^{2}} a\left(x+\left(0, y^{\prime}\right), \xi^{\prime}\right) d y^{\prime} d \eta^{\prime}, \\
& I I I=\sum_{l=1}^{3} D_{x_{1}}^{3} a\left(x, \xi^{\prime}\right) L_{l}(x, \xi) \\
& \quad-\sum_{l=1}^{3}(2 \pi)^{-(n-1)} O S-\iint e^{-i y^{\prime} \cdot \eta^{\prime}} \sum_{|\alpha|=1} \int_{0}^{1} \partial_{\xi^{\prime}}^{\alpha}\left(\lambda_{l}+\mu_{l}\right)\left(x, \xi^{\prime}+\theta \eta^{\prime}\right) d \theta \\
& \quad \times D_{x_{1}}^{2} D_{x^{\prime}}^{\alpha} a\left(x+\left(0, y^{\prime}\right), \xi^{\prime}\right) d y^{\prime} d \eta^{\prime}, \\
& I V=D_{x_{1}}^{3} a\left(x, \xi^{\prime}\right) .
\end{aligned}
$$

As in the proof of Lemma 7.1-(2) applying Lemma 7.2 to oscillaroty integrals in $I \sim$ III we see that $I+\cdots+I V$ is the form of the right of the equality in Lemma 7.4 .

Q.E.D.

Proof of Lemma 3.8. This follows easily from the following lemma and Lemma 7.3-(1).

Q.E.D.

Lemma 7.5. Let $a \in S_{\phi, \varphi}^{M, m}$, and let $L_{\dot{8}}(i=1,2)$ be as in Lemma 7.3. Set $L_{0 \dot{s}}=\xi_{1}-\lambda_{i}$. Then

$$
\left(L_{1} L_{2}\right) \circ a=\left(L_{01} L_{02}\right) \circ a+\sum_{l=1}^{2} b_{i} \circ L_{0 i}+b_{0}
$$

with $b_{\dot{i}} \in S_{\Phi, \varphi}^{M+1, m}(i=1,2)$ and $b_{0} \in S_{\Phi, \varphi}^{M+2, m}$, and

$$
L_{1} \circ a=L_{01} \circ a+a_{1}
$$

with $a_{1} \in S_{\Phi . \varphi}^{M+1, m}$.

Proof. We have

$$
L_{1} L_{2}=L_{01} L_{02}-\mu_{1} L_{02}-\mu_{2} L_{01}+\mu_{1} \mu_{2} .
$$

Thus from Lemma 7.1-(1) we have with some $b \in S_{\phi, \varphi}^{1,-1}$

$$
\left(L_{1} L_{2}\right) \circ a=\left(L_{01} L_{02}\right) \circ a-L_{02} \circ\left(\mu_{1} \circ a\right)-L_{01} \circ\left(\mu_{2} \circ a\right)+\left(\mu_{1} \mu_{2}+b\right) \circ a .
$$

Hence the last term on the right is in $S_{\Phi, \varphi}^{M+2, m}+S_{\Phi, \varphi}^{M+1, m-1} \subset S_{\Phi_{\bullet} \varphi}^{M+2, m}$. Thus applying Lemma 7.1-(1) to the middle two terms on the right we obtain the first statement. The second statement is trivial.

Q.E.D.

Proof of Lemma 3.9. We prove (1) first. We need a lemma. 
Lemma 7.6. Assume the notations in $\$ 3$. Assume (I) holds.

(1) For any distinct $1 \leq k, l \leq 3$

$$
L_{01} \psi_{0}=a_{1} L_{k}+a_{2} L_{l}
$$

with some $a_{i} \in S_{\Phi ; \varphi}^{0,0}(i=1,2)$ with suppa $a_{i} \subset \operatorname{supp} \psi_{0}$.

(2) For any distinct $1 \leq k, l \leq 3$ we have with some $a_{1}, a_{2}$ as in (1)

$$
\Phi \psi_{0}=a_{1} L_{k}+a_{2} L_{l} .
$$

Proof. (1) Set

$$
a_{1}=\psi_{0} \Lambda_{l} /\left(\Lambda_{l}-\Lambda_{k}\right), a_{2}=-\psi_{0} \Lambda_{k} /\left(\Lambda_{l}-\Lambda_{k}\right)
$$

with a trivial convention outside supp $\psi_{0}$. These have required properties.

(2) Set

$$
a_{1}=\psi_{0} \Phi /\left(\Lambda_{l}-\Lambda_{k}\right), a_{2}=-\psi_{0} \Phi /\left(\Lambda_{l}-\Lambda_{k}\right) .
$$

Then $a_{i} \in S_{\Phi, \varphi}^{0,0}(i=1,2)$ and satisfy the equality in (2).

Q.E.D.

If $k \neq l$, from Lemma 7.6-(1) and Lemma 7.1-(1) we have with some $a_{1}, a_{2} \in$ $S_{\phi, \varphi}^{0,0}, a_{0} \in S_{\varphi, \varphi}^{0,-1}$

$$
L_{01} \circ \psi_{0}=a_{1} \circ L_{k}+a_{2} \circ L_{l}+a_{0} .
$$

Since

$$
L_{01} \circ \psi_{1}=L_{01} \circ\left(1-\psi_{0}\right) \circ \psi_{1}+L_{01} \circ \psi_{0} \circ \psi_{1},
$$

the first statement in (1) follows. Next the proof of Lemma 5.2 shows that $c \circ \psi_{0}-c \psi_{0} \in S_{\Phi, \varphi}^{0,-1}$. From this and that $c \psi_{0} / \Phi \in S_{\Phi, \varphi}^{0,0}$ Lemma 5.2 shows that with $b_{0}=c \psi_{0} / \Phi$ and some $b_{1} \in S_{\phi, \varphi}^{0,-1}$

$$
c \circ \psi_{0} \circ \psi_{1}=b_{0} \circ\left(\Phi \psi_{0}\right) \circ \psi_{1}+b_{1} .
$$

Now, the second statement immediately follows from Lemmas 7.6-(2), 7.1-(1). This completes the proof of (1)

(2) can be proved similarly by using the following lemma

Q.E.D.

Lemma 7.7. Assume the notations in $§ 3$. Assume (II) holds.

(1) The statement of (1) in the previous lemma holds.

(2) If $k \neq 1$, we have with some $a_{i} \in S_{\Phi, \varphi}^{0,0}(i=1,2)$

$$
c \psi_{0}=a_{1} L_{1}+a_{2} L_{k}
$$

Proof. (1) If $k=2, l=3$, the same proof as that of Lemma 7.6-(1) works well. In another cases we set 


$$
a_{1}=-\psi_{0} \Lambda_{k} /\left(c+\Lambda_{1}-\Lambda_{k}\right), a_{2}=\psi_{0}\left(c+\Lambda_{1}\right) /\left(c+\Lambda_{1}-\Lambda_{k}\right)
$$

We have

$$
\left|c_{(a)}^{(\beta)}\left(x, \xi^{\prime}\right)\right| \leq C_{\alpha \beta}\left|c\left(x, \xi^{\prime}\right)\right|\left(\Phi^{-|\beta|} \varphi^{-|\alpha|}\right)\left(x, \xi^{\prime}\right) \text { for any } \alpha, \beta
$$

because

$$
\left|c_{(\alpha)}^{(\beta)}\left(x, \xi^{\prime}\right)\right| \leq C_{\alpha \beta}\left(\Phi^{1-|\beta|} \varphi^{-|\alpha|}\right)\left(x, \xi^{\prime}\right) \text { if }|\alpha|+|\beta|>0 .
$$

Using (7.4) we can easily check $a_{i} \in S_{\emptyset, \varphi}^{0,0}$ and the desired equality clearly holds.

(2) Set

$$
a_{1}=-\psi_{0} c /\left(c+\Lambda_{1}-\Lambda_{k}\right), \quad a_{2}=\varphi_{0} c /\left(c+\Lambda_{1}-\Lambda_{k}\right) .
$$

Then these have required properties.

Q.E.D.

Proof of Lemma 3.10. We need a lemma.

Lemma 7.8. Let $L_{i}(i=1,2,3)$ be as in Lemma 7.3 and set $q=\prod_{i=1}^{3} L_{i}$. Then we have for $\alpha, \beta$ with $1 \leq|\alpha|+|\beta| \leq 2$

$$
q_{(\beta)}^{(\alpha)}=\sum_{i>j} a_{i j} \prod_{j \in\{i, j\}} L_{l}
$$

with some $a_{i j} \in S_{\Phi, \varphi}^{1-|\alpha|,-|\beta|}(|\alpha|+|\beta|=1)$,

$$
q_{(\beta)}^{(\alpha)}=\sum_{i>j} a_{i j} \prod_{l \in\{i, j\}} L_{l}+\sum_{i=1}^{3} a_{i} L_{i}
$$

with some $a_{i j} \in S_{\Phi, \varphi}^{1-|\alpha|,-|\beta|}, a_{i} \in S_{\Phi, \varphi}^{2-|\alpha|,-|\beta|}(|\alpha|+|\beta|=2)$.

Proof. (i) Assume $|\alpha|+|\beta|=1$. If $\alpha_{1}=1,(7.5)$ is clear. If $\alpha_{1}=0$, we have

$$
q_{(\beta)}^{(\alpha)}=\sum_{\substack{i, j, k \\ i>d i s t i n c t}}-\left(\lambda_{k}+\mu_{k}\right)_{(\beta)}^{(\alpha)} \prod_{l \in\{i, j\}} L_{l}
$$

Here $\left(\lambda_{k}\right)_{(\beta)}^{(\alpha)} \in S_{1,0}^{1-|\alpha|} \subset S_{\Phi, \varphi}^{1-|\alpha|,-(1-|\alpha|)}=S_{\Phi, \varphi}^{1-|\alpha|,-|\beta|}$. Thus (7.5) holds.

(ii) Assume $|\alpha|+|\beta|=2$. If $\alpha_{1}=2,(7.6)$ is clear. If $\alpha_{1}=1$, we have

$$
q_{(\beta)}^{(\alpha)}=\sum_{i \neq j}-\left(\lambda_{i}+\mu_{i}\right)_{(\beta)}^{(\alpha \prime)} L_{j}
$$

Since $\left|\alpha^{\prime}\right|+|\beta|=1,\left(\lambda_{i}+\mu_{i}\right)_{(\beta)}^{\left(\alpha^{\prime}\right)} \in S_{\Phi, \varphi}^{1-\left|\alpha^{\prime}\right|-|\beta|}=S_{\Phi, \varphi}^{2-|\alpha|,-|\beta|}$. Thus (7.6) holds. If $\alpha_{1}=0$, we have

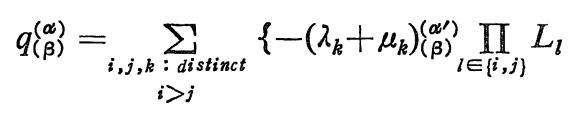




$$
+\sum_{\substack{\mu \leq \alpha^{\prime} \\
\nu \leq \beta \\
|\mu+\nu|=1}}\left(\begin{array}{c}
\alpha^{\prime} \\
\mu
\end{array}\right)\left(\begin{array}{l}
\beta \\
\nu
\end{array}\right)\left(\lambda_{i}+\mu_{i}\right)_{(\nu)}^{(\mu)}\left(\lambda_{j}+\mu_{j}\right)\left(\begin{array}{c}
\left(\alpha^{\prime}-\mu\right) \\
\beta
\end{array}\right.
$$

Here in the first term in the parenthis $\left(\lambda_{k}\right)_{(\beta)}^{\left(\alpha^{\prime}\right)} \in S_{\Phi, \varphi}^{1-|\alpha|,-|\beta|}$ because $-1+$ $|\alpha| \geq-|\beta|$, and in the second term $\left(\lambda_{i}+\mu_{i}\right)_{(\nu)}^{(\mu)} \in S_{\Phi, \varphi}^{1-|\mu|,-|\nu|},\left(\lambda_{j}+\mu_{j}\right)_{(j-\nu)}^{\left(\alpha^{\prime}-\mu\right)} \in$ $S_{\Phi, \varphi}^{1-\left|\alpha^{\prime}\right|+|\mu|,-|\beta|+|\nu|}$ for the same reason so that their product belongs to $S_{\Phi, \varphi}^{2-\left|\alpha^{\prime}\right|,-|\beta|}$. This shows (7.6).

Q.E.D.

Now we shall prove Lemma 3.10. From Lemma 5.7 we have with the notations in Lemma 5.7, 5.8, 5.9

$$
\begin{aligned}
p_{(\beta)}^{(\alpha)} \circ \chi & =\sum_{\substack{0 \leq j \leq 2 \\
|\gamma|+j \leq L-1}} \frac{1}{\gamma ! j !} \partial_{\xi}^{\alpha+(j, \gamma)} \partial_{x}^{(0, \beta)} p D_{x}^{(j, \gamma)} \chi \\
& +\sum_{\substack{0 \leq j \leq 2 \\
L \leq|\gamma|+j<N}} \sum_{|\mu|<N-|\gamma|-j} C_{\gamma \mu_{j}}(-i)^{|\mu|}\left[\partial_{\xi^{\prime}}^{\mu} D_{x}^{(j, \gamma)} \chi, p\right]_{\alpha+(j, \gamma)(0, \beta+\mu)}(1, x, \xi) \\
& +\sum_{\substack{0 \leq j \leq 2 \\
L \leq|\gamma|+j<N}} \frac{N-j}{\gamma ! j !} \int_{0}^{1}\left[p, D_{x}^{(j, \gamma)} \chi\right]_{\alpha+(j, \gamma)(0, \beta)}(\theta, x, \xi)(1-\theta)^{N-j-1} d \theta \\
& +\sum_{\substack{0 \leq j \leq 2 \\
L \leq|\gamma|+j<N}} \sum_{|\mu|=N-|\gamma|-j}(-i)^{|\mu|} \int_{0}^{1}\left[\partial_{\xi^{\prime}}^{\mu} D_{x}^{(j, \gamma)} \chi, p\right]_{\alpha+(j, \gamma)(0, \beta)}(\theta, x, \xi) \\
& \times \sum_{L \leq|\mu|-1} C_{\gamma \mu_{j} l}(1-\theta)^{|\mu|-1-l} d \theta \\
= & I+I I+I I I+I V .
\end{aligned}
$$

Assume $1 \leq|\alpha|+|\beta| \leq 2$ in the following. From Collorary 5.1 we have for any $j, r, \mu$

$$
\left[\partial_{\xi^{\prime}}^{\mu} D_{x}^{(j, \gamma)} \chi, p\right]_{\alpha+(j, \gamma)(0, \beta+\mu)}(\theta, x, \xi)= \begin{cases}\sum_{j=0}^{\min \left(2,3-\alpha_{1}-j\right)} b_{k \theta}^{\prime} \xi_{1}^{k} & \left(\alpha_{1}+j \leq 3\right) \\ 0 & \left(\alpha_{1}+j>3\right)\end{cases}
$$

with some $\left\{b_{k \theta}\right\}_{\theta \in[0,1]}$ being bounded in $S_{1 / 2,1 / 2}^{3-k-|\alpha|-(|(j, \gamma)|+|\mu|) / 2}\left(\boldsymbol{R}^{n} \times \boldsymbol{R}^{n-1}\right)$. From Corollary 5.2 we have for any $j, r$

$$
\left[p, D_{x}^{(j, \gamma)} \chi\right]_{(\alpha+(j, \gamma)(0, \beta)}(\theta, x, \xi)= \begin{cases}\sum_{k=0}^{\min \left(2,3-\alpha_{1}-j\right)} b_{k \theta}^{\prime} \xi_{1}^{k} & \left(\alpha_{1}+j \leq 3\right) \\ 0 & \left(\alpha_{1}+i>3\right)\end{cases}
$$

with some $\left\{b_{k \theta}^{\prime}\right\}_{\theta \in 0,1}$ being bounded in $S_{1 / 2,1 / 2}^{3-k-|\alpha|-|(j, \gamma)| / 2}\left(\boldsymbol{R}^{n} \times \boldsymbol{R}^{n-1}\right)$.

We devide our argument into two cases.

(i) Assume $|\alpha|+|\beta|=1$. Take $L=2, N=3$. Then from (7.7), (7.8) we have

$$
I I+I I I+I V=\sum_{k=0}^{\min \left(2,3-\alpha_{1}\right)} b_{k} \xi_{1}^{k} \quad \text { with some } \quad b_{k} \in S_{1 / 2,1 / 2}^{2-k-|\alpha|}\left(\boldsymbol{R}^{n} \times \boldsymbol{R}^{n-1}\right) .
$$


Next from Lemma 7.8 and that supp $\chi \subset$ supp $\psi_{0}$ we have

$$
\begin{aligned}
I & =\sum_{i>k} \chi a_{i k} \prod_{l \in\{i, k\}} L_{l} \\
& +\sum_{\substack{0 \leq j \leq 2 \\
|\gamma|+j=1}} D_{x}^{(j, \gamma)} \chi\left(\sum_{i>k} a_{i, k}^{\gamma, j} \prod_{\substack{l \in\{i, k\} \\
l}} L_{b}+\sum_{i=1}^{3} a_{i}^{\gamma, j} L_{\xi}\right)
\end{aligned}
$$

with some $a_{i k} \in S_{\Phi, \varphi}^{1-|\alpha|,-|\beta|}, a_{i, k}^{\gamma, j} \in S_{\Phi, \varphi}^{1-|\alpha|-j-|\gamma|,-|\beta|}, a_{i}^{\gamma, j} \in S_{\Phi, \varphi}^{2-|\alpha|-j-|\gamma|,-|\beta|}$. Here we have $D_{x}^{(j, \gamma)} \chi \circ a_{i, k}^{\gamma, j} \in S_{\Phi, \varphi}^{1-|\alpha|,-|\beta|}, D_{x}^{(j, \gamma)} \chi \circ a_{i}^{\gamma, j} \in S_{\Phi, \varphi}^{1-|\alpha|,-|\beta|-1}$.

(ii) Assume $|\alpha|+|\beta|=2$. From Corollary 5.2 we have

$$
I I+I I I+I V=\sum_{k=0}^{\min \left(2,3-a_{1}\right)} b_{k} \xi_{1}^{k} \quad \text { with some } \quad b_{k} \in S_{1 / 2,1 / 2}^{5 / 2-k-|\alpha|}\left(\mathbb{R}^{n} \times \mathbb{R}^{n-1}\right) .
$$

Similarly as in (i)

$$
I=\chi\left(\sum_{i>k} a_{i k} \prod_{l \in\{i, k\}} L_{l}+\sum_{i=1}^{3} a_{i} L_{i}\right)
$$

with $a_{i k} \in S_{\Phi, \varphi}^{1-|\alpha|,-|\beta|}, a_{i} \in S_{\Phi, \varphi}^{2-|\alpha|,-|\beta|}$. From (i) and (ii) we see using Lemma 7.1, 7.3-(1), and that $\left\langle\xi^{\prime}\right\rangle^{(|\alpha|-|\beta|) / 2} \in S_{\phi, \varphi}^{(|\alpha|-|\beta|) / 2,(|\beta|-|\alpha|) / 2}$ that

$$
\begin{aligned}
& \left\langle\xi^{\prime}\right\rangle^{(|\alpha|-|\beta|) / 2} \circ p_{(\beta)}^{(\alpha)} \circ \chi \\
& \quad=\sum_{i>j} A_{i j} \circ L_{\dot{i}} \circ L_{j}+\sum_{i=1}^{3} A_{i} \circ L_{\dot{i}}+R+\sum_{i=0}^{\min \left(2,3-\alpha_{1}\right)} B_{\dot{j}} \xi_{i}^{i}
\end{aligned}
$$

with some

$$
\begin{aligned}
& A_{i j} \in S_{\Phi, \varphi}^{1-(|\alpha|+|\beta|) / 2,-(|\alpha|+|\beta|) / 2}, R \in S_{\Phi, \varphi}^{2-(|\alpha|+|\beta|) / 2,-1-(|\alpha|+|\beta|) / 2} \\
& A_{i} \in\left\{\begin{array}{ll}
S_{\Phi, \varphi}^{1 / 2,-3 / 2} & (|\alpha|+|\beta|=1) \\
S_{\Phi, \varphi}^{1,-1} & (|\alpha|+|\beta|=2)
\end{array}, \quad B_{i} \in S_{1 / 2,1 / 2}^{3 / 2-i} .\right.
\end{aligned}
$$

This implies Lemma 3.10 in view of (3.6).

Proof of Lemma 3.11. Let $\alpha, \beta$ as in the assertion. From Lemma 5.2 we have with a notation in the claim (1) in its proof

$$
\begin{aligned}
& \left(g_{(\beta)}^{(\alpha)} \circ \chi\right)\left(x, \xi^{\prime}\right)=\sum_{\mid \gamma\}<3-|\alpha|} \frac{1}{\gamma !}\left(\partial_{\xi^{\prime}}^{\gamma}, g_{(\beta)}^{(\alpha)} D_{x^{\prime}}^{\gamma}, \chi\right)\left(x, \xi^{\prime}\right) \\
& \quad+\sum_{|\gamma|=3-|\alpha|} \frac{3-|\alpha|}{\gamma !} \int_{0}^{1} h_{\theta}\left[\partial_{\xi^{\prime}}^{\gamma} g_{(\beta)}^{(\alpha)}, D_{x^{\prime}}^{\gamma} \chi\right]\left(x, \xi^{\prime}\right)(1-\theta)^{2-|\alpha|} d \theta .
\end{aligned}
$$

The second summation on the right belongs to $S_{\Phi, \varphi}^{0,-(3-|\alpha|)} \subseteq S_{1 / 2,1 / 2}^{(3-|\alpha|) / 2}\left(\mathbb{R}^{n} \times\right.$ $\mathbb{R}^{n-1}$ ) from the claim (1) in the proof of Lemma 5.2, because $\partial_{\xi^{\prime}}^{\gamma} g_{(\beta)}^{(\alpha)} \in S_{1,0}^{0}\left(\mathbb{R}^{n} \times\right.$ $\left.\mathbb{R}^{n-1}\right) \subseteq S_{\Phi^{\prime} \varphi}^{0,0}$ if $|\gamma|+|\alpha|=3$. Next in the first summation we have $\partial_{\xi^{\prime}}^{\gamma} g_{(\beta)}^{(\alpha)} D_{x^{\prime}}^{\gamma} \chi$ $\in S_{\Phi, \varphi}^{3-|\gamma|-|\alpha|,-|\gamma|-|\beta|}$, so it belongs to $S_{\Phi, \varphi}^{3 / 2-|\gamma|-|\alpha|,-3 / 2-|\gamma|-|\mu|}$ since $\Phi\left(x, \xi^{\prime}\right)^{3} \leq$ 
$C\left\langle\xi^{\prime}\right\rangle^{3 / 2} \leq C^{\prime}(\Phi / \varphi)^{3 / 2}$ on supp $\chi$. Thus the second and first summations multiplied by $\left\langle\xi^{\prime}\right\rangle^{(|\alpha|-|\beta|) / 2}$ from the left in the operator product sence belong to respectively to $S_{1 / 2,1 / 2}^{3 / 2-1 / 1 / 2}\left(\boldsymbol{R}^{n} \times \boldsymbol{R}^{n-1}\right)$ and $S_{\phi, \varphi}^{3 / 2-|\gamma|-(|\alpha|+|\beta|) / 2,-3 / 2-|\gamma|-(|\alpha|+|\beta|) / 2} \subseteq$ $S_{1 / 2,1 / 2}^{3 / 2}\left(\boldsymbol{R}^{n} \times \boldsymbol{R}^{n-1}\right)$. This proves the assertion.

Q.E.D.

Proof of Lemma 3.12. This has been proved in the proof of Lemma 3.6.

Q.E.D.

\section{§8. Invariance of the Assumption of Theorem 1.1}

Let $\Omega_{1}=\Omega_{11} \times \Omega_{12}$ where $\Omega_{11}, \Omega_{12}$ are open sets in $\boldsymbol{R}, \boldsymbol{R}^{n-1}$ containing the origin respectively. Let $\varphi \in C^{\infty}\left(\Omega_{12}\right)$ with $\varphi(0)=0, d \varphi(0)=0$ and set $\Phi(x)=$ $\left(x_{1}-\varphi\left(x^{\prime}\right), x^{\prime}\right)$ where $x^{\prime}=\left(x_{2}, \cdots, x_{n}\right)$. Then $\Phi(0)=0$ and $\Phi$ is a diffeomorphism from $\Omega_{1}$ onto some open neighbourhood of the origin. Set $\Psi=\Phi^{-1}$ and let $\widetilde{P}(y, D)$ be a differential operator on $\Omega_{2}$ with the symbol $\widetilde{P}(y, \eta)$ defined by

$$
\widetilde{P}(y, D) u(y)=[P(x, D)(u \circ \Phi)](\Psi(y)), u \in C^{\infty}\left(\Omega_{2}\right) .
$$

Then we have

$$
\widetilde{P}\left(\Phi\left(x_{0}\right), \eta\right)=\left.\sum_{\alpha} \frac{1}{\alpha !} P^{(\alpha)}\left(x_{0},{ }^{t} \Phi^{\prime}\left(x_{0}\right) \eta\right) D_{x}^{\alpha}\left[e^{i\left\langle f\left(x, x_{0}\right), \eta\right\rangle}\right]\right|_{x=x_{0}}, x_{0} \in \Omega_{1}
$$

where $f\left(x, x_{0}\right)=\Phi(x)-\Phi\left(x_{0}\right)-\Phi^{\prime}\left(x_{0}\right)\left(x-x_{0}\right)$. If $\widetilde{P}(y, \eta)=\widetilde{P}_{m}(y, \eta)+\cdots+$ $\widetilde{P}_{0}(y, \eta)$ with $\widetilde{P}_{j}$ homogeneous of degree $j$ in $\xi$,

$$
\begin{gathered}
P_{m}\left(x,{ }^{t} \Phi^{\prime}(x) \eta\right)=\widetilde{P}_{m}(\Phi(x), \eta) \\
P_{m-1}\left(x, \Phi^{\prime}(x) \eta\right)+\sum_{|\alpha|=2} P_{m}^{(\alpha)}\left(x,{ }^{t} \Phi^{\prime}(x) \eta\right) D_{x}^{\alpha}\langle i \Phi(x), \eta\rangle / \alpha !=\widetilde{P}_{m-1}(\Phi(x), \eta) .
\end{gathered}
$$

The aim of this section is to prove the following.

Lemma 8.1. $\quad \widetilde{P}(y, D)$ satisfies the assumptions (i), (ii) in Theorem 1.1.

Proof. From the assumption (i) in Theorem 1.1, for any $\xi_{0}^{\prime} \in \boldsymbol{R}^{n-1} \backslash(0)$ there exist an open neighbourhood $U$ of the origin in $\boldsymbol{R}^{n}$ and an open conic neighbourhood $\Gamma$ of $\xi_{0}^{\prime}$ in $C^{n-1} \backslash(0)$ such that

$$
Q_{i}(x, \xi)=Q_{i}\left(x, e_{1}\right) \prod_{i=1}^{m_{i}}\left(\xi_{1}-\lambda_{i l}\left(x, \xi^{\prime}\right)\right) \quad(i=1,2)
$$

for $\left(x, \xi^{\prime}\right) \in U \times \Gamma$ as polynomials in $\xi_{1}$ where $\lambda_{i l} \in C^{\infty}(U \times \Gamma)$ which is holomorphic in $\xi^{\prime}$ and satisfies that $\lambda_{i l}\left(x, \xi^{\prime}\right) \neq \lambda_{i s}\left(x, \xi^{\prime}\right)$ for all $\left(x, \xi^{\prime}\right)$ when $l \neq s$.

Since ${ }^{t} \Phi^{\prime}(0)=i d$ and $\Phi(0)=0$, it is trivial that (i) also holds for $\widetilde{P}(y, D)$. Thus we shall show that (ii) holds for $\widetilde{P}(y, D)$. Assume that $\widetilde{P}_{m}=\partial_{\eta_{1}} \widetilde{P}_{m}=\partial_{\eta_{1}}^{2}$ 
$\widetilde{P}_{m}=0$ at $\left(0, \xi_{0}\right) \in \mathbb{R}^{n} \times(\boldsymbol{C} \backslash \mathbb{R}) \times\left(\mathbb{R}^{n-1} \backslash(0)\right)$. Then $P_{m}=\partial_{\xi_{1}} P_{m}=\partial_{\xi_{1}}^{2} P_{m}=0$ at $\left(0, \xi_{0}\right)$. This implies that $\lambda_{1 l_{1}}\left(0, \xi_{0}^{\prime}\right)=\lambda_{2 l_{2}}\left(0, \xi_{0}^{\prime}\right)=\xi_{01}$ for some $l_{1} \in\left\{1, \cdots, m_{i}\right\}$ $(i=1,2)$. Set

$$
\begin{gathered}
\lambda_{1 l_{1}}=\lambda, \quad \lambda_{1 l_{1}}-\lambda_{2 l_{2}}=c, \\
q(x, \xi)=P_{m}\left(x, e_{1}\right) \prod_{l \neq l_{1}}\left(\xi_{1}-\lambda_{1 l}\left(x, \xi^{\prime}\right)\right)^{2} \prod_{l \neq l_{2}}\left(\xi_{1}-\lambda_{2 l}\left(x, \xi^{\prime}\right)\right) .
\end{gathered}
$$

Then

$$
\begin{gathered}
P_{m}=\left(\xi_{1}-\lambda\right)^{2}\left(\xi_{1}-\lambda+c\right) q,\left(x, \xi^{\prime}\right) \in U \times \Gamma, \xi_{1} \in \mathbb{C}, \\
\lambda\left(0, \xi_{0}^{\prime}\right)=\xi_{01}, c\left(0, \xi_{0}^{\prime}\right)=0, q\left(0, \xi_{0}\right) \neq 0 .
\end{gathered}
$$

Let $\psi \in C^{\infty}\left(\Omega_{1}\right)$ with $d \psi(0)=(1,0, \cdots, 0)$. Then

$$
\begin{aligned}
& \left\{P_{m}, \psi\right\}=3\left(\psi_{x_{1}}^{\prime}-\{\lambda, \psi\}\right)\left(\xi_{1}-\lambda\right)\left(\xi_{1}-\lambda+\frac{2}{3} c\right) q \\
& \quad+\left(\xi_{1}-\lambda\right)^{2}\left(\xi_{1}-\lambda+c\right)\{q, \psi\} \\
& \quad+\left(\xi_{1}-\lambda\right)^{2}\{c, \psi\} q .
\end{aligned}
$$

Here, by definition, $\{f, g\}(x, \xi)=\sum_{j=1}^{n}\left(\partial_{\xi_{j}} f \partial_{x_{j}} g-\partial_{x_{j}} f \partial_{\xi_{j}} g\right)(x, \xi)$ for $C^{\infty}-$ functions $f, g$ in an open set of $\mathbb{R}^{n} \times \mathbb{C}^{n}$, which are holomorphic in $\xi$. Set

$$
\begin{aligned}
& F\left(\sigma, z, u,\left(x, \xi^{\prime}\right)\right)=3\left(\psi_{x_{1}}^{\prime}(x)-\{\lambda, \psi\}\left(x, \xi^{\prime}\right)\right)\left(\sigma+\frac{2}{3}\right) q\left(x,\left(\lambda\left(x, \xi^{\prime}\right)+\sigma z, \xi^{\prime}\right)\right) \\
& +\sigma(\sigma+1) z\{q, \psi\}\left(x,\left(\lambda\left(x, \xi^{\prime}\right)+\sigma z, \xi^{\prime}\right)\right) \\
& +q\left(x,\left(\lambda\left(x, \xi^{\prime}\right)+\sigma z, \xi^{\prime}\right)\right) u \sigma \\
& \text { for }(\sigma, z, u) \in \mathbb{C}^{3},\left(x, \xi^{\prime}\right) \in U \times \Gamma \text {. }
\end{aligned}
$$

$$
\begin{aligned}
& F\left(-\frac{2}{3}, 0,0,\left(x, \xi^{\prime}\right)\right)=0 \text { on } U \times \Gamma, \\
& \partial_{\sigma} F\left(-\frac{2}{3}, 0,0,\left(0, \xi_{0}^{\prime}\right)\right) \neq 0 .
\end{aligned}
$$

Thus from the implicit function theorem and the uniqueness of the implicit function, there exists a $C^{\infty}$-function $\sigma\left(z, u,\left(x, \xi^{\prime}\right)\right)$ on an open set $V=$ $V_{1} \times V_{2}$ in $\mathbb{C}^{2} \times U \times \Gamma$ with $V_{1} \subset \mathbb{C}^{2}, V_{2} \subset U \times \Gamma$ containing $\left(0,0,\left(0, \xi_{0}^{\prime}\right)\right)$ such that

$$
\begin{gathered}
F\left(\sigma\left(z, u,\left(x, \xi^{\prime}\right)\right), z, u,\left(x, \xi^{\prime}\right)\right)=0 \text { on } V_{1}, \\
\sigma\left(0,0,\left(x, \xi^{\prime}\right)\right)=-\frac{2}{3} \text { on } V_{2} .
\end{gathered}
$$

We may assume that 


$$
\left(c\left(x, \xi^{\prime}\right),\{c, \psi\}\left(x, \xi^{\prime}\right)\right) \in V_{1} \text { when }\left(x, \xi^{\prime}\right) \in V_{2} .
$$

Noting this we set

$$
a\left(x, \xi^{\prime}\right)=\sigma\left(c\left(x, \xi^{\prime}\right),\{c, \psi\}\left(x, \xi^{\prime}\right),\left(x, \xi^{\prime}\right)\right) \text { for }\left(x, \xi^{\prime}\right) \in V_{2} .
$$

Then (8.2) (8.4) and (8.6) imply that

$$
\left\{P_{m}, \psi\right\}\left(x,\left((\lambda+a c)\left(x, \xi^{\prime}\right), \xi^{\prime}\right)\right)=0 \text { on } V_{2} \text {. }
$$

Since $\sigma\left(z, u,\left(x, \xi^{\prime}\right)\right)$ is holomorphic in $(z, u),(8.5)$ implies that there exists an open subset $W$ of $V_{2}$ containing $\left(0, \xi_{0}^{\prime}\right)$ such that with some $a_{i} \in C^{\infty}(W)$ $(i=1,2)$

$$
a\left(x, \xi^{\prime}\right)=-\frac{2}{3}+c\left(x, \xi^{\prime}\right) a_{1}\left(x, \xi^{\prime}\right)+\{c, \psi\}\left(x, \xi^{\prime}\right) a_{2}\left(x, \xi^{\prime}\right) \text { on } W .
$$

Since $a\left(0, \xi_{0}^{\prime}\right)=-\frac{2}{3}$, we may assume that

$$
\left|a\left(x, \xi^{\prime}\right)+\frac{2}{3}\right|<\frac{1}{10} \text { on } W .
$$

Since $\left\{P_{m}, \psi\right\}\left(0,\left(\xi_{1}, \xi_{0}^{\prime}\right)\right)=\partial_{\xi_{1}} P_{m}\left(0,\left(\xi_{1}, \xi_{0}^{\prime}\right)\right)$, the degree of a polynomial $\left\{P_{m}, \psi\right\}(x, \xi)$ in $\xi_{1}$ is constant for $\left(x, \xi^{\prime}\right)$ in an open subset $W_{1}$ of $W$ containing $\left(0, \xi_{0}^{\prime}\right)$ where both of $\xi_{1}=\lambda\left(x, \xi^{\prime}\right)$ and $\xi_{1}=(\lambda+a c)\left(x, \xi^{\prime}\right)$ are solutions of the equation $\left\{P_{m}, \psi\right\}(x, \xi)=0$ from (8.2) and (8.7). Since $\xi_{1}=\lambda\left(x, \xi^{\prime}\right)$ is a double root of this equation for $\left(x, \xi^{\prime}\right) \in W_{1}$ with $c\left(x, \xi^{\prime}\right)=0$, and since $\lambda\left(x, \xi^{\prime}\right)$ and $(\lambda+a c)\left(x, \xi^{\prime}\right)$ are distinct for $\left(x, \xi^{\prime}\right) \in W_{1}$ with $c\left(x, \xi^{\prime}\right) \neq 0$ because of (8.9), we have that

$$
\left\{P_{m}, \psi\right\}\left(x, \xi^{\prime}\right)=\left(\xi_{1}-(\lambda+a c)\left(x, \xi^{\prime}\right)\right)\left(\xi_{1}-\lambda\left(x, \xi^{\prime}\right)\right) q_{1}(x, \xi),\left(x, \xi^{\prime}\right) \in W_{1}
$$

as polynomials in $\xi_{1}$ where $q_{1}$ is a polynomial in $\xi_{1}$ with coefficients in $C^{\infty}\left(W_{1}\right)$.

Let $\hat{W}_{1}$ be the intersection of $W_{1}$ and $\left\{\left(x, \xi^{\prime}\right) \in \boldsymbol{R}^{n} \times \boldsymbol{C}^{n-1} ;\left|\xi^{\prime}\right|=\left|\xi_{0}^{\prime}\right|\right\}$ and let $\tilde{W}_{1}$ be an open cone generated by $\hat{W}_{1}$. We extend the restrictions of functions $a, a_{1}, a_{2}$ to $\hat{W}_{1}$ to functions on $\tilde{W}_{1}$ being homogeneous degree $0,-1,0$ in $\xi^{\prime}$ respectively and we also extend the restriction of $q_{1}$ to $\boldsymbol{C} \times \hat{W}_{1}$ to function on $\boldsymbol{C} \times \tilde{W}_{1}$ being homogeneous degree $m-3$ in $\xi$. Then using homogeneity of $c$, $\{c, \psi\},\left\{P_{m}, \psi\right\}$ we see that $(8.8) \sim(8.10)$ also hold on $\tilde{W}_{1}$ when we replace $a$, $a_{1}, a_{2}, q_{1}$ by their extentions in the above. Moreover since multiplicities of the characteristic roots of $P_{m}$ are at most triple, we see from $(8.10) q_{1}\left(0,\left(\lambda\left(0, \xi_{0}^{\prime}\right)\right.\right.$, $\left.\left.\xi_{0}^{\prime}\right)\right) \neq 0$.

Thus taking $\psi(x)=\psi_{0}(x) \equiv x_{1}-\varphi\left(x^{\prime}\right)$ and $\psi(x)=x_{1}$ we see that there exist 
an open subset $U_{1}$ of $U$ containing the origin, an open conic subset $\Gamma_{1}$ of $\Gamma$ containing $\xi_{0}^{\prime}$, and an open conic subset $\tilde{\Gamma}$ of $C \times \Gamma$ containing $\left(\lambda\left(0, \xi_{0}^{\prime}\right), \xi_{0}^{\prime}\right)$ such that the following factorization of $\partial_{\xi_{1}} P_{m}$ and $\left\{P_{m}, \psi_{0}\right\}$ holds:

$$
\begin{aligned}
& \partial_{\xi_{1}} P_{m}(x, \xi)=\left(\xi_{1}-\left(\lambda+a_{0} c\right)\left(x, \xi^{\prime}\right)\right)\left(\xi_{1}-\lambda\left(x, \xi^{\prime}\right)\right) \tilde{q}(x, \xi), \\
& \left\{P_{m}, \psi_{0}\right\}(x, \xi)=\left(\xi_{1}-(\lambda+b c)\left(x, \xi^{\prime}\right)\right)\left(\xi_{1}-\lambda\left(x, \xi^{\prime}\right)\right) \tilde{q}(x, \xi)
\end{aligned}
$$

for $\left(x, \xi^{\prime}\right) \in U_{1} \times \Gamma_{1}$ as polynomials in $\xi_{1}$ where $a_{0}, b \in C^{\infty}\left(U_{1} \times \Gamma_{1}\right)$ and $\tilde{q}, \tilde{q} \in$ $C^{\infty}\left(U_{1} \times \Gamma\right)$ satisfying that

$$
\begin{aligned}
& \left|a_{0}+\frac{2}{3}\right|<\frac{1}{10} ; \\
& a_{0}=-\frac{2}{3}+c a_{01}, \\
& b=-\frac{2}{3}+c b_{1}+\left\{c, \psi_{0}\right\} b_{2}
\end{aligned}
$$

with some $a_{01}, b_{1}, b_{2} \in C^{\infty}\left(U_{1} \times \Gamma_{1}\right)$ which are homogeneous degree $-1,-1,0$ in $\xi^{\prime}$ respectively; $q$ and $\tilde{q}$ are homogeneous degree $m-3$, and

$$
C|\tilde{q}(x, \xi)| \geq|\xi|^{m-3} \text { and } C|\tilde{\tilde{q}}(x, \xi)| \geq|\xi|^{m-3} \text { on } U_{1} \times \tilde{\Gamma}
$$

for some positive constant $C$.

We may assume, decreasing $U_{1}$ and $\tilde{\Gamma}$ if necessary, that

the inequality in the assumption (ii) in Theorem 1.1 holds when $(x, \xi) \in U_{1} \times \tilde{\Gamma}$ and $\partial_{\xi_{1}} P_{m}(x, \xi)=0$;

$$
C|q(x, \xi)| \geq|\xi|^{m-3} \text { on } U_{1} \times \tilde{\Gamma} \text { for some } C>0 .
$$

We define $\tau_{0}, \tau \in C^{\infty}\left(U_{1} \times \Gamma_{1}\right)$ by

$\tau_{0}\left(x, \xi^{\prime}\right)=\left(\lambda+a_{0} c\right)\left(x, \xi^{\prime}\right)$ and $\tau\left(x, \xi^{\prime}\right)=(\lambda+b c)\left(x, \xi^{\prime}\right)$ for $\left(x, \xi^{\prime}\right) \in U_{1} \times \Gamma_{1}$.

To prove that the assumption (ii) holds for $\widetilde{P}(x, D)$ we must show that there exists an open conic neighbourhood $\tilde{\tilde{\Gamma}} \subset \tilde{\Gamma}$ of $\left(0, \xi_{0}\right)$ in $\mathbb{R}^{n} \times C^{n}$ such that

$$
\begin{aligned}
& \left(\left|\left(\partial_{\eta} \widetilde{P}_{m}\right)(y, \eta)\right||\eta|+\left|\left(\partial_{y} \widetilde{P}_{m}\right)(y, \eta)\right|\right)\left|\widetilde{P}_{m-1}(y, \eta)\right| \\
& \quad \leq C\left|\widetilde{P}_{m}(y, \eta)\right|^{2 / 3}\left(\left|P_{m}(y, \eta)\right|^{1 / 3}|\eta|^{m-1}+\left|\left(\widetilde{P}_{m}+\widetilde{P}_{m-1}\right)(y, \eta)\right||\eta|^{m / 3}\right. \\
& \left.\quad+|\eta|^{(4 m / 3)-(3 / 2)}+1\right)
\end{aligned}
$$

From $(8,1)$ it is easy to see that $(8.16)$ follows if we prove that there exists an 
open conic neighbourhood $\tilde{\tilde{\Gamma}} \subset \tilde{\Gamma}$ of $\left(0, \xi_{0}\right) \in \boldsymbol{R}^{n} \times \boldsymbol{C}^{n}$ such that

$$
\begin{aligned}
\left(\left|\left(\partial_{\xi} P_{m}\right)(x, \xi)\right||\xi|+\left|\left(\partial_{x} P_{m}\right)(x, \xi)\right|\right)\left(\left|P_{m-1}(x, \xi)\right|+\sum_{|\alpha|=2}\left|P_{m}^{(\alpha)}(x, \xi)\right||\xi|\right) \\
\leq \mid \\
\quad+\mid\left(P _ { m } ( x , \xi ) | ^ { 2 / 3 } \left(\left|P_{m}(x, \xi)\right|^{1 / 3}|\xi|^{m-1}\right.\right. \\
\left.\quad+|\xi|^{(4 m / 3)-(3 / 2)}\right)(x, \xi)+\sum_{|\alpha|=2} P_{m}^{(\alpha)}(x, \xi) D_{x}^{\alpha}<i \Phi(x),{ }^{t} \Phi^{\prime}(x)^{-1} \xi>|\alpha !||\xi|^{m / 3} \\
\quad
\end{aligned}
$$

when $(x, \xi) \in \tilde{\tilde{\Gamma}}$ and $\left\{P_{m}, \psi_{0}\right\}(x, \xi)=0$. Note that (8.11), (8.13), and the definition of $\tau\left(x, \xi^{\prime}\right)$ imply that

$$
\left\{P_{m}, \psi_{0}\right\}=0 \text { if and only if } \xi_{1}=\lambda\left(x, \xi^{\prime}\right) \text { or } \xi_{1}=\tau\left(x, \xi^{\prime}\right)
$$

when $\left(x, \xi^{\prime}\right) \in \tilde{\Gamma}$.

Thus since the inequality in (8.17) is trivial when $(x, \xi) \in \tilde{\Gamma}$ and $\xi_{1}=$ $\lambda\left(x, \xi^{\prime}\right)$, it suffices for us to show that

there exists an open neighbourhood $U_{0} \subset U_{1}$ of the origin in $\boldsymbol{R}^{n}$

an open conic neighbourhood $\Gamma_{0} \subset \Gamma_{1}$ of $\xi_{0}^{\prime}$ in $\boldsymbol{C}^{n-1}$ such that the inequality in (8.17) holds when $\left(x, \xi^{\prime}\right) \in U_{0} \times \Gamma_{0}$ and $\xi_{1}=\tau\left(x, \xi^{\prime}\right)$.

Indeed, if (8.18) is proved, (8.17) holds with $\tilde{\tilde{\Gamma}}=\tilde{\Gamma} \cap\left(U_{0} \times\left(\boldsymbol{C} \times \Gamma_{0}\right)\right)$.

We shall show (8.18). Let us choose an open neighbourhood $U_{2}$ of the origin in $\boldsymbol{R}^{n}$ and an open conic neighbourhood $\Gamma_{2}$ of $\xi_{0}^{\prime}$ in $\boldsymbol{C}^{n-1} \backslash(0)$ so that

$$
\begin{gathered}
U_{2} \subset \subset U_{1}, \Gamma_{2} \cap\left\{\xi^{\prime} \in C^{n-1} ;\left|\xi^{\prime}\right|=1\right\} \subset \subset \Gamma_{1} ; \\
\left(\tau_{0}\left(x, \xi^{\prime}\right), \xi^{\prime}\right),\left(\tau\left(x, \xi^{\prime}\right), \xi^{\prime}\right) \in \tilde{\Gamma} \text { when }\left(x, \xi^{\prime}\right) \in U_{2} \times \Gamma_{2} .
\end{gathered}
$$

Sublemma 8.1. The following estimates holds on $U_{2} \times \Gamma_{2}$.

$$
\begin{gathered}
\left.C\left|P_{m}\right|\right|_{\xi_{1}=\tau_{0}} \geq|c|^{3}\left|\xi^{\prime}\right|^{m-3} \geq\left. C^{-1}\left|P_{m}\right|\right|_{\xi_{1}=\tau_{0}} \\
\left|\left(P_{m}\right)_{(\alpha)}^{(\beta)}\right|_{\xi_{1}=\tau}-\left.\left.\left(P_{m}\right)_{(\alpha)}^{(\beta)}\right|_{\xi_{1}=\tau_{0}}\left|\leq C\left(|c|\left|\xi^{\prime}\right|^{-1}+\left|\left\{c, \psi_{0}\right\}\right|\right)\right| c\right|^{3-|\alpha|-|\beta|}\left|\xi^{\prime}\right|^{m-3+|\alpha|}
\end{gathered}
$$

if $|\alpha|+|\beta| \leq 2$.

$$
\left.\left|\left(P_{m}\right)_{(\alpha)}^{(0, \beta)}\right|\left|\xi_{1=\tau_{0}} \geq C_{1}\right| c_{(\alpha)}^{(\beta)}|| c\right|^{2}\left|\xi^{\prime}\right|^{m-3}-C_{2}|c|^{3}\left|\xi^{\prime}\right|^{m-3-|\beta|}
$$

if $\alpha \in \mathbb{Z}_{+}^{n}, \beta \in \mathbb{Z}_{+}^{n-1}$ with $|\alpha|+|\beta|=1$. Here, constants $C, C_{1}, C_{2}$ are all positive.

Proof of Sublemma 8.1. (8.21) immediately follows from (8.12). To show the next two inequalities we observe that 


$$
\left.\left|\left(P_{m}\right)_{(\alpha)}^{(\beta)}\right|\left|\xi_{1=\tau_{0}} \leq C\right| \xi^{\prime}\right|^{m-3+|\alpha|}|c|^{3-|\alpha|-|\beta|} \text { on } U_{2} \times \Gamma_{2}
$$

if $|\alpha|+|\beta| \leq 2$.

$$
\left|\tau-\tau_{0}\right| \leq C\left(|c|\left|\xi^{\prime}\right|^{-1}+\left|\left\{c, \psi_{0}\right\}\right|\right)|c| \text { on } U_{2} \times \Gamma_{2} .
$$

Then we obtain (8.22) by Tayolr expansion of $\left(\boldsymbol{P}_{m}\right)_{(\boldsymbol{\alpha})}^{(\boldsymbol{\beta})}$ in $\xi_{1}$ at $\xi_{1}=\tau_{0}$, substituting $\tau$ for $\xi_{1}$, and estimating each term in the expansion by (8.24) and (8.25) except for $\left.\left(P_{m}\right)_{(\alpha)}^{(\beta)}\right|_{\xi_{1}=\tau_{0}}$. Finally (8.23) immediately follows from (8.12) and the equality that for $\alpha, \beta$ as in (8.23).

$$
\begin{aligned}
& \left(P_{m}\right)_{(\alpha)}^{(0, \beta)}=-3 \lambda_{(\alpha)}^{(\beta)}\left(\xi_{1}-\lambda\right)\left(\xi_{1}-\lambda+\frac{2}{3} c\right) q+c_{(\alpha)}^{(\beta)}\left(\xi_{1}-\lambda\right)^{2} q \\
& \quad+\left(\xi_{1}-\lambda\right)^{2}\left(\xi_{1}-\lambda+c\right) q_{(\alpha)}^{(0, \beta)} .
\end{aligned}
$$

Q.E.D.

Since $c=\left\{c, \psi_{0}\right\}=0$ at $\left(0, \xi_{0}^{\prime}\right)$, from (8.21), (8.22), and (8.25) there exists an open nighbourhood $U_{3} \subset U_{2}$ of the origin in $\mathbb{R}^{n}$ and an open conic neighbourhood $\Gamma_{3} \subset \Gamma_{2}$ of $\xi_{0}^{\prime}$ in $\mathbb{C}^{n}$ such that for some positive constant $C$

$$
\left.C\left|P_{m}\right|\right|_{\xi_{1}=\tau} \geq|c|^{3}\left|\xi^{\prime}\right|^{m-3} \geq\left. C^{-1}\left|P_{m}\right|\right|_{\xi_{1}=\tau} \text { on } U_{3} \times \Gamma_{3} .
$$

From (8.25) we have that

$$
\begin{aligned}
& \left|P_{m-1}\right|_{\xi_{1}=\tau}-\left.P_{m-1}\right|_{\xi_{1}=\tau_{0}} \mid \\
& \quad \leq C\left(|c|\left|\xi^{\prime}\right|^{-1}+\left|\left\{c, \psi_{0}\right\}\right|\right)|c|\left|\xi^{\prime}\right|^{m-2} \text { on } U_{2} \times \Gamma_{2} .
\end{aligned}
$$

Using (8.21), (8.22), (8.24), (8.26), (8.27) we see that on $U_{3} \times \Gamma_{3}$

$$
\begin{aligned}
& \left.\left(\left|\partial_{\xi} P_{m}\right||\xi|+\left|\partial_{x} P_{m}\right|\right)\left(\left|P_{m-1}\right|+\sum_{|\alpha|=2}\left|P_{m}^{(\alpha)}\right||\xi|\right)\right|_{\xi_{1}=\tau} \\
& \quad \leq\left. C\left\{\left(\left|\partial_{\xi} P_{m}\right||\xi|+\left|\partial_{x} P_{m}\right|\right)\left|P_{m-1}\right|+\left|P_{m}\right||\xi|^{m-1}\right\}\right|_{\xi_{1}=\tau_{0}} \\
& \left.\left|P_{m}\right|^{2 / 3} \sum_{|\alpha|=2}\left|P_{m}^{(\alpha)}\right||\xi|^{(m / 3)+1}\right|_{\xi_{1}=\tau} \leq\left. C\left|P_{m}\right||\xi|^{m-1}\right|_{\xi_{1}=\tau}
\end{aligned}
$$

In the same way one can deduce that on $U_{3} \times \Gamma_{3}$

$$
\begin{aligned}
& \left.\left|P_{m}\right|^{2 / 3}\left|P_{m}+P_{m-1}\right||\xi|^{m / 3}\right|_{\xi_{1}=\tau_{0}} \\
& \leq\left. C_{1}\left|P_{m}\right|^{2 / 3}\left|P_{m}+P_{m-1}\right||\xi|^{m / 3}\right|_{\xi_{1}=\tau} \\
& \quad+\left.C_{2}\left(|c|\left|\xi^{\prime}\right|^{-1}+\left\{c, \psi_{0}\right\} \mid\right)\left(|c|^{3}\left|\xi^{\prime}\right|^{m-3}\right)^{2 / 3}\left(\left|P_{m}\right||\xi|^{m / 3}\right)\right|_{\xi_{1}=\tau} \\
& \quad+\left.C_{3}\left(|c|\left|\xi^{\prime}\right|^{-1}+|\{c, \psi\}|\right)\left|\xi^{\prime}\right|^{m-1}\left|P_{m}\right|\right|_{\xi_{1}=\tau} \\
& \leq\left. C_{4}\left|P_{m}\right|^{2 / 3}\left|P_{m}+P_{m-1}\right||\xi|^{m / 3}\right|_{\xi_{1}=\tau} \\
& \quad+\left.C_{2}\left(|c|\left|\xi^{\prime}\right|^{-1}+\left|\left\{c, \psi_{0}\right\}\right|\right)|c|^{2}\left|\xi^{\prime}\right|^{m-2}\left|P_{m-1}\right|\right|_{\xi_{1}=\tau}+\left.C_{5}\left|P_{m}\right||\xi|^{m-1}\right|_{\xi_{1}=\tau}
\end{aligned}
$$

From (8.23) and (8.26) the middle term on the right of the second inequlaity can be dominated on $U_{3} \times \Gamma_{3}$ by a constant multiple of 


$$
\left.\left|P_{m}\right||\xi|^{m-1}\right|_{\xi_{1}=\tau}+\left.\left|d \psi_{0}\right||\xi|\left|\partial_{\xi} P_{m}\right|\left|P_{m-1}\right|\right|_{\xi_{1}=\tau_{0}} .
$$

Thus we get with another constants

$$
\begin{aligned}
& \left.\left|P_{m}\right|^{2 / 3}\left|P_{m}+P_{m-1}\right||\xi|^{m / 3}\right|_{\xi_{1}=\tau_{0}} \\
& \quad \leq\left. C_{1}\left(\left|P_{m}\right|^{2 / 3}\left|P_{m}+P_{m-1}\right||\xi|^{m / 3}+\left|P_{m}\right||\xi|^{m-1}\right)\right|_{\xi_{1}=\tau} \\
& \quad+\left.C_{2}\left|d \psi_{0}\right||\xi|\left|\partial_{\xi} P_{m}\right|\left|P_{m-1}\right|\right|_{\xi_{1}=\tau_{0}}
\end{aligned}
$$

on $U_{3} \times \Gamma_{3}$

From (8.11) and (8.14) the inequality in (ii) in Theorem 1.1 holds when $\left(x, \xi^{\prime}\right) \in U_{3} \times \Gamma_{3}$ and $\xi_{1}=\tau_{0}$. Thus conbining this inequality, (8.21), (8.26) (8.30) we obtain

$$
\begin{aligned}
& \left.\left(\left|\partial_{\xi} P_{m}\right||\xi|+\left|\partial_{x} P_{m}\right|\right)\left(\left|P_{m-1}\right|+\sum_{|\alpha|=2}\left|P_{m}^{(\alpha)}\right||\xi|\right)\right|_{\xi_{1}=\tau} \\
& \leq\left. C_{1}\left\{\left(\left|\partial_{\xi} P_{m}\right||\xi|+\left|\partial_{x} P_{m}\right|\right)\left|P_{m-1}\right|+\left|P_{m}\right||\xi|^{m-1}\right\}\right|_{\xi_{1}=\tau_{0}} \\
& \leq C_{2}\left\{\left.\left|P_{m}\right|^{2 / 3}\left(\left|P_{m}\right|^{1 / 3}|\xi|^{m-1}+\left|P_{m}+P_{m-1}\right||\xi|^{m / 3}+|\xi|^{4 m / 3-3 / 2}+1\right\}\right|_{\xi_{1}=\tau}\right. \\
& \quad+\left.C_{3}|d \psi||\xi|\left|\partial_{\xi} P_{m}\right|\left|P_{m-1}\right|\right|_{\xi_{1}=\tau_{0}}
\end{aligned}
$$

on $U_{3} \times \Gamma_{3}$.

Moreover we see from (8.29) that on $U_{3} \times \Gamma_{3}$

$$
\begin{aligned}
& \left.\left|P_{m}\right|{ }^{2 / 3}\left|P_{m}+P_{m-1}\right||\xi|^{m / 3}\right|_{\xi_{1}=\tau} \\
& \quad \leq\left.\left. C_{1}\left|P_{m}\right|^{2 / 3}\left|P_{m}+P_{m-1}+\sum_{|\alpha|=2} P_{m}^{(\alpha)} D_{x}^{\alpha}<i \Phi(x),{ }^{t} \Phi^{\prime}(x)^{-1} \xi>\right| \alpha !|| \xi\right|^{m / 3}\right|_{\xi_{1}=\tau} \\
& \quad+\left.C_{2}\left|P_{m}\right||\xi|^{m-1}\right|_{\xi_{1}=\tau} .
\end{aligned}
$$

This inequality, (8.31), and that $d \psi(0)=0$ immediately imply that there exists an open neighbourhood $U_{4} \subset U_{3}$ of the origin such that the inequality in (8.17) holds when $\left(x, \xi^{\prime}\right) \in U_{4} \times \Gamma_{3}$ and $\xi_{1}=\tau$. Thus, the proof of Lemma 8.1 is complete.

\section{§9. Proof of Theorem 1.1}

From Lemma 8.1, to prove Theorem 1.1 it suffices to show the existence of an open neighbourhood $\Omega^{\prime} \subset \Omega$ of the origin such that every $u \in C^{\infty}(\Omega)$ satisfy-

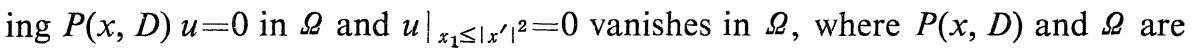
as in Theorem 1.1.

In case that $m_{1}=0$ or $m_{2}=0$ Theorem 1.1 was proved by Calderón [2], Mizohata [5], and Hörmander [4]. Thus we only have to prove Theorem 1.1 in case that $m_{1} \geq 1$ and $m_{2} \geq 1$. In this case the theorem follows from the following. 
Lemma 9.1. Let $P(x, D)$ and $\Omega$ be as in Theorem 1.1. Assume that $m_{i} \geq$ $1(i=1,2)$. Then there exist positive constants $\delta_{0}, \tau_{0}, C_{0}$ such that when $\tau T^{2}>\tau_{0}$ and $T^{-1}>\tau_{0}$,

$$
\|u\|_{m, T}^{(\tau)} \leq C_{0}\|P(x, D) u\|_{T}^{(\tau)}, u \in C_{0}^{\infty}\left(B_{\delta_{0}}(0)\right) \cap \mathcal{S}_{T / 2}\left(\mathbb{R}^{n}\right) .
$$

Here, $B_{r}(0)$ is the open ball with the center at the origin in $\mathbb{R}^{n}$ and the radius $r$, and by definition

$$
\begin{gathered}
\|u\|_{s, T}^{(\tau)}=\sum_{\substack{i+2 j \leq 2 s \\
i, j \in \overline{\boldsymbol{Z}}_{+}}} \tau^{m-3 / 2-i / 2-j} T^{m-3-i / 2-j}\left\|E_{i / 2} D_{1}^{j} u\right\|_{T}^{(\tau)} \\
\text { for } u \in \mathcal{S}\left(\mathbb{R}^{n}\right), s=0, \cdots, m .
\end{gathered}
$$

Proof. We may assume that $Q_{i}\left(x, e_{1}\right)=1(i=1,2)$, for $P_{m}\left(x, e_{1}\right)^{-1} P(x, D)$ also satisfies the assumption in Theorem 1.1.

Let $\xi_{0}^{\prime} \in \mathbb{R}^{n-1} \backslash(0)$. Then the possible cases are the following (i), (ii).

Case (i). Two equations $Q_{i}\left(0, \tau, \xi_{0}^{\prime}\right)=0(i=1,2)$ have no common root.

Then there exist an open neighbourhood $U$ of the origin in $\mathbb{R}^{n}$ and an open conic neighbourhood $\Gamma$ of $\xi_{0}^{\prime}$ in $\mathbb{R}^{n-1} \backslash 0$ such that in $U \times \Gamma$ we can write

$$
P_{m}(x, \xi)=\prod_{j=1}^{m_{1}}\left(\xi_{1}-\lambda_{j}\left(x, \xi^{\prime}\right)\right)^{2} \prod_{j=m_{1}+1}^{m_{1}+m_{2}}\left(\xi_{1}-\lambda_{j}\left(x, \xi^{\prime}\right)\right) .
$$

Here $\lambda_{j} \in C^{\infty}(U \times \Gamma),\left(1 \leq j \leq m_{1}+m_{2}\right)$ are homogeneous degree 1 in $\xi^{\prime}$ being at every point, pairwise distinct and non-real. Choose a $C^{\infty}$-mapping $\Xi\left(\xi^{\prime}\right)$ from $\mathbb{R}^{n-1}$ to $\Gamma$ such that $\Xi\left(\xi^{\prime}\right)=\xi^{\prime}$ if $\xi^{\prime}$ lies in a conic nieghbourhood of $\xi_{0}^{\prime}$ and $\left|\xi^{\prime}\right|>1$, and such that $\Xi\left(\xi^{\prime}\right)$ is homogeneous degree 1 in $\xi^{\prime}$ when $\left|\xi^{\prime}\right|>1$ and satisfies that $\left|\Xi\left(\xi^{\prime}\right)\right| \geq C\left(1+\left|\xi^{\prime}\right|\right)$. Let $\psi \in C^{\infty}\left(\mathbb{R}^{n}\right)$ with supp $\psi \subset U$, $\psi=1$ in a neighbourhood of $0,0 \leq \psi \leq 1$. Set $\Psi(x)=\psi(x) x$. We set $\widetilde{P}_{m}(x, \xi)$ $=P_{m}\left(\Psi(x),\left(\xi_{1}, \Xi\left(\xi^{\prime}\right)\right)\right), \tilde{\lambda}_{j}\left(x, \xi^{\prime}\right)=\lambda_{j}\left(\Psi(x), \Xi\left(\xi^{\prime}\right)\right)$. Then $\tilde{\lambda}_{j} \in S_{1,0}^{1}\left(\mathbb{R}^{n} \times \mathbb{R}^{n-1}\right)$, $C\left|\operatorname{Im} \tilde{\lambda}_{j}\left(x, \xi^{\prime}\right)\right| \geq 1+\left|\xi^{\prime}\right|$, and $\lambda_{j}\left(x, \xi^{\prime}\right)=\tilde{\lambda}_{j}\left(x, \xi^{\prime}\right)$ and $\widetilde{P}_{m}(x, \xi)=P_{m}(x, \xi)$ for $\left(x, \xi^{\prime}\right)$ in a conic neighbourhood of $\left(0, \xi_{0}^{\prime}\right)$.

Case (ii). Two euqations $Q_{i}\left(0, \tau, \xi_{0}^{\prime}\right)=0(i=1,2)$ have exactly $r(\geq 1)$ common roots.

Then there exist an open neighboruhood $U$ of the origin in $\mathbb{R}^{n}$ and an open conic neighbourhood $\Gamma$ of $\xi_{0}^{\prime}$ in $\mathbb{R}^{n-1} \backslash(0)$ such that in $U \times \Gamma$ we can write

$$
P_{m}(x, \xi)=\prod_{j=1}^{m_{1}+m_{2}-r} p_{j}(x, \xi)
$$

where 


$$
p_{j}(x, \xi)= \begin{cases}\left(\xi_{1}-\lambda_{j}\left(x, \xi^{\prime}\right)\right)^{2}\left(\xi_{1}-\lambda_{j}\left(x, \xi^{\prime}\right)+c_{j}\left(x, \xi^{\prime}\right)\right) & (1 \leq j \leq r) \\ \left(\xi_{1}-\lambda_{j}\left(x, \xi^{\prime}\right)\right)^{2} & \left(r<j \leq m_{1}\right) \\ \xi_{1}-\lambda_{j}\left(x, \xi^{\prime}\right) & \left(m_{1}<j \leq m_{1}+m_{2}-r\right)\end{cases}
$$

with $\lambda_{j}, c_{j} \in C^{\infty}(U \times \Gamma)$ which are homogeneous degree 1 and satisfy that $\lambda_{i}\left(1 \leq j \leq m_{1}+m_{2}-r\right)$ are non-real and distinct everywhere, $\lambda_{j}$ and $\lambda_{i}-c_{i}$ are distinct everywhere if $i \neq j$, and $\left|\operatorname{Im} \lambda_{j}\right| \geq 2\left|c_{j}\right|$ for $j=1, \cdots, r$. Since equations $p_{j}(x, \xi)=0$ in $\xi_{1}$ have no common zero for any $\left(x, \xi^{\prime}\right) \in U \times \Gamma$, we can write

$$
\begin{gathered}
P_{m-1}(x, \xi)=\sum_{i=1}^{m_{1}+m_{2}-r} q_{i}(x, \xi) \prod_{j \neq i} p_{j}(x, \xi), \\
\left(P_{m}+P_{m-1}\right)(x, \xi)=\prod_{i=1}^{m_{1}+m_{2}-r}\left(p_{i}(x, \xi)+q_{i}(x, \xi)\right)+s(x, \xi)
\end{gathered}
$$

where with the notation that $l_{i}=$ the degree of $p_{i}$ as polynomial in $\xi_{1}, q_{i}$ is a polynomial in $\xi_{1}$ of degree $l_{i}-1$ with coefficients in $C^{\infty}(U \times \Gamma)$ and homogeneous degree $l_{i}-1$ in $\xi$, and $s$ is a polynomial in $\xi_{1}$ of degree $m-2$ such that the coefficient of $\xi_{1}^{k}$ is a sum of functions in $C^{\infty}(U \times \Gamma)$ which are homogeneous in $\xi^{\prime}$ of degree $k, \cdots, k+2-\min (r, k+2)$.

Then there exist an open subset $U_{1}$ of $U$ containing the origin and an open conic subset $\Gamma_{1}$ of $\Gamma$ containing $\xi_{0}^{\prime}$ such that for any $i=1, \cdots, r$ we have that

$$
\begin{aligned}
& \quad\left|q_{i}(x, \xi)\right|\left(\left|\partial_{x} p_{i}(x, \xi)\right|+\left|\partial_{\xi} p_{i}(x, \xi)\right||\xi|\right) \\
& \quad \leq C\left|p_{i}(x, \xi)\right|^{2 / 3}\left(\left|p_{i}(x, \xi)\right|^{1 / 3}|\xi|^{2}+\left|\left(p_{i}+q_{i}\right)(x, \xi)\right||\xi|+|\xi|^{5 / 2}+1\right) \\
& \text { if } \quad(x, \xi) \in U_{1} \times\left(C \times \Gamma_{1}\right) \text { and } \quad \partial_{\xi_{1}} p_{i}(x, \xi)=0 .
\end{aligned}
$$

Indeed, from the proof of Lemma 8.1 there exist an open neighbourhood $U_{1}$ of the origin in $\boldsymbol{R}^{n}$ with $U_{1} \subset \subset U$ and an open conic neighbourhood $\Gamma_{1}$ of $\xi_{0}^{\prime}$ in $\boldsymbol{R}^{n-1} \backslash(0)$ with $\Gamma_{1} \cap S^{n-2} \subset \subset \Gamma$ such that for any $i=1, \cdots, r$ there exists $a_{i} \in C^{\infty}\left(U_{1} \times \Gamma_{1}\right)$ which is homogeneous degree 0 in $\xi^{\prime}$ and satisfying the following:

$$
a_{i}\left(0, \xi_{0}^{\prime}\right)=-\frac{2}{3}
$$

with the notation that $\tau_{i}=\lambda_{i}+a_{i} c_{i}$, the inequality

in the assumption (ii) in Theorem 1.1 holds when $\left(x, \xi^{\prime}\right) \in U_{1} \times \Gamma_{1}$ and $\xi_{1}=\tau_{i}\left(x, \xi^{\prime}\right)$, and the inequalities (8.21) and (8.23) hold on $U_{1} \times \Gamma_{1}$ with instead of $\tau_{0}$.

From (9.4) and (9.5) we may assume that on $U_{1} \times \Gamma_{1}$ 


$$
\begin{aligned}
& \left.C\left|p_{i}\right|\right|_{\xi_{1}=\tau_{j}} \geq\left|\xi^{\prime}\right|_{i} \text { if } i \neq j, \\
& C\left|c_{i}\right|^{3} \geq\left.\left|p_{i}\right|\left|\xi_{1}=\tau_{i} \geq C^{-1}\right| c_{i}\right|^{3}
\end{aligned}
$$

Using (9.5) and (9.6) one can easily see that for any $i=1, \cdots, r$ on $U_{1} \times \Gamma_{1}$

$$
\begin{aligned}
& \left.\left(\left|\partial_{x} c_{i}\right|+\left|\partial_{\xi^{\prime}} c_{i}\right|\left|\xi^{\prime}\right|\right)\left|c_{i}\right|^{2}\left|q_{i}\right|\right|_{\xi_{1}=\tau_{i}} \\
& \quad \leq C\left|c_{i}\right|^{2}\left(\left|c_{i}\right|\left|\xi^{\prime}\right|^{2}+\left.\left|p_{i}+q_{i}\right|\right|_{\xi_{1}=\tau_{i}}\left|\xi^{\prime}\right|+\left(1+\left|\xi^{\prime}\right|\right)^{5 / 2}\right) .
\end{aligned}
$$

Note that with notation that $\tau_{i 0}=\lambda_{i}-\frac{2}{3} c_{i}$

$$
\partial_{\xi_{1}} p_{i}=3\left(\xi_{1}-\lambda_{i}\right)\left(\xi_{1}-\tau_{i 0}\right)
$$

Then using this inequality and that $\left.\left(p_{i}\right)_{(0, \beta)}^{(\alpha)}\right|_{\xi_{1}=\tau_{i 0}}=\frac{4}{9}\left(c_{i}\right)_{(\beta)}^{(\alpha)} c_{i}^{2}$ if $\alpha \in \mathbb{Z}_{+}^{n}, \beta \in$ $\mathbb{Z}_{+}^{n-1}$ with $|\alpha|+|\beta|=1$, and using Taylor expansion of $p_{i}$ and $q_{i}$ in $\xi_{1}$ at $\xi_{1}=\tau_{i 0}$ we obtain (9.3) from (9.7). Let us choose mappings $\Xi\left(\xi^{\prime}\right)$ and $\Psi(x)$ as in (i) for $\Gamma_{1}$ and $U_{1}$ instead of $\Gamma$ and $U$. Then we define $\widetilde{P}_{m}, \widetilde{P}_{m-1}, \tilde{\lambda}_{j}, \tilde{c}_{j}, \tilde{p}_{j}$, $\tilde{q}_{j}, \tilde{s}$ as in the same way in case (i). Then (9.2) holds for $\left(x, \xi^{\prime}\right)$ in a conic neighbourhood of $\left(0, \xi_{0}^{\prime}\right)$ with $\tilde{p}_{j}, \tilde{q}_{j}, \tilde{s}$ instead of $p_{j}, q_{j}, s$, and $\tilde{p}_{j}$ and $\tilde{q}_{j}$ satisfy the assumption for $p$ and $q$ respectively in Proposition 1.1.

Now we prove the following lemma.

Lemma 9.2. Assume notations in the above arguments. Then we have the following estimate in the above cases (i), (ii),

Case (i). If $\tau T^{2}$ and $T^{-1}$ are large, for $u \in \mathcal{S}_{T / 2}\left(\mathbb{R}^{n}\right)$

$$
\begin{aligned}
& \tau^{1 / 2} T\|u\|_{m, T}^{(\tau)}+T^{-1 / 2} \sum_{|\alpha|=1}\left(\left\|E_{1 / 2}\left(\widetilde{P}_{m}\right)^{(\alpha)}(x, D) u\right\|_{T}^{(\tau)}+\left\|E_{-1 / 2}\left(\widetilde{P}_{m}\right)_{(\alpha)}(x, D) u\right\|_{T}^{(\tau)}\right) \\
& \quad \leq C\left\|\widetilde{P}_{m}(x, D) u\right\|_{T}^{(\tau)} .
\end{aligned}
$$

Case (ii). If $\tau T^{2}$ and $T^{-1}$ are large, for $u \in S_{T / 2}\left(\mathbb{R}^{n}\right)$

$$
\begin{aligned}
& \|u\|_{m, T}^{(\tau)}+T^{-1 / 2} \sum_{|\alpha|=1}\left(\left\|E_{1 / 2} Q^{(\alpha)}(x, D) u\right\|_{T}^{(\tau)}+\left\|E_{-1 / 2} Q_{(\alpha)}(x, D) u\right\|_{T}^{(\tau)}\right) \\
& \quad \leq C\|Q(x, D) u\|_{T}^{(\tau)} .
\end{aligned}
$$

where $Q(x, \xi)=\prod_{i=1}^{m_{1}+m_{2}-r}\left(\tilde{p}_{i}+\tilde{q}_{i}\right)(x, \xi)$.

Proof of Lemma 9.2. First we prove the estimate in case (i). The inequlaity for $\|u\|_{m, T}^{(\tau)}$ is well-known. (See [8]). We shall show that the one for $\left(\widetilde{P}_{m}\right)_{(\alpha)}$. This also contains nothing new.

Set $\tilde{Q}_{j}(x, \xi)=\left(\xi_{1}-\lambda_{j}\left(x, \xi^{\prime}\right)\right)^{2}$ for $1 \leq j \leq m_{1}, \tilde{Q}_{j}(x, \xi)=\xi_{1}-\lambda_{j}\left(x, \xi^{\prime}\right)$ for 
$m_{1}+1 \leq j \leq m_{1}+m_{2}$.

From Proposition 3.2 we have that if $\tau T^{2}$ and $T^{-1}$ are large,

$$
T^{-1 / 2} \sum_{|\alpha|+|\beta|=1}\left\|E_{(|\alpha|-|\beta|) / 2}\left(\tilde{Q}_{i}\right)_{(\beta)}^{(\alpha)}(x, D) u\right\|_{T}^{(\tau)} \leq C\left\|\tilde{Q}_{i}(x, D) u\right\|_{T}^{(\tau)}, u \in \mathcal{S}_{T}\left(\mathbb{R}^{n}\right)
$$

for any $i$.

We denote by $A_{s, k}\left(s \in \boldsymbol{R}, k \in \boldsymbol{Z}_{+}\right)$the set of functions $R(x, \xi)$ in $C^{\infty}\left(\boldsymbol{R}^{n} \times \boldsymbol{R}^{n}\right)$ of the form

$$
R(x, \xi)=a_{0}\left(x, \xi^{\prime}\right) \xi_{1}^{k}+\cdots+a_{k}\left(x, \xi^{\prime}\right), a_{i} \in S_{1,0}^{s+k+i}\left(\boldsymbol{R}^{n} \times \boldsymbol{R}^{n-1}\right) .
$$

Then if $R_{i} \in A_{s_{i}, k_{i}}(i=1,2)$, we have that $R_{1} \circ R_{2}, R_{1} R_{2} \in A_{s_{1}+s_{2}, k_{1}+k_{2}}$, and if $R \in A_{s, k}$ and $\alpha, \beta \in \mathbb{Z}_{+}^{n}$ with $\alpha_{1} \leq k$, we have that $R_{(\beta)}^{(\alpha)} \in A_{s-|\alpha|, k-\alpha_{1}}$.

Using partial fraction decomposition we see that if $|\alpha|+j=m$ and $j \leq m$ -1 we can write

$$
\xi^{\prime \alpha} \xi_{1}^{j}=\sum_{k=1}^{m} R_{k}(x, \xi) \prod_{i \neq k} \tilde{Q}_{i}(x, \xi)
$$

where $R_{k} \in A_{l_{k}, l_{k}-1}$.

Next using that for $|\alpha|=1$

$$
\left\langle\xi^{\prime}\right\rangle^{-1 / 2} \circ\left(\widetilde{P}_{m}\right)_{(\alpha)}-\sum_{k=1}^{m}\left\langle\xi^{\prime}\right\rangle^{-1 / 2} \circ\left(\tilde{Q}_{k}\right)_{(\alpha)} \circ\left[\prod_{i \mp k} \tilde{Q}_{i}\right] \in A_{m-(3 / 2), m-2}
$$

and (9.8) we get that for large $\tau T^{2}$ and $T^{-1}$ and $u \in \mathcal{S}_{T / 2}\left(\boldsymbol{R}^{n}\right)$

$$
\begin{aligned}
& T^{-1 / 2}\left\|E_{-1 / 2}\left(\widetilde{P}_{m}\right)_{(\alpha)}(x, D) u\right\|_{T}^{(\tau)} \leq C_{1}\left(\sum_{k=1}^{m}\left\|\left[\tilde{Q}_{k} \circ \prod_{i \neq k} \tilde{Q}_{i}\right](x, D) u\right\|_{T}^{(\tau)}+T\|u\|_{m-1, T}^{(\tau)}\right) \\
& \quad \leq C_{2}\left(\left\|\widetilde{P}_{m}(x, D) u\right\|_{T}^{(\tau)}+\left(\left(\tau T^{2}\right)^{1 / 2}+T\right)\|u\|_{m-1, T}^{(\tau)}\right) \\
& \quad \leq C_{3}\left\|\widetilde{P}_{m}(x, D) u\right\|_{T}^{(\tau)} .
\end{aligned}
$$

The inequality for $\left(\widetilde{P}_{m}\right)^{(\alpha)}$ can be deduced similarly. This completes the proof in case (i).

Next, we consider case (ii). We note that $C\left|\operatorname{Im} \tilde{\lambda}_{i}\right| \geq 1+\left|\xi^{\prime}\right|$ and $\left|\operatorname{Im} \tilde{\lambda}_{i}\right|$ $\geq 2\left|\tilde{c}_{i}\right|$. In case (ii) we shall use Proposition 1.1.

If $m_{1}+m_{2}-r=1$, if follows that $m=3$. Thus the desired estimate is nothing but Proposition 1.1. So we may assume that $m_{1}+m_{2}-r \geq 2$.

We set $\tilde{Q}_{i}=\tilde{p}_{i}+\tilde{q}_{i}$ and $Q^{(k)}=\prod_{i \neq k} \tilde{Q}_{i}$. Since for any $\left(x, \xi^{\prime}\right)$ equations $\tilde{p}_{i}(x, \xi)=0$ in $\xi_{1}\left(i=1, \cdots, m_{1}+m_{2}-r\right)$ have no common root, from partial fraction decomposition we have that if $|\alpha|+j=m$ and $j \leq m-1$

$$
\xi^{\prime \alpha} \xi_{1}^{j}-\sum_{k=1}^{m_{1}+m_{2}-r} R_{k} \circ Q^{(k)} \in A_{m-1, m-1}
$$


for some $R_{k} \in A_{l_{k}, l_{k}-1}$. Then from Proposition 1.1, we have that for large $\tau T^{2}$ and $T^{-1}$, and $u \in \mathcal{S}_{T / 2}\left(\mathbb{R}^{n}\right)$

$$
\begin{aligned}
& \tau^{-3 / 2} T^{-3} \sum_{0<|\alpha| \leq m}\left\|D^{\prime \alpha} D_{1}^{m-|\alpha|} u\right\|_{T}^{(\tau)} \\
& \quad \leq C\left(\sum_{k=1}^{m_{1}+m_{2}-r}\left\|Q_{k} \circ Q^{(k)}(x, D) u\right\|_{T}^{(\tau)}+(\tau T)^{-1}\|u\|_{m-1, T}^{(\tau)}\right) .
\end{aligned}
$$

On the other hand as in the proof of Lemma 4.3 we see that for $u \in \mathcal{S}\left(\boldsymbol{R}^{n}\right)$

$$
\left\|D_{1}^{m} u\right\|_{T}^{(\tau)} \leq\|Q(x, D) u\|_{T}^{(\tau)}+C\left((\tau T)^{-1}\left\|D_{1}^{m} u\right\|_{T}^{(\tau)}+\sum_{\alpha \neq 0}\left\|D^{\prime \alpha} D_{1}^{m-|\alpha|} u\right\|_{T}^{(\tau)}\right) .
$$

This inequality, (9.9), and that $\|u\|_{m, T}^{(\tau)} \leq C\left(\tau T^{2}\right)^{-3 / 2} \sum_{|\alpha| \leq m}\left\|D^{\prime \alpha} D_{1}^{m-|\alpha|} u\right\|_{T}^{(\tau)}$ for $u \in S_{T / 2}\left(\mathbb{R}^{n}\right)$ which follows from an interpolation on Sobolev norms on $x^{\prime}$ and the first inequality in the proof of Lemma 4.3 imply that if $\tau T^{2}$ and $T^{-1}$ are large

$$
\|u\|_{m, T}^{(\tau)} \leq C\left(\left(\tau T^{2}\right)^{-3 / 2}\|Q(x, D) u\|_{T}^{(\tau)}+\sum_{k=1}^{m_{1}+m_{2}-r}\left\|\tilde{Q}_{k} \circ Q^{(k)}(x, D) u\right\|_{T}^{(\tau)}\right)
$$

for $u \in \mathcal{S}_{T / 2}\left(\mathbb{R}^{n}\right)$.

We have to estimate the summation on the right in (9.10). We set $Q_{i k}=$ $\prod_{j \neq i, k} \tilde{Q}_{j}$. Since

$$
\tilde{Q}_{k} \circ Q^{(k)}-Q+\sqrt{-1} \sum_{|\alpha|=1} \sum_{i=k}\left\langle\xi^{\prime}\right\rangle^{1 / 2} \circ\left(\tilde{Q}_{k}\right)^{(\alpha)} \circ\left\langle\xi^{\prime}\right\rangle^{-1 / 2} \circ\left(\tilde{Q}_{i}\right)_{(\alpha)} \circ Q_{i k} \in A_{m-2, m-2}
$$

we have from Proposition 1.1 that for large $\tau T^{2}$ and $T^{-1}$, and $u \in \mathcal{S}_{T / 2}\left(\mathbb{R}^{n}\right)$

$$
\begin{aligned}
& \left\|\tilde{Q}_{k} \circ Q^{(k)}(x, D) u\right\|_{T}^{(\tau)} \leq\|Q(x, D) u\|_{T}^{(\tau)} \\
& \quad+C\left(\sum_{|\alpha|=1} \sum_{i \neq k} T^{1 / 2}\left\|\tilde{Q}_{k}(x, D) E_{-1 / 2}\left[\left(\tilde{Q}_{i}\right)_{(\alpha)} \circ Q_{i k}\right](x, D) u\right\|_{T}^{(\tau)}\right. \\
& \left.\quad+\sum_{j \leq m-2}\left\|E_{j} D_{1}^{m-2-j} u\right\|_{T}^{(\tau)}\right) .
\end{aligned}
$$

Setting $Q_{i \alpha}=\left\langle\xi^{\prime}\right\rangle^{-1 / 2} \circ\left(\tilde{Q}_{\dot{\xi}}\right)_{(\alpha)}$ we have that $\left(\tilde{Q}_{k} \circ Q_{i \alpha}-Q_{i \omega^{\circ}} \circ \tilde{Q}_{k}\right) \circ Q_{i k}$ and $Q_{i \alpha^{\circ}}$ $\tilde{Q}_{k} \circ Q_{i k}-Q_{i \alpha} \circ Q^{(i)}$ are in $A_{m-(3 / 2), m-2}$. Thus from Proposition 1.1 a term in the first summation on the right of (9.11) is dominated by a constant multiple of $T\left\|\tilde{Q}_{\dot{\delta}} \circ Q^{(i)}(x, D) u\right\|_{T}^{(\tau)}+\sum_{j \leq m-2}\left\|E_{m-(3 / 2)-j} D_{1}^{j} u\right\|_{T}^{(\tau)}$ if $\tau T^{2}$ and $T^{-1}$ are large and $u \in \mathcal{S}_{T / 2}\left(\mathbb{R}^{n}\right)$. Thus applying this estimate to (9.11) and summing up it in $k$ we obtain that for large $\tau T^{2}$ and $T^{-1}$

$$
\|u\|_{m, T}^{(\tau)}+\sum_{k=1}^{m_{1}+m_{2}-r}\left\|\tilde{Q}_{k} \circ Q^{(k)}(x, D) u\right\|_{T}^{(\tau)} \leq C\|Q(x, D) u\|_{T}^{(\tau)}, u \in \mathcal{S}_{T / 2}\left(\mathbb{R}^{n}\right)
$$

Finally using $\left\langle\xi^{\prime}\right\rangle^{-1 / 2} \circ Q_{(\alpha)}-\left\langle\xi^{\prime}\right\rangle^{-1 / 2} \circ\left[\sum_{k=1}^{m_{1}+m+2-r}\left(\tilde{Q}_{k}\right)_{(\omega)} \circ Q^{(k)}\right] \in A_{m-(3 / 2), m-2}$ 
we have from Proposition 1.1 that for large $\tau T^{2}$ and $T^{-1}$, and $u \in \mathcal{S}_{T / 2}\left(\boldsymbol{R}^{n}\right)$

$$
\left\|E_{-1 / 2} Q_{(\alpha)}(x, D) u\right\|_{T}^{(\tau)} \leq C\left(\sum_{k=1}^{m_{1}+m_{2}-r} T^{1 / 2}\left\|\tilde{Q}_{k} \circ Q^{(k)}(x, D) u\right\|_{T}^{(\tau)}+T^{3 / 2}\|u\|_{m-1, T}^{(\tau)}\right) .
$$

Similarly $\left\|E_{1 / 2} Q^{(\alpha)}(x, D) u\right\|_{T}^{(\tau)}$ is dominated by the same expression as the right of the above inequality. Combining (9.12) and these estimate we obtain the desired inequlity. This completes the proof in case (ii) and therefore the proof of Lemma 9.2.

Now we can complete the proof of Lemma 9.1 by patching the estimates in Lemma 9.2. We assume the notation $A_{s, k}$ in the proof of Lemma 9.2. Choose $C^{\infty}$-functions $\chi_{j}\left(\xi^{\prime}\right)$ on $\mathbb{R}^{n-1}(j=1, \cdots, s)$ which is homogeneous degree 0 for $\left|\xi^{\prime}\right|>1$ and satisfy $\sum_{j=1}^{s} \chi_{j}\left(\xi^{\prime}\right)=1$ for $\left|\xi^{\prime}\right|>1$ so that there exists an open neighbourhood $V$ of the origin in $\mathbb{R}^{n}$ such that for any $j \in\{1, \cdots, s\}$ there exists $R_{j} \in A_{m, m}$ with $R_{j}-\xi_{1}^{m} \in A_{m, m-1}$ such that $P(x, \xi)=R_{j}(x, \xi)$ on $V \times \operatorname{supp} \chi_{j}$ and the inequality in case (ii) of Lemma 9.2 holds with $R_{j}$ for $Q$.

Let us choose $\delta_{0}>0$ with $\overline{B_{2 \delta_{0}}(0)} \subset V$. Let $\phi \in C^{\infty}\left(\mathbb{R}^{n}\right)$ with $\phi(t)=1$ when $|t| \leq 1, \phi(t)=0$ when $|t| \geq \frac{3}{2}$, and set $\widetilde{P}(x, \xi)=P\left(\phi\left(\delta_{0}^{-1}|x|\right) x, \xi\right)$. Let $\chi_{0} \in$ $C_{0}^{\infty}\left(B_{\delta_{0}}(0)\right)$ with $\chi_{0}=1$ on $\overline{B_{\delta_{0} / 2}(0)}$. We set $\varphi_{j}\left(x, \xi^{\prime}\right)=\chi_{0}(x) \chi_{j}\left(\xi^{\prime}\right)(j=1, \cdots, s)$, $\varphi_{0}\left(x, \xi^{\prime}\right)=\chi_{0}(x)\left(1-\sum_{j=1}^{s} \chi_{j}\left(\xi^{\prime}\right)\right)+\left(1-\chi_{0}(x)\right)$.

Then we have that $\sum_{j=0}^{s} \varphi_{j}\left(x, \xi^{\prime}\right)=1, \widetilde{P}(x, \xi)=R_{j}(x, \xi)$ for $\left(x, \xi^{\prime}\right) \in \operatorname{supp} \varphi_{j}$ $(j=1, \cdots, s)$.

Thus we have for large $\tau T^{2}$ and $T^{-1}$, and $u \in \mathcal{S}_{T / 2}\left(\boldsymbol{R}^{n}\right)$ that

$$
\|u\|_{m, T}^{(\tau)} \leq \sum_{j=0}^{s}\left\|\varphi_{j}\left(x, D^{\prime}\right) u\right\|_{m, T}^{(\tau)} \leq\left\|\varphi_{0}\left(x, D^{\prime}\right) u\right\|_{m, T}^{(\tau)}+C \sum_{j=1}^{s}\left\|\left(R_{j} \circ \varphi_{j}\right)(x, D) u\right\|_{m, T}^{(\tau)} .
$$

Since $R_{j} \circ \varphi_{j}-\varphi_{j} \circ \widetilde{P}-\sqrt{-1} \sum_{|\alpha|=1}\left(\left\langle\xi^{\prime}\right\rangle^{-1 / 2} \circ R_{j(\alpha)} \circ\left[\varphi_{j}^{(\alpha)}\left\langle\xi^{\prime}\right\rangle^{1 / 2}\right]-\left\langle\xi^{\prime}\right\rangle^{1 / 2} \circ R_{j}^{(\alpha)} \circ\right.$ $\left.\left[\varphi_{j(\alpha)}\left\langle\xi^{\prime}\right\rangle^{-1 / 2}\right]\right)$ is in $A_{m-2, m-1}$, we have that for any $\tau, T, u \in \mathcal{S}_{T}\left(\mathbb{R}^{n}\right)$

$$
\begin{aligned}
\|\left(R_{j} \circ \varphi_{j}\right) & (x, D) u \|_{T}^{(\tau)} \\
\leq & C\left(\|\widetilde{P}(x, D) u\|_{T}^{(\tau)}+T^{1 / 2} \sum_{|\alpha|=1} \|\left[R_{j} \circ\left(\varphi_{j}^{(\alpha)}\left\langle\xi^{\prime}\right\rangle^{1 / 2}\right](x, D) u \|_{T}^{(\tau)}\right.\right. \\
& +T^{1 / 2} \sum_{|\alpha|=1}\left\|\left[R_{j} \circ\left(\varphi_{j(\alpha)}\left\langle\xi^{\prime}\right\rangle^{-1 / 2}\right)\right](x, D) u\right\|_{T}^{(\tau)} \\
& \left.+\tau^{-1 / 2} T\|u\|_{m-2, T}^{(\tau)}+\left\|E_{-1} D_{1}^{m-1} u\right\|_{T}^{(\tau)}\right) .
\end{aligned}
$$

We set $\tilde{\varphi}_{i \alpha}=\varphi_{j}^{(\alpha)}\left\langle\xi^{\prime}\right\rangle^{1 / 2}, \tilde{\tilde{\varphi}}_{j(1)}=\left(\varphi_{j}\right)_{(\alpha)}\left\langle\xi^{\prime}\right\rangle^{-1 / 2}$. Then we have that $R_{j} \circ \tilde{\varphi}_{j \alpha}-$ $\widetilde{\varphi}_{j \omega} \circ \widetilde{P}$ and $R_{j} \circ \tilde{\tilde{\varphi}}_{j \alpha}-\tilde{\tilde{\varphi}}_{j \alpha} \circ \widetilde{P}$ are in $A_{m-(3 / 2), m-1}$. Thus we have from the above equality that for large $\tau T^{2}$ and $T^{-1}$, and $u \in \mathcal{S}_{T / 2}\left(\mathbb{R}^{n}\right)$ 


$$
\begin{aligned}
& \left\|\left(R_{j} \circ \varphi_{j}\right)(x, D) u\right\|_{T}^{(\tau)} \\
& \quad \leq C\left(\|\widetilde{P}(x, D) u\|_{T}^{(\tau)}+T^{2}\|u\|_{m-1, T}^{(\tau)}+\left\|E_{-1 / 2} D_{1}^{m-1} u\right\|_{T}^{(\tau)}\right) .
\end{aligned}
$$

As in the proof of Lemma 4.3 the last term on the right of the above inequality can be dominated by $C^{\prime}\left\{(\tau T)^{-1}\|\widetilde{P}(x, D) u\|_{T}^{(\tau)}+T^{3 / 2}\|u\|_{m, T}^{(\tau)}\right\}$ for any $\tau$, $T, u \in \mathcal{S}_{T / 2}\left(\mathbb{R}^{n}\right)$. Thus we get from $(9.13)$ and the above inequality that for large $\tau T^{2}$ and $T^{-1}$, and $u \in S_{T / 2}\left(\mathbb{R}^{n}\right)$

$$
\|u\|_{m, T}^{(\tau)} \leq\left\|\varphi_{0}\left(x, D^{\prime}\right) u\right\|_{m, T}^{(\tau)}+C\|\widetilde{P}(x, D) u\|_{T}^{(\tau)} .
$$

Finally using Leibniz rule we see that for any $\tau, T, u \in \mathcal{S}_{T}\left(\mathbb{R}^{n}\right) \cap C_{0}^{\infty}\left(B_{\delta_{0} / 8}(0)\right)$

$$
\left\|\varphi_{0}\left(x, D^{\prime}\right) u\right\|_{m, T}^{(\tau)} \leq C\left(\tau^{-1 / 2} T\|u\|_{m-2, T}^{(\tau)}+\left\|E_{-1} D_{1}^{m-1} u\right\|_{T}^{(\tau)}+\left\|E_{-2} D_{1}^{m} u\right\|_{T}^{(\tau)}\right)
$$

because $\varphi_{0}\left(x, D^{\prime}\right) u=\varphi_{0}\left(x, D^{\prime}\right)\left(\chi_{1} u\right)$ for $u \in C_{0}^{\infty}\left(B_{\delta_{0} / 8}(0)\right)$ and $\varphi_{0} \circ \chi_{1} \in S^{-\infty}$ with a notation that $\chi_{1}(x)=\chi_{0}(4 x)$. Again, the latter two terms on the right of the above inequality can be dominated by $C^{\prime}\left\{\left(1+(\tau T)^{-1}\right)\|\widetilde{P}(x, D) u\|_{T}^{(\tau)}+\tau^{-1 / 2} T\right.$ $\left.\|u\|_{m-1, T}^{(\tau)}\right\}$ for any $\tau, T, u \in \mathcal{S}_{T / 2}\left(\mathbb{R}^{n}\right)$. Thus we have that for any $\tau, T, u \in \mathcal{S}_{T / 2}$ $\left(\mathbb{R}^{n}\right) \cap C_{0}^{\infty}\left(B_{\delta_{0} / 8}(0)\right)$

$$
\left\|\varphi_{0}\left(x, D^{\prime}\right) u\right\|_{m, T}^{(\tau)} \leq C\left\{\left(1+(\tau T)^{-1}\right)\|\widetilde{P}(x, D) u\|_{T}^{(\tau)}+\tau^{-1 / 2} T\|u\|_{m-1, T}^{(\tau)}\right\} .
$$

Substituting this inequality into (9.14) we get the desired result with $\frac{\delta_{0}}{8}$ for $\delta_{0}$ in the lemma because $\widetilde{P}(x, D) u=P(x, D) u$ for $u \in C_{0}^{\infty}\left(B_{\delta_{0}}(0)\right)$. The proof is complete.

\section{References}

[1] Beal., R. and Fefferman, C., Spatially inhomogeneous pseudo-differential operators I, Comm. Pure Appl. Math., 27 (1974), 1-24.

[2] Calderón, A.P., Uniqueness in the Cauchy problem for partial differential equations, Amer. J. Math., 80 (1958), 16-36.

[3] Fujii, S., On the uniqueness of solution to the Cauchy problem for elliptic equations in two variables, To appear.

[4] Hörmander, L., On the uniqueness of the Cauchy problem I, II, Math. Scand., 6 (1958), 213-225, 7 (1959), 177-190.

[5] Mizohata S., Unicité du pro'ongement des solutions des équations du quatrième ordere, Proc. Japan Acad., 34 (1958), 687-692.

[6] Watababe, K., On the uniqueness of the Cauchy problem for certain elliptic equation with triple characteristics, Tohoku Math. Journ., 23 (1971), 473-490.

[7] Watanabe, K., and Zuily, C., On the uniqueness of the Cauchy problem for elliptic differenial operators with smooth characteristics of variable multiplicity, Comm. P. D.E., 2(8) (1977), 831-855.

[8] Zuily, C., Uniqueness and non-uniqueness in the Cauchy problem, Progress in Math. 33, Birkhäuser. 